\title{
Existence and uniqueness of Rayleigh waves in isotropic elastic Cosserat materials and algorithmic aspects
}

\author{
Hassam Khan ${ }^{1}$ and Ionel-Dumitrel Ghiba ${ }^{2}$ and \\ Angela $\mathrm{Madeo}^{3}$ and Patrizio Neff ${ }^{4}$
}

February 2, 2022

\begin{abstract}
We discuss the propagation of surface waves in an isotropic half space modelled with the linear Cosserat theory of isotropic elastic materials. To this aim we use a method based on the algebraic analysis of the surface impedance matrix and on the algebraic Riccati equation, and which is independent of the common Stroh formalism. Due to this method, a new algorithm which determines the amplitudes and the wave speed in the theory of isotropic elastic Cosserat materials is described. Moreover, the method allows us to prove the existence and uniqueness of a subsonic solution of the secular equation, a problem which remains unsolved in almost all generalised linear theories of elastic materials. Since the results are suitable to be used for numerical implementations, we propose two numerical algorithms which are viable for any elastic material. Explicit numerical calculations are made for alumunium-epoxy in the context of the Cosserat model. Since the novel form of the secular equation for isotropic elastic material has not been explicitly derived elsewhere, we establish it in this paper, too.
\end{abstract}

Keywords: Cosserat elastic materials, Matrix analysis method, Riccati equation, Rayleigh waves, Stroh formalism, secular equation, existence and uniqueness.

AMS 2020 MSC: 74J15, 74M25, 74H05, 74J05

\section{Contents}

1 Introduction

2 Statement of the problem

2.1 Notation . . . . . . . . . . . . . . 6 6

2.2 Cosserat theory of isotropic elastic solids as particular case of the relaxed micromorphic model $\ldots \ldots \ldots$

2.3 Real plane waves in isotropic Cosserat elastic solids . . . . . . . . . . . . . . . . . . . . . . . . . . . . 10

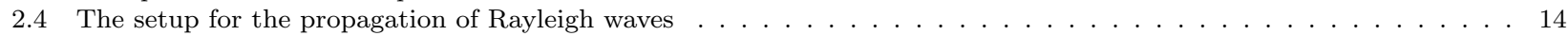

3 The ansatz for the solution and the limiting speed

4 The common method to construct the solution using the Stroh formalism

5 The new secular equation. Existence and uniqueness of Rayleigh waves

5.1 Derivation and matrix analysis of the algebraic Riccati equation . . . . . . . . . . . . . . . . 25

5.2 The main result: Existence and uniqueness of Rayleigh waves . . . . . . . . . . . . . . . . . . . . . . . . . . . . . . .

${ }^{1}$ Hassam Khan, Lehrstuhl für Nichtlineare Analysis und Modellierung, Fakultät für Mathematik, Universität Duisburg-Essen, Thea-Leymann Str. 9, 45127 Essen, Germany, email: hassam.khan@stud.uni-due.de

${ }^{2}$ Ionel-Dumitrel Ghiba (Corresponding author), Department of Mathematics, Alexandru Ioan Cuza University of Iaşi, Blvd. Carol I, no. 11, 700506 Iaşi, Romania; and Octav Mayer Institute of Mathematics of the Romanian Academy, Iaşi Branch, 700505 Iaşi, email: dumitrel.ghiba@uaic.ro

${ }^{3}$ Angela Madeo, Technische Universität Dortmund, August-Schmidt-Str. 8,44227 Dortmund, Germany, email: angela.madeo@tu-dortmund.de

${ }^{4}$ Patrizio Neff, Head of Lehrstuhl für Nichtlineare Analysis und Modellierung, Fakultät für Mathematik, Universität DuisburgEssen, Thea-Leymann Str. 9, 45127 Essen, Germany, email: patrizio.neff@uni-due.de 
7 From linear Cosserat theory to classical linear elasticity: A consistency check and comparison of the results 34

\section{Introduction}

Traveling waves can exist within a small depth from a free surface of an elastic continuum, while the bulk of the continuum remains almost at rest. Such waves are called Rayleigh waves named after the English physicist Lord Rayleigh, who carried out pioneering work in studies of wave propagations in isotropic elastic media [60]. Rayleigh waves are important for modelling seismic waves that are created by earthquakes on the surface of the earth. These waves are a combination of longitudinal (horizontal) and transverse (vertical) motions. The horizontal components of the displacement are parallel to the direction of propagation, whereas vertical components are directed into the half-space. Rayleigh waves move elliptically, they take place counter-clockwise near the surface and clockwise deep down. The amplitude of the waves decays exponentially with depth beneath the surface. The studies of Rayleigh waves captivated the attention of many scientists owing to its industrial application such as material characterization, nondestructive evaluation and acoustic microscopy. These waves are also employed to detect cracks and other defects in the material. In addition, many applications have been found in seismology and near surface geophysical exploration.

Rayleigh waves are particular inhomogeneous plane waves. Inhomogeneous plane waves, also known as evanescent waves, represent those waves for which the planes of constant phase are not the same as the planes of constant amplitude. Usually, these types of waves are described in terms of the slowness bivector (a complex vector) and the amplitude bivector. Hayes [29, 8] has developed the directional-ellipse method for a systematic study of all inhomogeneous plane waves that may propagate in classical linear elasticity. In classical elasticity of isotropic materials, the solution of the corresponding equation for Rayleigh surface waves speed (the secular equation) has been studied numerically, see e.g. [60,30]. For the statement of the problem one may consult the book [1] and the works [55, 56, 59, 46, 41, 16, 69, 70, 71, 72, 73].

An important non-trivial task in classical linear elasticity is the extension of the results concerning the Rayleigh waves to anisotropic materials. Using a formalism (Stroh formalism) constructed in [63], Stroh [64] was able to avoid the complex secular equation derived by Synge [65] and he has given a real expression of it, see also Currie [14]. Another issue concerning the surface wave in an anisotropic elastic half-space is the derivation of the explicit secular equations such that a solution (analytical, if possible) can be easily found (i.e. at least with the help of a clear numerical strategy). In this respect, we mention [66, 50, 15, 16, 18, 68, 68]. For isotropic as well as for anisotropic materials, the mathematical analysis of this equation has, perhaps, at least the same importance as the derivation of the secular equations, since it is not obvious if there exists an admissible solution of the secular equation and if this solution is unique. By an admissible solution we mean a solution which takes into account all the restrictions which were imposed in the derivation of the secular equation. In many approaches this essential aspect is neglected or only conjectured. For linear isotropic materials this is explained in the book by Achenbach [1]. While strong ellipticity (Legendre-Hadamard ellipticity) guarantees the existence of plane waves, it is not obvious if seismic waves exist since three inhomogeneous body wave solutions are needed when the traction-free boundary condition specific to seismic waves is solved. For seismic waves propagating in anisotropic elastic materials, the first uniqueness result is due to Barnett et al. [6], see also [9] for more details, while for the proof of the existence of the solution of the secular equation, we mention the works by Barnett and Lothe [4], Lothe and Barnett [42] and Ingebrigtsen and Tonning [31].

Motivated by previous work of Mielke and Sprenger [48] which is more related to control theory [33] than to surface wave propagation, Fu and Mielke [24] and Mielke and Fu [47] have devised a new method for anisotropic elastic materials which is not based on the Stroh formalism, it is conceptually different from the other methods and it is mathematically well explained. They have shown that the impedance matrix [31] defining the secular equation is the solution of an algebraic Riccati equation. Using the properties of this equation, in [47, 24] it is then proven that the secular equation does not admit spurious roots. It can be said that the works by Mielke and $\mathrm{Fu}$ give an elegant final answer to the general case of anisotropic elastic materials, in the framework of classical linear elasticity. Since in $[47,24]$ the secular equation is not written explicitly, in the current paper we 
have computed it for linear isotropic classical elasticity, too, and we compare it with the classical one and that resulting by using the Stroh formalism.

However, the classical theory of elasticity does not explain certain discrepancies that occur in propagation of waves at high frequency and short wavelength. It is now a common place that the response of the material to external stimuli depends heavily on the motions of its inner structure and if the ratio of the characteristic length associated with the external stimuli and the internal characteristic length is near to 1 , then the response of constituent subcontinua becomes important, see [20]. Classical linear elasticity ignores these effects.

One of the first generalization of classical linear elasticity is represented by the Cosserat (micropolar) continuum theory in which the rotational degrees of freedom play a central role [22, 49]. For a quick comparison, we mention that denoting the macroscopic displacement by $\mathbf{u}: \Omega \subset \mathbb{R}^{3} \mapsto \mathbb{R}^{3}$ and the microrotation vector field by $\vartheta: \Omega \subset \mathbb{R}^{3} \mapsto \mathbb{R}^{3}$, the elastic energy density of the isotropic linear Cosserat model read

$$
\begin{aligned}
W(\mathrm{D} u, \vartheta, \mathrm{D} \vartheta)= & \mu_{\mathrm{e}}\left\|\operatorname{dev}_{3} \operatorname{sym} \mathrm{D} u\right\|^{2}+\mu_{\mathrm{c}} \| \text { skew }(\mathrm{D} u-\operatorname{Anti} \vartheta) \|^{2}+\frac{2 \mu_{\mathrm{e}}+3 \lambda_{\mathrm{e}}}{6}[\operatorname{tr}(\mathrm{D} u)]^{2} \\
& +\frac{\mu_{\mathrm{e}} L_{\mathrm{c}}^{2}}{2}\left[\alpha_{1}\left\|\operatorname{dev}_{3} \operatorname{sym} \mathrm{D} \vartheta\right\|^{2}+\alpha_{2} \| \text { skew } \mathrm{D} \vartheta \|^{2}+\frac{2 \alpha_{1}+3 \alpha_{3}}{6}[\operatorname{tr}(\mathrm{D} \vartheta)]^{2}\right],
\end{aligned}
$$

where $\left(\lambda_{\mathrm{e}}, \mu_{\mathrm{e}}\right), \mu_{\mathrm{c}}, L_{\mathrm{c}}, \alpha_{1}, \alpha_{2}$ and $\alpha_{3}$ are six isotropic elastic moduli representing the parameters related to the meso-scale, the Cosserat couple modulus, the characteristic length, and the three general isotropic curvature parameters, respectively, $(\operatorname{Anti}(\vartheta))_{i j}=-\epsilon_{i j k} \vartheta_{k}$, with $\epsilon_{i j k}$ the totally antisymmetric third order permutation tensor, see Section 2.1, while the elastic energy density of classical linear elasticity is

$$
W(\mathrm{D} u)=\mu_{\mathrm{e}}\left\|\operatorname{dev}_{3} \operatorname{sym} \mathrm{D} u\right\|^{2}+\frac{2 \mu_{\mathrm{e}}+3 \lambda_{\mathrm{e}}}{6}[\operatorname{tr}(\mathrm{D} u)]^{2} .
$$

In the framework of the Cosserat theory, the propagation of seismic waves in an isotropic Cosserat elastic half space was studied in [11] using the Stroh formalism [16, 64], see also [40]. The strong point of the approach in [11] is that explicit expressions of involved eigenvalue problems and explicit conditions upon the wave speed were found, as well as the exact expressions of three linear independent amplitude vectors. Then, a simple form of the secular equation is obtained, which, by comparison with other generalized forms of the secular equation for Cosserat materials $[20,23,27,34,35,36,37,38]$ does not involve the complex form of the attenuating coefficients. For a specified class of materials, i.e., for which the constitutive coefficients ${ }^{1}$ satisfy

$$
\mu_{\mathrm{e}}-\mu_{\mathrm{c}}+\lambda_{\mathrm{e}}>0, \quad \mu_{\mathrm{e}}+\mu_{\mathrm{c}}>0, \quad \alpha_{1}+\alpha_{2}>0,
$$

Chiriţa and Ghiba [11, Eq. (4.14)] have proved the existence of the solution of their secular equation. Using some illustrative graphics they conjectured that the solution should be also unique for this subclass of materials, but there does not exist a proof of the uniqueness in the Cosserat theory or an existence and uniqueness proof for a larger class of isotropic Cosserat materials.

It is also important to notice that in the Cosserat theory, too, it is not obvious how to avoid the spurious roots of the secular equation, as long as the Stroh formalism is used. Even if the relation between Mielke and Fu's method $[47,24]$ and the Cosserat model is not evident, in our paper we show that it may be adapted to the study of propagation of seismic waves in Cosserat materials. So, our derivation will involve a matrix algebraic Riccati equation which will provide a formula for the desired solution. We will show that the new form of the secular equations, written in terms of the impedance matrix, does not admit any spurious root, i.e. there exists only one subsonic surface wave.

In the current paper we prove both the existence and the uniqueness of the wave speed of the Rayleigh wave in Cosserat materials, for the first time, and this under weaker conditions ${ }^{2}$ on the constitutive coefficients, i.e., we require only

$$
2 \mu_{\mathrm{e}}+\lambda_{\mathrm{e}}>0, \quad \mu_{\mathrm{e}}>0, \quad \mu_{\mathrm{c}}>0, \quad \alpha_{1}+\alpha_{2}>0 .
$$

For a given direction $\xi \in \mathbb{R}^{3}, \xi \neq 0$ of the form $\xi=\left(\xi_{1}, \xi_{2}, 0\right)^{T}$, the constitutive requirements (1.4) are equivalent to the existence of only real waves propagating in the plane normal to the direction of propagation and parallel

\footnotetext{
${ }^{1}$ We use different notations in comparison to the Eringen notation in [11], i.e. $\mu_{\mathrm{c}}=\frac{\kappa_{\text {Eringen }}}{2}, \mu_{\mathrm{e}}=\mu_{\text {Eringen }}+\frac{\kappa_{\text {Eringen }}}{2}$, see also [28]. In the Eringen notations these are $\lambda_{\text {Eringen }}+\mu_{\text {Eringen }}>0, \kappa_{\text {Eringen }}>0, \alpha_{1}+\alpha_{2}>0$.

${ }^{2}$ In the sense that the conditions $\mu_{\mathrm{e}}-\mu_{\mathrm{c}}+\lambda_{\mathrm{e}}>0, \mu_{\mathrm{e}}+\mu_{\mathrm{c}}>0$ imply $2 \mu_{\mathrm{e}}+\lambda_{\mathrm{e}}>0$ but not vice versa, even when the extra conditions $\mu_{\mathrm{c}}>0$ and $\mu_{\mathrm{e}}>0$ are also assumed.
} 
to the direction of propagation. These conditions do not involve the constitutive parameter $\alpha_{3}$ as it would be the case for the requirement that only real waves may propagate (in arbitrary planes) in any directions and for arbitrary wave numbers. Indeed, the propagation of real plane waves is equivalent to the constitutive inequalities

$$
2 \mu_{\mathrm{e}}+\lambda_{\mathrm{e}}>0, \quad \mu_{\mathrm{e}}>0, \quad \mu_{\mathrm{c}}>0, \quad \alpha_{1}+\alpha_{2}>0, \quad 2 \alpha_{1}+\alpha_{3}>0 .
$$

Let us notice that while in classical linear elasticity the existence of seismic waves is guaranteed for strongly elliptic materials, i.e.,

$$
2 \mu_{\mathrm{e}}+\lambda_{\mathrm{e}}>0, \quad \mu_{\mathrm{e}}>0
$$

in the Cosserat model the strong ellipticity conditions (Legendre-Hadamard ellipticity) [32, 61, 2] are equivalent to

$$
2 \mu_{\mathrm{e}}+\lambda_{\mathrm{e}}>0, \quad \quad \mu_{\mathrm{e}}+\mu_{\mathrm{c}}>0, \quad \alpha_{1}+\alpha_{2}>0, \quad 2 \alpha_{1}+\alpha_{3}>0
$$

and the latter conditions are not sufficient to impose the existence of seismic waves. The existence of seismic waves is rather related to the propagation of real plane waves than to strong ellipticity, while the strong ellipticity conditions are useful in the study of accelerated waves [2]. We mention that the existence for only real plane

\begin{tabular}{|c|c|c|}
\hline Name & Expression & $\begin{array}{l}\text { Dispersive waves/ } \\
\text { Non-dispersive waves }\end{array}$ \\
\hline $\begin{array}{l}\text { the velocity of the acoustic branch of transla- } \\
\text { tional compression (longitudinal) plane wave }\end{array}$ & $\mathfrak{c}_{p}=\sqrt{\frac{\lambda_{e}+2 \mu_{e}}{\rho}}$ & non-dispersive \\
\hline $\begin{array}{l}\text { the limit of the group/phase velocity of the } \\
\text { acoustic branch of the shear-rotational wave } \\
\text { at } \omega \rightarrow 0(k \rightarrow 0)\end{array}$ & $\mathfrak{c}_{t}=\sqrt{\frac{\mu_{e}}{\rho}}$ & dispersive \\
\hline $\begin{array}{l}\text { the limit of the group/phase velocity of the } \\
\text { acoustic branch of the shear-rotational wave } \\
\text { at } \omega \rightarrow \infty(k \rightarrow \infty)\end{array}$ & $\mathfrak{c}_{s}=\sqrt{\frac{\mu_{e}+\mu_{c}}{\rho}}$ & dispersive \\
\hline $\begin{array}{l}\text { the group/phase velocity for the compres- } \\
\text { sional rotational wave in the limit } \omega \rightarrow \infty \\
(k \rightarrow \infty)\end{array}$ & $\mathfrak{c}_{m, p}=\sqrt{\frac{L_{c}^{2}\left(2 \alpha_{1}+\alpha_{3}\right)}{\rho j \tau_{c}^{2}}}$ & dispersive \\
\hline $\begin{array}{l}\text { the limit of the group/phase velocity of the } \\
\text { acoustic branch of the shear-rotational wave } \\
\text { at } \omega \rightarrow \infty(k \rightarrow \infty)\end{array}$ & $\mathfrak{c}_{m, s}=\sqrt{\frac{L_{c}^{2}\left(\alpha_{1}+\alpha_{2}\right)}{\rho j \tau_{c}^{2}}}=\sqrt{\frac{L_{c}^{2} \gamma}{\rho j \tau_{c}^{2}}}$ & dispersive \\
\hline $\begin{array}{l}\text { the limit of the optical branch (compressional- } \\
\text { rotational and shear-rotational) at the cut-off } \\
\text { frequency } \omega=2 \sqrt{\frac{\mu_{c}}{\rho j \mu_{e} \tau_{c}^{2}}}, k=0\end{array}$ & $\begin{array}{l}0 \text { (group velocity }) \\
/ \infty(\text { phase velocity })\end{array}$ & dispersive \\
\hline
\end{tabular}
waves implies the strong ellipticity conditions but not vice versa. For the Cosserat theory, strong ellipticity is related only to the propagation of acceleration waves [19, 2].

Table 1: Some group velocities $c=\frac{\omega}{k}$ or/and phase velocities $\frac{d \omega}{d k}$ and the cut-off frequency in linear Cosserat elasticity.

Since different authors use different notation for elastic constants, we will also interpret almost all the conditions upon the constitutive parameters with the help of some relations between the velocities of compression/transversal acoustic/optical plane waves and with the help of some cut-off frequency of the optical 


\begin{tabular}{|l|c|c|}
\hline \multicolumn{1}{|c|}{ Name } & Expression & $\begin{array}{l}\text { Dispersive waves/ } \\
\text { Non-dispersive waves }\end{array}$ \\
\hline $\begin{array}{l}\text { the velocity of the acoustic branch of transla- } \\
\text { tional compression (longitudinal) plane wave }\end{array}$ & $c_{p}=\sqrt{\frac{\lambda_{e}+2 \mu_{e}}{\rho}}$ & non-dispersive \\
\hline $\begin{array}{l}\text { the limit of the group/phase velocity of the } \\
\text { acoustic branch of the shear-rotational wave } \\
\text { at } \omega \rightarrow 0(k \rightarrow 0)\end{array}$ & $c_{t}=\sqrt{\frac{\mu_{e}}{\rho}}$ & non-dispersive \\
\hline $\begin{array}{l}\text { the limit of the group/phase velocity of the } \\
\text { acoustic branch of the shear-rotational wave } \\
\text { at } \omega \rightarrow \infty(k \rightarrow \infty)\end{array}$ & $c_{t}=\sqrt{\frac{\mu_{e}}{\rho}}$ & non-dispersive \\
\hline $\begin{array}{l}\text { the group/phase velocity for the compres- } \\
\text { sional rotational wave in the limit } \omega \rightarrow \infty \\
(k \rightarrow \infty)\end{array}$ & $\times$ & not present \\
\hline $\begin{array}{l}\text { the limit of the group/phase velocity of the } \\
\text { acoustic branch of the shear-rotational wave } \\
\text { at } \omega \rightarrow \infty(k \rightarrow \infty)\end{array}$ & $\times$ & not present \\
\hline \begin{tabular}{l} 
cut-off frequency \\
\hline
\end{tabular} & $\times$ & not present \\
\hline
\end{tabular}

Table 2: The group velocities $c=\frac{\omega}{k}$ or/and phase velocities $\frac{d \omega}{d k}$ in classical linear elasticity.

branches and group velocities of plane bulk waves in the limit of high and low frequencies. This will give a better understanding of the physical sense of the formulae. To this aim, we will use the quantities from Table 1, see [20, Figure 5.11.2., Page 150] and [53]. In Table 2 we list the corresponding informations and notations for classical linear elasticity.

With the help of the quantities presented in Table 1, the first two inequalities of the set of conditions (1.3) considered by Chiriţă and Ghiba [11] imply that the translational compressional wave is real and that the shearrotational wave (optical branch) is real at high frequencies, the first inequality also implies that at the limit of high frequencies the translational compressional wave is faster than the shear-rotational wave (if they both exist), while the third one means that the shear-rotational wave (acoustic branch) is real at high frequencies. The inequalities (1.3) do not imply that the shear-rotational wave (optical branch) is real at low frequencies, i.e., $\omega \rightarrow 0$. In fact, the inequalities (1.3) do not imply that the plane waves are real, i.e., that the propagation plane waves are defined only by real frequencies.

To the contrary, the first implication of the set of conditions (1.5) means that all these waves (compressional/ shear-rotational waves, acoustic/optical branch) are real. Then, we can treat and interpret further the propagation of plane waves. It is clear that under conditions (1.5) all branches of waves are real for the entire range $[0, \infty)$ of the frequency. However, we may also see directly from the first condition $(1.5)_{1}$ that the translational compressional wave is real, the second condition $(1.5)_{2}$ means that the acoustic branch of the shear-rotational wave is real at low frequencies and together with the third condition $(1.5)_{3}$ means that the acoustic branch of the shear-rotational wave is real at high frequencies, the fourth inequality $(1.5)_{4}$ implies that the optical branch of the shear-rotational wave is real at high frequencies. In addition, the third inequality $(1.5)_{3}$ means that the optical branch of the shear-rotational wave at high frequencies has a larger velocity than the acoustic branch of the same wave at low frequencies (if they both exist, which is the case due to other conditions). We have just given the interpretation of the first four inequalities, but the fifth one expresses directly that the compressional rotational wave at high frequencies is real.

Considering the quantities from Tables 1 and 2, we may infer from (1.6) that in classical elasticity the strong ellipticity conditions correspond to the existence of real compressional and shear waves, while in the Cosserat case the corresponding conditions (1.7) (strong ellipticity conditions, Legendre-Hadamard ellipticity, 
the positive definiteness of the acoustic tensor) implies the existence of the real translational compressional wave on the entire range of real frequencies, of the real shear-rotational waves (both branches) at high frequencies, and of real rotational compressional waves at high frequencies, but at lower frequencies the latter waves may not be real since (1.7) does not guarantee that $\mathfrak{c}_{t}=\frac{\mu_{\mathrm{e}}}{\rho}$ is real. To the contrary, the conditions (1.5) imply that all these branches and types of plane wave are real, i.e., the group/phase velocities are real on the entire range of possible frequencies.

The structure of the present paper is now the following. In Section 2, after a short introduction of our notation, we present the linear Cosserat model for isotropic elastic materials as a special case of the relaxed micromorphic model. This comparison establishes the relations between these models and it is also useful for further studies, where the results obtained in the linear relaxed micromorphic model will be compared with those established in the Cosserat theory of linear elastic materials. Then, since a self contained study of the propagation of real waves in isotropic Cosserat elasticity is still missing, as well as the explicit form of the conditions on the constitutive coefficient which imply it, we dedicate Subsection 2.3 to it. In Subsection 2.4 we present the setup in the propagation of Rayleigh waves and we discuss the propagation of some special real plane wave which are related to the propagation of seismic waves. In Section 3 we give the ansatz of the solution and we define the limiting speed, by making the relation between the seismic waves and the special real plane waves considered in Subsection 2.4. In Section 4 we present the common method to construct the solution using the Stroh formalism and we put it in relation with our method, in order to conclude the section with some auxiliary results. In Section 5 we establish the new form of the secular equation using the novel method which allow to give the main result of the paper, i.e., the first proof in the literature of the existence and uniqueness of the subsonic speed of the secular equation, in the framework of isotropic linear Cosserat elastic models. In Section 6 we provide two numerical algorithms which can be implemented for any material once the constitutive coefficients are known. We present effective numerical results for an alumunium-epoxy composite. In Section 7 we provide the explicit form of the secular equations for isotropic linear elastic materials, since its explicit form has not been derived elsewhere and we compare it with other forms from the literature. We conclude the paper with some final remarks.

\section{Statement of the problem}

\subsection{Notation}

We consider that the mechanical behaviour of a body accupying the unbounded regular region of three dimensional Euclidean space is modelled with the help of the Cosserat theory of linear isotropic elastic materials. We denote by $n$ the outward unit normal on $\partial \Omega$. The body is referred to a fixed system of rectangular Cartesian axes $O x_{i}(i=1,2,3),\left\{e_{1}, e_{2}, e_{3}\right\}$ being the unit vectors of these axes.

In the following, we recall some useful notations for the present work. For $a, b \in \mathbb{R}^{3 \times 3}$ we let $\langle a, b\rangle_{\mathbb{R}^{3}}$ denote the scalar product on $\mathbb{R}^{3}$ with associated vector norm $\|a\|^{2}=\langle a, a\rangle$. We denote by $\mathbb{R}^{3 \times 3}$ the set of real $3 \times 3$ second order tensors, written with capital letters. Matrices will be denoted by bold symbols, e.g. $\mathbf{X} \in \mathbb{R}^{3 \times 3}$, while $X_{i j}$ will denote its component. The standard Euclidean product on $\mathbb{R}^{3 \times 3}$ is given by $\langle\mathbf{X}, \mathbf{Y}\rangle_{\mathbb{R}^{3 \times 3}}=$ $\operatorname{tr}\left(\mathbf{X} \mathbf{Y}^{T}\right)$, and thus, the Frobenious tensor norm is $\|\mathbf{X}\|^{2}=\langle\mathbf{X}, \mathbf{X}\rangle_{\mathbb{R}^{3 \times 3}}$. In the following we omit the index $\mathbb{R}^{3}, \mathbb{R}^{3 \times 3}$. The identity tensor on $\mathbb{R}^{3 \times 3}$ will be denoted by $\mathbb{1}$, so that $\operatorname{tr}(\mathbf{X})=\langle\mathbf{X}, \mathbb{1}\rangle$. We let Sym denote the set of symmetric tensors. We adopt the usual abbreviations of Lie-algebra theory, i.e., $\mathfrak{s o}(3):=\left\{\mathbf{A} \in \mathbb{R}^{3 \times 3} \mid \mathbf{A}^{T}=-\mathbf{A}\right\}$ is the Lie-algebra of skew-symmetric tensors and $\mathfrak{s l}(3):=\left\{\mathbf{X} \in \mathbb{R}^{3 \times 3} \mid \operatorname{tr}(\mathbf{X})=0\right\}$ is the Lie-algebra of traceless tensors. For all $\mathbf{X} \in \mathbb{R}^{3 \times 3}$ we set $\operatorname{sym} \mathbf{X}=\frac{1}{2}\left(\mathbf{X}^{T}+\mathbf{X}\right) \in \operatorname{Sym}$, skew $\mathbf{X}=\frac{1}{2}\left(\mathbf{X}-\mathbf{X}^{T}\right) \in \mathfrak{s o}(3)$ and the deviatoric (trace-free) part $\operatorname{dev} \mathbf{X}=\mathbf{X}-\frac{1}{3} \operatorname{tr}(\mathbf{X}) \in \mathfrak{s l}(3)$ and we have the orthogonal Cartan-decomposition of the Liealgebra $\mathfrak{g l}(3)=\{\mathfrak{s l}(3) \cap \operatorname{Sym}(3)\} \oplus \mathfrak{s o}(3) \oplus \mathbb{R} \cdot \mathbb{1}, \mathbf{X}=\operatorname{dev} \operatorname{sym} \mathbf{X}+$ skew $\mathbf{X}+\frac{1}{3} \operatorname{tr}(\mathbf{X}) \mathbb{1}$. We use the canonical identification of $\mathbb{R}^{3}$ with $\mathfrak{s o}(3)$, and, for

$$
\mathbf{A}=\left(\begin{array}{ccc}
0 & -a_{3} & a_{2} \\
a_{3} & 0 & -a_{1} \\
-a_{2} & a_{1} & 0
\end{array}\right) \in \mathfrak{s o}(3)
$$


we consider the operators axl $: \mathfrak{s o}(3) \rightarrow \mathbb{R}^{3}$ and Anti $: \mathbb{R}^{3} \rightarrow \mathfrak{s o}(3)$ through

$$
\begin{aligned}
& \operatorname{axl}(\mathbf{A}):=\left(a_{1}, a_{2}, a_{3}\right)^{T}, \quad \mathbf{A} \cdot v=(\operatorname{axl} \mathbf{A}) \times v, \quad(\operatorname{Anti}(v))_{i j}=-\epsilon_{i j k} v_{k}, \quad \forall v \in \mathbb{R}^{3}, \\
& (\operatorname{axl} \mathbf{A})_{k}=-\frac{1}{2} \epsilon_{i j k} \mathbf{A}_{i j}=\frac{1}{2} \epsilon_{k i j} A_{j i}, \quad A_{i j}=-\epsilon_{i j k}(\operatorname{axl} \mathbf{A})_{k}=: \operatorname{Anti}(\operatorname{axl} \mathbf{A})_{i j},
\end{aligned}
$$

where $\epsilon_{i j k}$ is the totally antisymmetric third order permutation tensor.

For a regular enough function $f\left(t, x_{1}, x_{2}, x_{3}\right), f_{t}$ denotes the derivative with respect to the time $t$, while $\frac{\partial f}{\partial x_{i}}$ denotes the $i$-component of the gradient $\nabla f$. For vector fields $u=\left(u_{1}, u_{2}, u_{3}\right)^{T}$ with $u_{i} \in \mathrm{H}^{1}(\Omega)=$ $\left\{u_{i} \in \mathrm{L}^{2}(\Omega) \mid \nabla u_{i} \in \mathrm{L}^{2}(\Omega)\right\}, i=1,2,3$, we define $\mathrm{D} u:=\left(\nabla u_{1}\left|\nabla u_{2}\right| \nabla u_{3}\right)^{T}$. The corresponding Sobolevspace will be also denoted by $\mathrm{H}^{1}(\Omega)$. In addition, for a tensor field $\mathbf{P}$ with rows in $\mathrm{H}(\operatorname{div} ; \Omega)$, i.e., $\mathbf{P}=$ $\left(\mathbf{P}^{T} . e_{1}\left|\mathbf{P}^{T} . e_{2}\right| \mathbf{P}^{T} e_{3}\right)^{T}$ with $\left(\mathbf{P}^{T} . e_{i}\right)^{T} \in \mathrm{H}(\operatorname{div} ; \Omega):=\left\{v \in \mathrm{L}^{2}(\Omega) \mid \operatorname{div} v \in \mathrm{L}^{2}(\Omega)\right\}, i=1,2,3$, we define $\operatorname{Div} \mathbf{P}:=\left(\operatorname{div}\left(\mathbf{P}^{T} . e_{1}\right)^{T}\left|\operatorname{div}\left(\mathbf{P}^{T} \cdot e_{2}\right)^{T}\right| \operatorname{div}\left(\mathbf{P}^{T} e_{3}\right)^{T}\right)^{T}$ while for tensor fields $\mathbf{P}$ with rows in $\mathrm{H}(\operatorname{curl} ; \Omega)$, i.e., $\mathbf{P}=$ $\left(\mathbf{P}^{T} . e_{1}\left|\mathbf{P}^{T} . e_{2}\right| \mathbf{P}^{T} e_{3}\right)^{T}$ with $\left(\mathbf{P}^{T} . e_{i}\right)^{T} \in \mathrm{H}(\operatorname{curl} ; \Omega):=\left\{v \in \mathrm{L}^{2}(\Omega) \mid \operatorname{curl} v \in \mathrm{L}^{2}(\Omega)\right\}, i=1,2,3$, we define $\operatorname{Curl} \mathbf{P}:=\left(\operatorname{curl}\left(\mathbf{P}^{T} \cdot e_{1}\right)^{T}\left|\operatorname{curl}\left(\mathbf{P}^{T} \cdot e_{2}\right)^{T}\right| \operatorname{curl}\left(\mathbf{P}^{T} e_{3}\right)^{T}\right)^{T}$.

\subsection{Cosserat theory of isotropic elastic solids as particular case of the relaxed micromorphic model}

In this subsection we show that the dynamic Cosserat model for isotropic materials [20, 49] is not only a special case of the most general micromorphic model, but also a special case of the relaxed micromorphic model $[52,44,43,45,51]$. In the micromorphic theory, the micro-distortion tensor $\mathbf{P}=\left(\mathbf{P}_{i j}\right): \Omega \times[0, T] \rightarrow \mathbb{R}^{3 \times 3}$ describes the substructure of the material which can rotate, stretch, shear and shrink, while $u=\left(u_{i}\right): \Omega \times$ $[0, T] \rightarrow \mathbb{R}^{3}$ is the displacement of the macroscopic material points.

In the relaxed micromorphic model, in which the Cosserat modulus $\mu_{\mathrm{c}}>0$ is related to the isotropic Eringen-Claus model for dislocation dynamics [12, 21, 13], the free energy is given by

$$
\begin{gathered}
W_{\text {relax }}=\mu_{\mathrm{e}}\|\operatorname{sym}(\mathrm{D} u-\mathbf{P})\|^{2}+\mu_{\mathrm{c}}\|\operatorname{skew}(\mathrm{D} u-\mathbf{P})\|^{2}+\frac{\lambda_{\mathrm{e}}}{2}[\operatorname{tr}(\mathrm{D} u-\mathbf{P})]^{2}+\mu_{\text {micro }}\|\operatorname{sym} \mathbf{P}\|^{2}+\frac{\lambda_{\text {micro }}}{2}[\operatorname{tr}(\mathbf{P})]^{2} \\
+\frac{\mu_{\mathrm{e}} L_{\mathrm{c}}^{2}}{2}\left[a_{1} \| \text { dev sym Curl } \mathbf{P}\left\|^{2}+a_{2}\right\| \text { skew } \operatorname{Curl} \mathbf{P} \|+\frac{a_{3}}{3} \operatorname{tr}(\operatorname{Curl} \mathbf{P})^{2}\right]
\end{gathered}
$$

where $\left(\mu_{\mathrm{e}}, \lambda_{\mathrm{e}}\right),\left(\mu_{\text {micro }}, \lambda_{\text {micro }}\right), \mu_{\mathrm{c}}, L_{\mathrm{c}}$ and $\left(a_{1}, a_{2}, a_{3}\right)$ are the elastic moduli representing the parameters related to the meso-scale, the parameters related to the micro-scale the Cosserat couple modulus, the characteristic length, and the three general isotropic curvature parameters (weights), respectively. Formally, letting $L_{\mathrm{c}} \rightarrow \infty$ means a "zoom" into the micro-structure while $L_{\mathrm{c}} \rightarrow 0$ means considering arbitrary large bodies while retaining the size of the unit-cell or keeping the dimensions of the body fixed while reducing the dimensions of the unit cell to zero, or, in other words, "no special effects of the microstructure taking into account" (classical elasticity).

In the internal energy is positive definite in terms of the independent constitutive variables $\mathrm{D} u-\mathbf{P}, \operatorname{sym} \mathbf{P}$, Curl $\mathbf{P}$ if and only if

$$
\begin{array}{rlrl}
\mu_{\mathrm{e}} & >0, & \kappa_{\mathrm{e}}:=\frac{2 \mu_{\mathrm{e}}+3 \lambda_{\mathrm{e}}}{3}>0, & \mu_{\mathrm{c}}>0, \\
\mu_{\text {micro }}>0, & \kappa_{\text {micro }}:=\frac{2 \mu_{\text {micro }}+3 \lambda_{\text {micro }}}{3}>0, & \\
a_{1}>0, & a_{2}>0, & a_{3}>0 .
\end{array}
$$

The complete system of linear partial differential equations in terms of the kinematical unknowns $u$ and $P$ is given by

$$
\begin{aligned}
& \rho u_{, t t}=\operatorname{Div}[\underbrace{2 \mu_{\mathrm{e}} \operatorname{sym}(\mathrm{D} u-\mathbf{P})+2 \mu_{\mathrm{c}} \operatorname{skew}(\mathrm{D} u-\mathbf{P})+\lambda_{\mathrm{e}} \operatorname{tr}(\mathrm{D} u-\mathbf{P}) \cdot \mathbb{1}}_{\text {the non-symmetric force-stress tensor }}]+f, \\
& \rho \eta \tau_{\mathrm{c}}^{2} P_{, t t}=-\mu_{\mathrm{e}} L_{\mathrm{c}}^{2} \operatorname{Curl}[\underbrace{a_{1} \operatorname{dev} \operatorname{sym} \operatorname{Curl} \mathbf{P}+a_{2} \operatorname{skew} \operatorname{Curl} \mathbf{P}+\frac{a_{3}}{3} \operatorname{tr}(\operatorname{Curl} \mathbf{P}) \cdot \mathbb{1}}_{\text {the second-order moment stress tensor }}] \\
& +2 \mu_{\mathrm{e}} \operatorname{sym}(\mathrm{D} u-\mathbf{P})+\lambda_{\mathrm{e}} \operatorname{tr}(\mathrm{D} u-\mathbf{P}) \cdot \mathbb{1}-2 \mu_{\text {micro }} \operatorname{sym} \mathbf{P}-\lambda_{\text {micro }} \operatorname{tr}(\mathbf{P}) \cdot \mathbb{1}+\mathbf{M} \quad \text { in } \quad \Omega \times[0, T],
\end{aligned}
$$


where $f: \Omega \times[0, T] \rightarrow \mathbb{R}^{3}$ describes the external body force, $\mathbf{M}: \Omega \times[0, T] \rightarrow \mathbb{R}^{3 \times 3}$ describes the external body moment, $\rho$ is the mass density and $\eta \tau_{\mathrm{c}}^{2}$ is the inertia coefficient, with $\eta>0$ a weight parameter and $\tau_{\mathrm{c}}$ the internal characteristic time [20, page 163].

In the Cosserat theory we assume that the micro-distortion tensor is skew-symmetric, i.e. $\mathbf{P}=\mathbf{A} \in \mathfrak{s o}(3)$. Using the Curl-D axl identities, (see [54], Nye's formula [58])

$$
-\operatorname{Curl} \mathbf{A}=(\mathrm{D} \operatorname{axl} \mathbf{A})^{T}-\operatorname{tr}\left[(\mathrm{D} \operatorname{axl} \mathbf{A})^{T}\right] \cdot \mathbb{1}, \quad \quad \mathrm{D} \operatorname{axl} \mathbf{A}=-(\operatorname{Curl} \mathbf{A})^{T}+\frac{1}{2} \operatorname{tr}\left[(\operatorname{Curl} \mathbf{A})^{T}\right] \cdot \mathbb{1},
$$

for all matrix fields $\mathbf{A} \in \mathfrak{s o}(3)$, it is easy to obtain that the total energies admits the form (identifying $\vartheta=\operatorname{axl} \mathbf{A}$ )

$$
\begin{aligned}
& \mathcal{L}\left(u_{, t},(\operatorname{axl} \mathbf{A})_{, t}, \mathrm{D} u-\mathbf{A}, \operatorname{axl} \mathbf{A}\right) \\
& =\int_{\Omega}\left(\frac{1}{2} \rho\left\|u_{, t}\right\|^{2}+\rho \eta \tau_{\mathrm{c}}^{2}\left\|(\operatorname{axl} \mathbf{A}){ }_{, t}\right\|^{2}+\mu_{\mathrm{e}}\|\operatorname{sym} \mathrm{D} u\|^{2}+\mu_{\mathrm{c}}\|\operatorname{skew}(\mathrm{D} u-\mathbf{A})\|^{2}+\frac{\lambda_{\mathrm{e}}}{2}[\operatorname{tr}(\mathrm{D} u)]^{2}\right. \\
& \left.\quad+\frac{\mu_{\mathrm{e}} L_{\mathrm{c}}^{2}}{2}\left[a_{1}\|\operatorname{dev} \operatorname{sym}(\mathrm{D} \operatorname{axl} \mathbf{A})\|^{2}+a_{2}\|\operatorname{skew}(\mathrm{D} \operatorname{axl} \mathbf{A})\|^{2}+\frac{4 a_{3}}{3}[\operatorname{tr}(\mathrm{D} \operatorname{axl} \mathbf{A})]^{2}\right]\right) d v
\end{aligned}
$$

and lead to Euler-Lagrange equations which are equivalent to those derived from the Curl-formulation.

The power functional is given by

$$
\Pi(t)=\int_{\Omega}\left(\left\langle f, u_{, t}\right\rangle+\left\langle\mathbf{M}, \mathbf{A}_{, t}\right\rangle\right) d v=\int_{\Omega}\left(\left\langle f, u_{, t}\right\rangle+2\left\langle\operatorname{axl} \text { skew } M, \operatorname{axl} \mathbf{A}_{, t}\right\rangle\right) d v .
$$

We introduce the action functional of the considered system to be defined as

$$
\begin{aligned}
\mathcal{A}= & \int_{0}^{T} \int_{\Omega}\left(\frac{1}{2} \rho\left\|u_{, t}\right\|^{2}+\rho \eta \tau_{\mathrm{c}}^{2}\left\|(\operatorname{axl} \mathbf{A})_{, t}\right\|^{2}-\left[\mu_{\mathrm{e}}\|\operatorname{symD} u\|^{2}+\mu_{\mathrm{c}}\|\operatorname{skew}(\mathrm{D} u-\mathbf{A})\|^{2}+\frac{\lambda_{\mathrm{e}}}{2}[\operatorname{tr}(\mathrm{D} u)]^{2}\right]\right. \\
& \left.\quad-\frac{\mu_{\mathrm{e}} L_{\mathrm{c}}^{2}}{2}\left[a_{1}\|\operatorname{dev} \operatorname{sym}(\mathrm{D} \operatorname{axl} \mathbf{A})\|^{2}+a_{2}\|\operatorname{skew}(\mathrm{D} \operatorname{axl} \mathbf{A})\|^{2}+\frac{4 a_{3}}{3}[\operatorname{tr}(\mathrm{D} \operatorname{axl} \mathbf{A})]^{2}\right]\right) d v d t \\
& +\int_{0}^{T} \int_{\Omega}(\langle f, u\rangle+2\langle\operatorname{axl} \text { skew } \mathbf{M}, \operatorname{axl} \mathbf{A}\rangle) d v d t .
\end{aligned}
$$

The condition of vanishing first variation of the action functional can thus be written as

$$
\begin{aligned}
-\int_{0}^{T} \int_{\Omega}(\langle f, \delta u\rangle & +2\langle\operatorname{axl} \operatorname{skew} \mathbf{M}, \operatorname{axl}(\delta \mathbf{A})\rangle) d v d t \\
=\int_{0}^{T} \int_{\Omega} & \left(\rho\left\langle u_{, t},(\delta u)_{, t}\right\rangle+2 \rho \eta \tau_{\mathrm{c}}^{2}\left\langle(\operatorname{axl} \mathbf{A})_{, t},(\operatorname{axl}(\delta \mathbf{A}))_{, t}\right\rangle\right. \\
& -2 \mu_{\mathrm{e}}\langle\operatorname{symD} u, \operatorname{symD} \delta u\rangle-\mu_{\mathrm{c}}\langle\operatorname{skew}(\mathrm{D} u-\mathbf{A}), \operatorname{skew}(\mathrm{D} \delta u-\delta \mathbf{A})\rangle-\lambda_{\mathrm{e}}[\operatorname{tr}(\mathrm{D} u)][\operatorname{tr}(\mathrm{D} \delta u)] \\
& -\frac{\mu_{\mathrm{e}} L_{\mathrm{c}}^{2}}{2} a_{1}\langle\operatorname{dev} \operatorname{sym}(\mathrm{D} \operatorname{axl} \mathbf{A}), \operatorname{dev} \operatorname{sym}(\operatorname{D} \operatorname{axl}(\delta \mathbf{A}))\rangle-\frac{\mu_{\mathrm{e}} L_{\mathrm{c}}^{2}}{2} a_{2}\langle\operatorname{skew}(\mathrm{D} \operatorname{axl} \mathbf{A}), \operatorname{skew}(\mathrm{D} \operatorname{axl}(\delta \mathbf{A}))\rangle \\
& \left.-\frac{\mu_{\mathrm{e}} L_{\mathrm{c}}^{2}}{2} \frac{4 a_{3}}{3}[\operatorname{tr}(\mathrm{D} \operatorname{axl} \mathbf{A})][\operatorname{tr}(\operatorname{D} \operatorname{axl}(\delta \mathbf{A}))]\right) d v d t \\
=\int_{0}^{T} \int_{\Omega} & \left(\rho\left\langle u_{, t},(\delta u)_{, t}\right\rangle+2 \rho \eta \tau_{\mathrm{c}}^{2}\left\langle(\operatorname{axl} \mathbf{A})_{, t},(\operatorname{axl}(\delta \mathbf{A}))_{, t}\right\rangle\right. \\
& -\left\langle 2 \mu_{\mathrm{e}} \operatorname{sym} \mathrm{D} u+\mu_{\mathrm{c}} \operatorname{skew}(\mathrm{D} u-\mathbf{A})+\lambda_{\mathrm{e}}[\operatorname{tr}(\mathrm{D} u)] \cdot \mathbb{1}, \mathrm{D} \delta u\right\rangle-2 \mu_{\mathrm{c}}\langle\operatorname{skew}(\mathrm{D} u-\mathbf{A}), \delta \mathbf{A}\rangle \quad(2.10) \\
& \left.\left.-\frac{\mu_{\mathrm{e}} L_{\mathrm{c}}^{2}}{2}\left\langle a_{1} \operatorname{dev} \operatorname{sym}(\mathrm{D} \operatorname{axl} \mathbf{A})+a_{2} \operatorname{skew}(\mathrm{D} \operatorname{axl} \mathbf{A})+\frac{4 a_{3}}{3}[\operatorname{tr}(\mathrm{D} \operatorname{axl} \mathbf{A})] \cdot \mathbb{1}, \mathrm{D} \operatorname{axl}(\delta \mathbf{A})\right\rangle\right\rangle\right) d v d t \\
=\int_{0}^{T} \int_{\Omega} & \left(-\rho\left\langle u_{, t t}, \delta u\right\rangle-2 \rho \eta \tau_{\mathrm{c}}^{2}\left\langle(\operatorname{axl} \mathbf{A})_{, t t}, \operatorname{axl}(\delta \mathbf{A})\right\rangle\right. \\
& +\left\langle\operatorname{Div}\left[2 \mu_{\mathrm{e}} \operatorname{symD} u+\mu_{\mathrm{c}} \operatorname{skew}(\mathrm{D} u-\mathbf{A})+\lambda_{\mathrm{e}}[\operatorname{tr}(\mathrm{D} u)] \cdot \mathbb{1}\right], \delta u\right\rangle-4 \mu_{\mathrm{c}}\langle\operatorname{axl} \operatorname{skew}(\mathrm{D} u-\mathbf{A}), \operatorname{axl}(\delta \mathbf{A})\rangle
\end{aligned}
$$




$$
\left.\left.+\frac{\mu_{\mathrm{e}} L_{\mathrm{c}}^{2}}{2}\left\langle\operatorname{Div}\left[a_{1} \operatorname{dev} \operatorname{sym}(\operatorname{D} \operatorname{axl} \mathbf{A})+a_{2} \operatorname{skew}(\operatorname{D} \operatorname{axl} \mathbf{A})+\frac{4 a_{3}}{3}[\operatorname{tr}(\operatorname{Daxl} \mathbf{A})] \cdot \mathbb{1}\right], \operatorname{axl}(\delta \mathbf{A})\right\rangle\right\rangle\right) d v d t
$$

for all virtual displacements $\delta u \in C_{0}^{\infty}\left([0, T], C_{0}^{\infty}(\Omega)\right)$ and for all virtual axial vectors axl $(\delta \mathbf{A}) \in C_{0}^{\infty}\left([0, T], C_{0}^{\infty}(\Omega)\right)$. Summarizing, in view of (2.10), the Euler-Lagrange equation gives us the following system of partial differential equations for $u$ and $\mathbf{A}$

$$
\begin{aligned}
\rho u_{, t t}= & \operatorname{Div}\left[2 \mu_{\mathrm{e}} \operatorname{sym} \mathrm{D} u+2 \mu_{\mathrm{c}} \operatorname{skew}(\mathrm{D} u-\mathbf{A})+\lambda_{\mathrm{e}} \operatorname{tr}(\mathrm{D} u) \cdot \mathbb{1}\right]+f, \\
2 \rho \eta \tau_{\mathrm{c}}^{2}(\operatorname{axl} \mathbf{A})_{, t t}= & \frac{\mu_{\mathrm{e}} L_{\mathrm{c}}^{2}}{2}\left[a_{1} \operatorname{dev} \operatorname{sym}(\mathrm{D} \operatorname{axl} \mathbf{A})+a_{2} \operatorname{skew}(\mathrm{D} \operatorname{axl} \mathbf{A})+\frac{4 a_{3}}{3} \operatorname{tr}(\mathrm{D} \operatorname{axl} \mathbf{A}) \cdot \mathbb{1}\right] \\
& -4 \mu_{\mathrm{c}} \operatorname{axl}(\operatorname{skew} \mathrm{D} u-\mathbf{A})+2 \operatorname{axl} \text { skew } \mathbf{M} \quad \text { in } \quad \Omega \times[0, T],
\end{aligned}
$$

which is in complete agreement to the equations proposed in the Cosserat theory [20], but written in indices.

Therefore, in the Cosserat theory of linear elastic materials, two vector fields are used to describe the macroand micro-behaviour of the solid body, i.e., the displacement $u: \Omega \subset \mathbb{R}^{3} \mapsto \mathbb{R}^{3}$ and the microrotation vector field $\vartheta: \Omega \subset \mathbb{R}^{3} \mapsto \mathbb{R}^{3}, \vartheta=\operatorname{axl} \mathbf{A}$, where $\mathbf{A} \in \mathfrak{s o}(3)$ represents the micro-distortion tensor in the Cosserat theory, and within the framework of the linear isotropic hyperelastic theory, the elastic energy density of the Cosserat model read

$$
\begin{aligned}
W(\mathrm{D} u, \operatorname{Anti} \vartheta, \mathrm{D} \vartheta)= & \underbrace{\mu_{\mathrm{e}}\left\|\operatorname{dev}_{3} \operatorname{sym} \mathbf{e}\right\|^{2}+\mu_{\mathrm{c}} \| \text { skew } \mathbf{e} \|^{2}+\frac{2 \mu_{\mathrm{e}}+3 \lambda_{\mathrm{e}}}{6}[\operatorname{tr}(\mathbf{e})]^{2}}_{:=W_{1}(\mathbf{e})} \\
& +\underbrace{\mu_{\mathrm{e}} L_{\mathrm{c}}^{2}\left[\alpha_{1}\left\|\operatorname{dev}_{3} \operatorname{sym} \mathfrak{K}\right\|^{2}+\alpha_{2} \| \text { skew } \mathfrak{K} \|^{2}+\frac{2 \alpha_{1}+3 \alpha_{3}}{6}[\operatorname{tr}(\mathfrak{K})]^{2}\right]}_{:=W_{2}(\mathfrak{K})},
\end{aligned}
$$

where we have used the notations for the weight parameters $\alpha_{1}=\frac{a_{1}}{2}, \alpha_{2}=\frac{a_{2}}{2}$ and $\alpha_{3}=4 \frac{a_{3}}{3}$ and the following definitions for the independent constitutive variables $\mathbf{e}$ and $\mathfrak{K}$ (geometrical equations)

$$
\mathbf{e}:=\mathrm{D} u-\mathbf{A}=\mathrm{D} u-\operatorname{Anti}(\vartheta), \quad \mathfrak{K}:=\mathrm{D} \vartheta .
$$

Hence, the stress-strain relations for the homogeneous isotropic Cosserat elastic solid are

$$
\begin{aligned}
\boldsymbol{\sigma} & :=\frac{\partial W}{\partial \mathbf{e}}=2 \mu_{\mathrm{e}} \operatorname{sym} \mathbf{e}+2 \mu_{\mathrm{c}} \text { skew } \mathbf{e}+\lambda_{\mathrm{e}} \operatorname{tr}(\mathbf{e}) \mathbb{1}, \\
\mathbf{m} & :=\frac{\partial W}{\partial \mathfrak{K}}=\mu_{\mathrm{e}} L_{\mathrm{c}}^{2}\left[2 \alpha_{1} \operatorname{sym} \mathfrak{K}+2 \alpha_{2} \text { skew } \mathfrak{K}+\alpha_{3} \operatorname{tr}(\mathfrak{K}) \mathbb{1}\right],
\end{aligned}
$$

where $\boldsymbol{\sigma}$ is the non-symmetric force stress tensor and $\mathbf{m}$ is the second-order non-symmetric couple stress tensor. In the absence of external body forces and of external body moment, the PDE-system of the model is

$$
\begin{gathered}
\rho \frac{\partial^{2} u_{i}}{\partial t^{2}}=\left(\mu_{\mathrm{e}}+\mu_{\mathrm{c}}\right) \sum_{l=1}^{3} \frac{\partial^{2} u_{i}}{\partial x_{l}^{2}}+\left(\mu_{\mathrm{e}}-\mu_{\mathrm{c}}+\lambda_{\mathrm{e}}\right) \sum_{l=1}^{3} \frac{\partial^{2} u_{l}}{\partial x_{l} \partial x_{i}}+2 \mu_{\mathrm{c}} \sum_{l, s=1}^{3} \varepsilon_{i l s} \frac{\partial \vartheta_{s}}{\partial x_{l}}, \\
\rho j \mu_{\mathrm{e}} \tau_{\mathrm{c}}^{2} \frac{\partial^{2} \vartheta_{i}}{\partial t^{2}}=\mu_{\mathrm{e}} L_{\mathrm{c}}^{2}\left[\left(\alpha_{1}+\alpha_{2}\right) \sum_{l=1}^{3} \frac{\partial^{2} \vartheta_{i}}{\partial x_{l}^{2}}+\left(\alpha_{1}-\alpha_{2}+\alpha_{3}\right) \sum_{l=1}^{3} \frac{\partial^{2} \vartheta_{l}}{\partial x_{l} \partial x_{i}}\right]+2 \mu_{\mathrm{c}} \sum_{l, s=1}^{3} \varepsilon_{i s l} \frac{\partial u_{l}}{\partial x_{s}}-4 \mu_{\mathrm{c}} \vartheta_{i},
\end{gathered}
$$

with $i=1,2,3$, where $j=2 \eta$ is an inertia weight parameter.

Due to the orthogonal Cartan-decomposition of the Lie-algebra $\mathfrak{g l}(3)$, the strict positive definiteness of the potential energy is equivalent to the following simple relations for the introduced parameters

$$
\mu_{\mathrm{e}}>0, \quad \mu_{\mathrm{c}}>0, \quad 2 \mu_{\mathrm{e}}+3 \lambda_{\mathrm{e}}>0, \quad \alpha_{1}>0, \quad \alpha_{2}>0, \quad 2 \alpha_{1}+3 \alpha_{3}>0 .
$$

However, our entire subsequent analysis will be made under weaker conditions on the constitutive parameters.

In the following we assume $\rho>0$ and $j>0$ without mentioning these conditions in the hypothesis of our results. 


\subsection{Real plane waves in isotropic Cosserat elastic solids}

We say that there exists real plane waves in the direction $\xi=\left(\xi_{1}, \xi_{2}, \xi_{3}\right),\|\xi\|^{2}=1$, if for every wave number $k>0$ the system of partial differential equations (2.15) admits a solution in the form:

$$
\begin{aligned}
& u\left(x_{1}, x_{2}, x_{3}, t\right)=\underbrace{\left(\begin{array}{l}
\widehat{u}_{1} \\
\widehat{u}_{2}
\end{array}\right)}_{:=\widehat{u}} e^{\mathrm{i}\left(k\langle\xi, x\rangle_{\mathbb{R}^{3}}-\omega t\right)},
\end{aligned}
$$

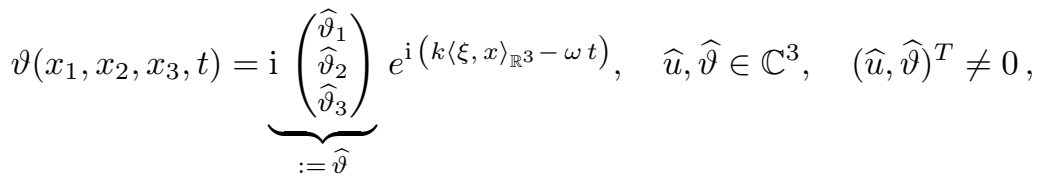

only for real frequencies $\omega \in \mathbb{R}$, where $\mathrm{i}=\sqrt{-1}$ is the complex unit. The plane wave is called "real" since it is defined by real values of $\omega$. Note that we take i $\widehat{\vartheta}$ since this choice will lead us in the end only to real valued matrices. Otherwise, we would have to deal with complex valued matrices in the linear Cosserat theory.

The functions (2.17) are a solution of (2.15) if and only if the following system is satisfied

$$
\begin{aligned}
&-\omega^{2} \rho \widehat{u}_{i}=-k^{2}\left(\mu_{\mathrm{e}}+\mu_{\mathrm{c}}\right) \widehat{u}_{i}-k^{2}\left(\mu_{\mathrm{e}}-\mu_{\mathrm{c}}+\lambda_{\mathrm{e}}\right) \sum_{l=1}^{3} \widehat{u}_{l} \xi_{i} \xi_{l}-2 k \mu_{\mathrm{c}} \sum_{l, s=1}^{3} \varepsilon_{i l s} \widehat{\vartheta}_{s} \xi_{l}, \\
&-\mathrm{i} \omega^{2} \rho j \mu_{\mathrm{e}} \tau_{\mathrm{c}}^{2} \widehat{\vartheta}_{i}=\mu_{\mathrm{e}} L_{\mathrm{c}}^{2}\left[-\mathrm{i} k^{2}\left(\alpha_{1}+\alpha_{2}\right) \widehat{\vartheta}_{i}-\mathrm{i} k^{2}\left(\alpha_{1}-\alpha_{2}+\alpha_{3}\right) \sum_{l=1}^{3} \widehat{\vartheta}_{l} \xi_{l} \xi_{i}\right] \\
&-2 \mathrm{i} k \mu_{\mathrm{c}} \sum_{l, s=1}^{3} \varepsilon_{i l s} \widehat{u}_{s} \xi_{l}-4 \mathrm{i} \mu_{\mathrm{c}} \widehat{\vartheta}_{i}, \quad i=1,2,3 .
\end{aligned}
$$

However, since our formulation is isotropic, by demanding real plane waves in any direction $\xi=\left(\xi_{1}, \xi_{2}, \xi_{3}\right)$, $\|\xi\|=1$, it is equivalent to demand real plane waves in the direction $e_{1}=(1,0,0)$ which means that for all $k>0$ the system

$$
u\left(x_{1}, x_{2}, t\right)=\underbrace{\left(\begin{array}{c}
\widehat{u}_{1} \\
\widehat{u}_{2} \\
\widehat{u}_{3}
\end{array}\right)}_{:=\widehat{u}} e^{\mathrm{i}\left(k x_{1}-\omega t\right)}, \quad \vartheta\left(x_{1}, x_{2}, t\right)=\underbrace{\mathrm{i}\left(\begin{array}{c}
\widehat{\vartheta}_{1} \\
\widehat{\vartheta}_{2} \\
\widehat{\vartheta}_{3}
\end{array}\right)}_{:=\widehat{\vartheta}} e^{\mathrm{i}\left(k x_{1}-\omega t\right)}, \quad \widehat{u}, \widehat{\vartheta} \in \mathbb{C}^{3}, \quad(\widehat{u}, \widehat{\vartheta})^{T} \neq 0
$$

admits non trivial solutions only for real positive values $\omega^{2}$.

Inserting (2.19) into $(2.15)$ we see that $\widehat{u}_{1}, \widehat{u}_{2}$ and $\widehat{\vartheta}_{3}$ have to satisfy the following linear algebraic equations

$$
\begin{aligned}
-\omega^{2} \rho \widehat{u}_{1} & =-k^{2}\left(\mu_{\mathrm{e}}+\mu_{\mathrm{c}}\right) \widehat{u}_{1}-k^{2}\left(\mu_{\mathrm{e}}-\mu_{\mathrm{c}}+\lambda_{\mathrm{e}}\right) \widehat{u}_{1}, \\
-\omega^{2} \rho \widehat{u}_{2} & =-k^{2}\left(\mu_{\mathrm{e}}+\mu_{\mathrm{c}}\right) \widehat{u}_{2}+2 k \mu_{\mathrm{c}} \widehat{\vartheta}_{3}, \\
-\mathrm{i} \omega^{2} \rho j \mu_{\mathrm{e}} \tau_{\mathrm{c}}^{2} \widehat{\vartheta}_{3} & =-\mathrm{i} k^{2} \mu_{\mathrm{e}} L_{\mathrm{c}}^{2}\left(\alpha_{1}+\alpha_{2}\right) \widehat{\vartheta}_{3}-2 \mathrm{i} k \mu_{\mathrm{c}} \widehat{u}_{2}-4 \mathrm{i} \mu_{\mathrm{c}} \widehat{\vartheta}_{3},
\end{aligned}
$$

while $\widehat{u}_{3}, \widehat{\vartheta}_{1}$ and $\widehat{\vartheta}_{2}$ have to satisfy the system of linear equations

$$
\begin{aligned}
-\omega^{2} \rho \widehat{u}_{3} & =-k^{2}\left(\mu_{\mathrm{e}}+\mu_{\mathrm{c}}\right) \widehat{u}_{3}-2 k \mu_{\mathrm{c}} \widehat{\vartheta}_{2}, \\
-\mathrm{i} \omega^{2} \rho j \mu_{\mathrm{e}} \tau_{\mathrm{c}}^{2} \widehat{\vartheta}_{1} & =-\mathrm{i} k^{2} \mu_{\mathrm{e}} L_{\mathrm{c}}^{2}\left(\alpha_{1}+\alpha_{2}\right) \widehat{\vartheta}_{1}-\mathrm{i} k^{2} \mu_{\mathrm{e}} L_{\mathrm{c}}^{2}\left(\alpha_{1}-\alpha_{2}+\alpha_{3}\right) \widehat{\vartheta}_{1}-4 \mathrm{i} \mu_{\mathrm{c}} \widehat{\vartheta}_{1}, \\
-\mathrm{i} \omega^{2} \rho j \mu_{\mathrm{e}} \tau_{\mathrm{c}}^{2} \widehat{\vartheta}_{2} & =-\mathrm{i} k^{2} \mu_{\mathrm{e}} L_{\mathrm{c}}^{2}\left(\alpha_{1}+\alpha_{2}\right) \widehat{\vartheta}_{2}+2 \mathrm{i} k \mu_{\mathrm{c}} \widehat{u}_{3}-4 \mathrm{i} \mu_{\mathrm{c}} \widehat{\vartheta}_{2} .
\end{aligned}
$$

Hence, there exist real plane wave if for every wave number $k>0$ the following systems of equations (2.15) admit non-trivial solutions:

$$
\left[\mathbf{Q}_{1}\left(e_{1}, k\right)-\omega^{2} \widehat{\mathbb{1}}\right] w=0 \quad w=\left(\widehat{u}_{1}, \widehat{u}_{2}, \widehat{\vartheta}_{3}\right)^{T}
$$




$$
\left[\mathbf{Q}_{2}\left(e_{1}, k\right)-\omega^{2} \widetilde{\mathbb{1}}\right] w=0 \quad w=\left(\widehat{u}_{3}, \widehat{\vartheta}_{1}, \widehat{\vartheta}_{2}\right)^{T}
$$

only for real frequencies $\omega \in \mathbb{R}$, where

$$
\begin{aligned}
& \mathbf{Q}_{1}\left(e_{1}, k\right)=\left(\begin{array}{ccc}
k^{2}\left(2 \mu_{\mathrm{e}}+\lambda_{\mathrm{e}}\right) & 0 & 0 \\
0 & k^{2}\left(\mu_{\mathrm{e}}+\mu_{\mathrm{c}}\right) & -2 k \mu_{\mathrm{c}} \\
0 & -2 k \mu_{\mathrm{c}} & k^{2} \mu_{\mathrm{e}} L_{\mathrm{c}}^{2}\left(\alpha_{1}+\alpha_{2}\right)+4 \mu_{\mathrm{c}}
\end{array}\right), \\
& \mathbf{Q}_{2}\left(e_{1}, k\right)=\left(\begin{array}{ccc}
k^{2}\left(\mu_{\mathrm{e}}+\mu_{\mathrm{c}}\right) & 0 & 2 k \mu_{\mathrm{c}} \\
0 & k^{2} \mu_{\mathrm{e}} L_{\mathrm{c}}^{2}\left(2 \alpha_{1}+\alpha_{3}\right)+4 \mu_{\mathrm{c}} & 0 \\
2 k \mu_{\mathrm{c}} & 0 & k^{2} \mu_{\mathrm{e}} L_{\mathrm{c}}^{2}\left(\alpha_{1}+\alpha_{2}\right)+4 \mu_{\mathrm{c}}
\end{array}\right) \text {, } \\
& \widehat{\mathbb{1}}=\left(\begin{array}{ccc}
\rho & 0 & 0 \\
0 & \rho & 0 \\
0 & 0 & \rho j \mu_{\mathrm{e}} \tau_{\mathrm{c}}^{2}
\end{array}\right), \quad \widetilde{\mathbb{1}}=\left(\begin{array}{ccc}
\rho & 0 & 0 \\
0 & \rho j \mu_{\mathrm{e}} \tau_{\mathrm{c}}^{2} & 0 \\
0 & 0 & \rho j \mu_{\mathrm{e}} \tau_{\mathrm{c}}^{2}
\end{array}\right) .
\end{aligned}
$$

In this form, since $\widehat{\mathbb{1}} \neq \mathbb{1}$ and $\widetilde{\mathbb{1}} \neq \mathbb{1}$, these are not eigenvalue problems. However, the system (2.22) is equivalent to

$$
\left[\widehat{\mathbb{1}}^{-1 / 2} \mathbf{Q}_{1}\left(e_{1}, k\right) \widehat{\mathbb{1}}^{-1 / 2}-\omega^{2} \mathbb{1}\right] d=0, \quad d=\widehat{\mathbb{1}}^{1 / 2}\left(\begin{array}{c}
\widehat{u}_{1} \\
\widehat{u}_{2} \\
\widehat{\vartheta}_{3}
\end{array}\right) .
$$

Hence, the system (2.22) is equivalent to the eigenvalue problem

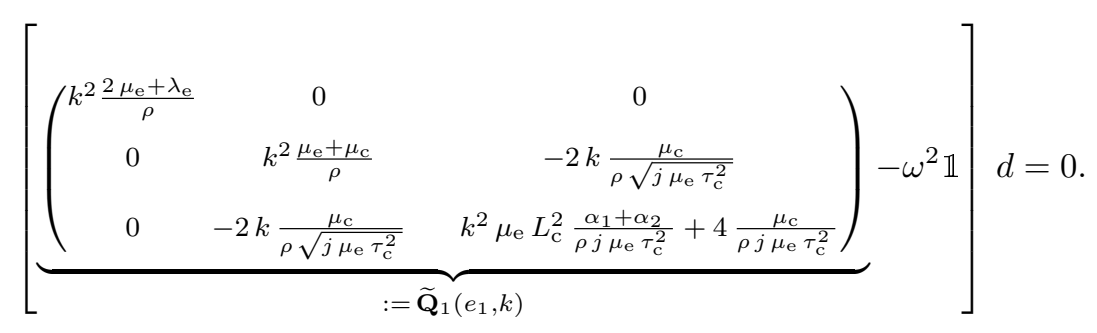

Thus, asking that for all $k>0$ the system $(2.22)_{1}$ admits non trivial solutions $w=\left(\widehat{u}_{1}, \widehat{u}_{2}, \widehat{\vartheta}_{3}\right)^{T} \neq 0$ only for real positive values $\omega^{2}$ is equivalent to the positive definiteness of $\widetilde{\mathbf{Q}}_{1}\left(e_{1}, k\right)$ for all $k>0$. Using the Sylvester criterium this means the following set of conditions

$$
\begin{array}{ll}
k^{2}\left(2 \mu_{\mathrm{e}}+\lambda_{\mathrm{e}}\right)>0, & k^{2}\left(\mu_{\mathrm{e}}+\mu_{\mathrm{c}}\right)>0, \\
\left(\operatorname{det} \widetilde{\mathbf{Q}}_{1}\left(e_{1}, k\right)>0 \quad \Leftrightarrow \quad \mu_{\mathrm{e}} L_{\mathrm{c}}^{2}\left(\alpha_{1}+\alpha_{2}\right) k^{4}\left(\mu_{\mathrm{c}}+\mu_{\mathrm{e}}\right)+k^{2} 4 \mu_{\mathrm{c}} \mu_{\mathrm{e}}>0\right) \quad \forall k>0,
\end{array}
$$

but it also implies that all the diagonal elements are positive, i.e., we also have that

$$
k^{2} \mu_{\mathrm{e}} L_{\mathrm{c}}^{2}\left(\alpha_{1}+\alpha_{2}\right)+4 \mu_{\mathrm{c}}>0 \quad \forall k>0 .
$$

It is now easy to remark that the positive definiteness of $\widetilde{\mathbf{Q}}_{1}\left(e_{1}, k\right)$ for all $k>0$ is equivalent to the non-redundant set of inequalities

$$
2 \mu_{\mathrm{e}}+\lambda_{\mathrm{e}}>0, \quad \mu_{\mathrm{e}}>0, \quad \mu_{\mathrm{c}}>0, \quad \alpha_{1}+\alpha_{2}>0 .
$$

In a similar way, we find that for all $k>0$ the system $(2.22)_{2}$ admits non trivial solutions $w=\left(\widehat{u}_{3}, \widehat{\vartheta}_{1}, \widehat{\vartheta}_{2}\right)^{T} \neq 0$ only for real positive values $\omega^{2}$ if and only if the following eigenvalue problem admits only real solutions

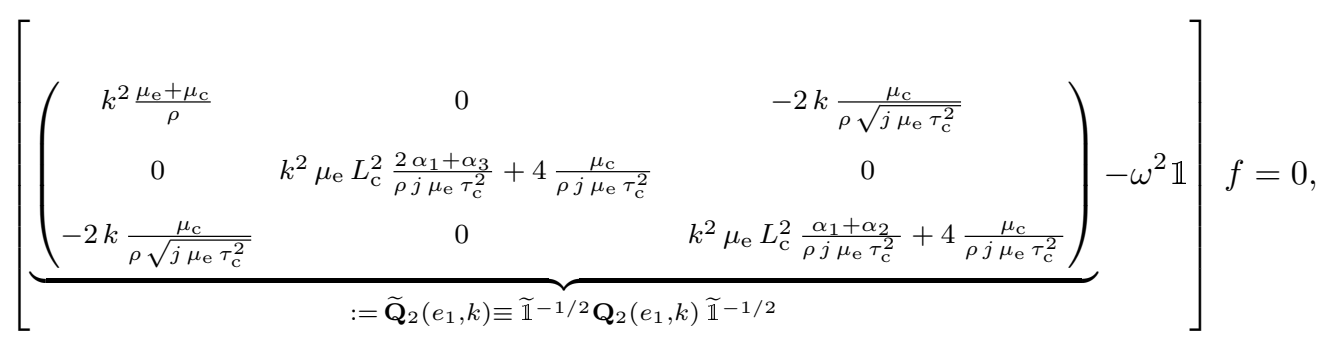


with $f=\widetilde{\mathbb{1}}^{1 / 2}\left(\begin{array}{c}\widehat{u}_{3} \\ \widehat{\vartheta}_{1} \\ \widehat{\vartheta}_{2}\end{array}\right)$, i.e., if and only if for all $k>0$

$$
\begin{array}{ll}
k^{2}\left(\mu_{\mathrm{c}}+\mu_{\mathrm{e}}\right)>0, & 4 \mu_{\mathrm{c}}+\left(2 \alpha_{1}+\alpha_{3}\right) k^{2} L_{\mathrm{c}}^{2}>0, \\
\left(\operatorname{det} \widetilde{\mathbf{Q}}_{2}\left(e_{1}, k\right)>0 \quad \Leftrightarrow \quad\right. & k^{6}\left(\alpha_{1}+\alpha_{2}\right)\left(2 \alpha_{1}+\alpha_{3}\right) L_{\mathrm{c}}^{4} \mu_{\mathrm{e}}\left(\mu_{\mathrm{e}}+\mu_{\mathrm{c}}\right) \\
& \left.+4 k^{4} L_{\mathrm{c}}^{2} \mu_{\mathrm{c}}\left[\left(\alpha_{1}+\alpha_{2}\right) \mu_{\mathrm{c}}\left(\mu_{\mathrm{e}}+\mu_{\mathrm{c}}\right)+\left(2 \alpha_{1}+\alpha_{3}\right) \mu_{\mathrm{e}}\right]+16 k^{2} \mu_{\mathrm{c}}^{2}>0\right),
\end{array}
$$

but it also implies that all the diagonal elements are positive, i.e., we also have that

$$
k^{2} \mu_{\mathrm{e}} L_{\mathrm{c}}^{2}\left(\alpha_{1}+\alpha_{2}\right)+4 \mu_{\mathrm{c}}>0
$$

All together this shows that the positive definiteness of $\widetilde{\mathbf{Q}}_{2}\left(e_{1}, k\right)$ is equivalent to

$$
\mu_{\mathrm{e}}+\mu_{\mathrm{c}}>0, \quad \mu_{\mathrm{c}}>0, \quad 2 \alpha_{1}+\alpha_{3}>0, \quad \mu_{\mathrm{e}}\left(\alpha_{1}+\alpha_{2}\right)>0, \quad \mu_{\mathrm{e}}>0 .
$$

Therefore, the non-redundant inequalities from (2.34) are

$$
\mu_{\mathrm{e}}>0, \quad \mu_{\mathrm{c}}>0, \quad 2 \alpha_{1}+\alpha_{3}>0, \quad\left(\alpha_{1}+\alpha_{2}\right)>0 .
$$

The analysis presented in this subsection is similar to that used in [53]. Then, for $k>0$ and due to the isotropy, extrapolating to all directions of propagation, we have

Proposition 2.1. The necessary and sufficient conditions for existence of real planar waves in any direction $\xi \in \mathbb{R}^{3}, \xi \neq 0$, in the framework of the linear isotropic elastic Cosserat theory are

$$
2 \mu_{\mathrm{e}}+\lambda_{\mathrm{e}}>0, \quad \mu_{\mathrm{e}}>0, \quad \mu_{\mathrm{c}}>0, \quad \alpha_{1}+\alpha_{2}>0, \quad 2 \alpha_{1}+\alpha_{3}>0 .
$$

Proof. The proof is given by the previous calculations.

Remark 2.2. The conditions (2.36) from Proposition 2.1 have also some direct interpretations, i.e.,

i) the first implication of the set of conditions (2.36) means that all these waves (compressional/shearrotational waves, acoustic/optical branch) are real; Once this aspect is clarified, we can treat and interpret further the propagation of plane waves;

ii) under conditions (2.36) all branches of waves are real for the entire range $[0, \infty)$ of the frequency;

iii) we can also see directly from the first condition that the translational compressional wave is real;

iv) the second means that the acoustic branch of shear-rotational wave is real at low frequencies and together with the third means that the acoustic branch of shear-rotational wave is real at high frequencies;

v) the fourth implies that the optical branch of the shear-rotational wave is real at high frequencies;

vi) the third one means that the optical branch of the shear-rotational wave at high frequencies has a larger velocity than the acoustic branch of the same wave at low frequencies (if they both exist, which is the case due to other conditions);

vii) the fifth one expresses directly that the compressional rotational wave at high frequencies is real.

\section{Remark 2.3.}

i) In linear isotropic classical elasticity, the necessary and sufficient conditions for existence of real planar waves in any direction $\xi \in \mathbb{R}^{3}, \xi \neq 0$ are

$$
2 \mu_{\mathrm{e}}+\lambda_{\mathrm{e}}>0, \quad \mu_{\mathrm{e}}>0
$$

and they are equivalent to the strong ellipticity conditions (Legendre-Hadamard ellipticity). 
ii) The necessary and sufficient conditions for existence of a real planar wave are slightly different compared to the strong ellipticity conditions (Legendre-Hadamard ellipticity) (2.36) for the Cosserat (micropolar) model investigated in [2, 19, 61] and which are connected to acceleration waves. In our notation, the strong ellipticity condition for Cosserat media is represented by the inequality [2, 19, 61]

$$
\left.\frac{d}{d \tau} W(\mathbf{e}+\tau \xi \otimes \eta, \mathfrak{K}+\tau \zeta \otimes \eta)\right|_{\tau=0}>0 \quad \forall \eta, \xi, \zeta \in \mathbb{R}^{3}, \quad\|\eta\|=\|\xi\|=\|\zeta\|=1 .
$$

and it is satisfied if and only if

$$
2 \mu_{\mathrm{e}}+\lambda_{\mathrm{e}}>0, \quad \mu_{\mathrm{e}}+\mu_{\mathrm{c}}>0, \quad \alpha_{1}+\alpha_{2}>0, \quad 2 \alpha_{1}+\alpha_{3}>0 .
$$

The explicit calculations in our notations are made in [61]. The absence of a coupling between $\mathbf{e}$ and $\mathfrak{K}$ in the strain energy leads to a simplification of the calculations.

iii) The conditions (2.39) (strong ellipticity conditions, Legendre-Hadamard ellipticity, the positive definiteness of the acoustic tensor) imply the existence of the real translational compressional waves in the entire range of real frequencies, of the real shear rotational waves (both branches) at high frequencies, and of real rotational compressional wave at high frequencies, but at lower frequencies the latter waves may not be real since (2.39) does not guaranty that $\mathfrak{c}_{t}=\frac{\mu_{\mathrm{e}}}{\rho}$ is real. To the contrary, the conditions (2.36) imply that all these branches and types of plane wave are real, i.e., the group/phase velocities are real on the entire range of possible frequencies.

The strong ellipticity conditions (2.39) are weaker than the conditions (2.36) in the sense that they are implied by the necessary and sufficient conditions for existence of a real planar wave (i.e., they imply the strong ellipticity and, therefore, the considered PDEs system is not unstable) but not vice versa. However, the strong ellipticity conditions (2.39) are not sufficient for the application of our solution method, and we believe that they are also not suitable for any approach regarding the propagation of Rayleigh waves in Cosserat solids.

In the end of this section we mention that Eringen [20, pages 149-151] affirmed that one has to impose (in addition) that

$$
\frac{L_{\mathrm{c}}^{2}}{\tau_{\mathrm{c}}^{2}} \frac{\left(\alpha_{1}+\alpha_{2}\right)}{\rho j}>\frac{\mu_{\mathrm{e}}+\mu_{\mathrm{c}}}{\rho},
$$

in order that there exist four real $\omega$ which lead to plane waves solutions (real plane waves). This seems to be only a consequence of the representation formula and the method he used to construct the plane wave solution and seems to be not a necessary condition for real plane waves propagation. Condition (2.40) required by Eringen means that the shearrotational wave at high frequencies is faster for the acoustic branch than for the optical branch. Eringen [20, pages 151] also claimed that this condition is in accordance to the lattice dynamical calculations but no further explanations are given.

Nevertheless, this Eringen-type condition (2.40) seems to have sense when we are going back to the classical linear elasticity model by considering $\mu_{\mathrm{c}} \rightarrow 0$ and large values of $\frac{L_{\mathrm{c}}}{\tau_{\mathrm{c}}}\left(L_{\mathrm{c}} \rightarrow \infty\right.$ or $\left.\tau_{\mathrm{c}} \rightarrow 0\right)$, since for $\xi=e_{1}$ the eigenvalue problems characterising the possible real values of $\omega$ become

$$
\left[\left(\begin{array}{ccc}
k^{2} \frac{2 \mu_{\mathrm{e}}+\lambda_{\mathrm{e}}}{\rho} & 0 & 0 \\
0 & k^{2} \frac{\mu_{\mathrm{e}}}{\rho} & 0 \\
0 & 0 & k^{2} \frac{L_{c}^{2}}{\tau_{c}^{2}} \frac{\alpha_{1}+\alpha_{2}}{\rho j}
\end{array}\right)-\omega^{2} \mathbb{1}\right] d=0, \quad d=\widehat{\mathbb{1}}^{1 / 2}\left(\begin{array}{l}
\widehat{u}_{1} \\
\widehat{u}_{2} \\
\widehat{\vartheta}_{3}
\end{array}\right)
$$

and

$$
\left[\left(\begin{array}{ccc}
k^{2} \frac{\mu_{\mathrm{e}}}{\rho} & 0 & 0 \\
0 & k^{2} \mu_{\mathrm{e}} \frac{2 \alpha_{1}+\alpha_{3}}{\rho j} & 0 \\
0 & 0 & k^{2} \frac{L_{c}^{2}}{\tau_{c}^{2}} \frac{\alpha_{1}+\alpha_{2}}{\rho j}
\end{array}\right)-\omega^{2} \mathbb{1}\right] f=0, \quad f=\widetilde{\mathbb{1}}^{-1 / 2}\left(\begin{array}{c}
\widehat{u}_{3} \\
\widehat{\vartheta}_{1} \\
\widehat{\vartheta}_{2}
\end{array}\right)
$$


In classical linear elasticity, it is natural to impose that plane waves propagate only with speeds $\sqrt{\frac{2 \mu_{\mathrm{e}}+\lambda_{\mathrm{e}}}{\rho}}$ and $\sqrt{\frac{\mu_{\mathrm{e}}}{\rho}}$ and no other "exotic" plane wave arises. Indeed, for $\mu_{\mathrm{c}} \rightarrow 0$ and large values of $\frac{L_{\mathrm{c}}^{2}}{\tau_{\mathrm{c}}^{2}}$, by imposing

$$
\frac{L_{\mathrm{c}}^{2}}{\tau_{\mathrm{c}}^{2}} \min \left\{\frac{2 \alpha_{1}+\alpha_{3}}{\rho j}, \frac{\alpha_{1}+\alpha_{2}}{\rho j}\right\}>\max \left\{\frac{2 \mu_{\mathrm{e}}+\lambda_{\mathrm{e}}}{\rho}, \frac{\mu_{\mathrm{e}}}{\rho}\right\},
$$

the unique propagating speeds which lead to non-vanishing displacements for plane waves are only those from classical elasticity. Condition (2.43) means that both shear-rotational acoustic branch and rotational compressional wave at high frequencies are faster than the shear- rotational acoustic branch at low frequencies and the translational compressional wave. More precisely, under this additional restriction upon the curvature coefficients, in the limit case $\mu_{\mathrm{c}} \rightarrow 0$, we determine the amplitudes

$$
\begin{array}{lrrl}
\text { for } & \omega^{2}=k^{2} \frac{2 \mu_{\mathrm{e}}+\lambda_{\mathrm{e}}}{\rho}: & \widehat{u} \times e_{1}=0, & \widehat{\vartheta}=0, \\
\text { for } & \omega^{2}=k^{2} \frac{\mu_{\mathrm{e}}}{\rho}: & \left\langle\widehat{u}, e_{1}\right\rangle=0, & \widehat{\vartheta}=0, \\
\text { for } & \omega^{2}=k^{2} \frac{L_{\mathrm{c}}^{2}}{\tau_{\mathrm{c}}^{2}} \frac{2 \alpha_{1}+\alpha_{3}}{\rho j}: & \widehat{u}=0, & \widehat{\vartheta} \times e_{1}=0, \\
\text { for } \quad \omega^{2}=k^{2} \frac{L_{\mathrm{c}}^{2}}{\tau_{\mathrm{c}}^{2}} \frac{\alpha_{1}+\alpha_{2}}{\rho j}: & \widehat{u}=0, & \left\langle\widehat{\vartheta}, e_{1}\right\rangle=0 .
\end{array}
$$

This means that real plane waves for which $\widehat{u} \neq 0$ (i.e., only what real plane waves in classical elasticity means) are possible only for $\omega^{2}=k^{2} \sqrt{\frac{2 \mu_{\mathrm{e}}+\lambda_{\mathrm{e}}}{\rho}}$ and $\omega^{2}=k^{2} \sqrt{\frac{\mu_{\mathrm{e}}}{\rho}}$, situation in which the microrotation vector $\widehat{\vartheta}$ vanishes. Moreover, the extremely high values of the frequency which lead to non-vanishing $\widehat{\vartheta}$ are beyond the framework of classical elasticity and belong rather to quantum mechanics or relativistic mechanics.

It is important to note that when studying plane waves, we have to limit the plausible domains of the frequency (i.e., the speed is less than the speed of light) in the framework of classical mechanics, for seismic waves this is already done once the ansatz is chosen, since only subsonic speeds are admissible. We will explain this aspect in more details in the following sections.

\subsection{The setup for the propagation of Rayleigh waves}

In the framework of the Rayleigh wave, we consider the region $\Omega$ to be the half space

$$
\Sigma:=\left\{\left(x_{1}, x_{2}, x_{3}\right) \mid x_{1}, x_{3} \in \mathbb{R}, x_{2} \geq 0\right\} .
$$

The boundary of the homogeneous and isotropic half-space is free of surface traction, i.e.,

$$
\boldsymbol{\sigma} . n=0, \quad \text { m. } n=0 \quad \text { for } \quad x_{2}=0 .
$$

In addition, the solution has to satisfy the following decay condition

$$
\lim _{x_{2} \rightarrow \infty}\left\{u_{1}, u_{2}, \vartheta_{3}, \sigma_{12}, \sigma_{21}, \sigma_{22}, m_{23}\right\}\left(x_{1}, x_{2}, t\right)=0 \quad \forall x_{1} \in \mathbb{R}, \quad \forall t \in[0, \infty) .
$$

In isotropic solids and in the context of Rayleigh wave propagation, the surface particles move in the planes normal to the surface $x_{2}=0$ and parallel to the direction of propagation $e_{1}=(1,0,0)^{T}$, see Figure 1 . Accordingly to these characteristics of the seismic waves, we consider the following plain strain ansatz as a first step in our process of construction of the solution

$$
\begin{aligned}
u\left(x_{1}, x_{2}, t\right)=\left(\begin{array}{c}
u_{1}\left(x_{1}, x_{2}, t\right) \\
u_{2}\left(x_{1}, x_{2}, t\right) \\
0
\end{array}\right), & \mathrm{D} u\left(x_{1}, x_{2}, t\right)=\left(\begin{array}{ccc}
\frac{\partial u_{1}}{\partial x_{1}}\left(x_{1}, x_{2}, t\right) & \frac{\partial u_{2}}{\partial x_{1}}\left(x_{1}, x_{2}, t\right) & 0 \\
\frac{\partial u_{1}}{\partial x_{2}\left(x_{1}, x_{2}, t\right)} & \frac{\partial u_{2}}{\partial x_{2}}\left(x_{1}, x_{2}, t\right) & 0 \\
0 & 0 \\
0 & 0
\end{array}\right) \\
\mathbf{A}\left(x_{1}, x_{2}, t\right)=\left(\begin{array}{ccc}
0 & -\vartheta_{3}\left(x_{1}, x_{2}, t\right) & 0 \\
\vartheta_{3}\left(x_{1}, x_{2}, t\right) & 0 & 0 \\
0 & 0 & 0
\end{array}\right), & \vartheta\left(x_{1}, x_{2}, x_{3}, t\right)=\operatorname{axl} \mathbf{A}=\left(\begin{array}{c}
0 \\
0 \\
\vartheta_{3}\left(x_{1}, x_{2}, t\right)
\end{array}\right) .
\end{aligned}
$$




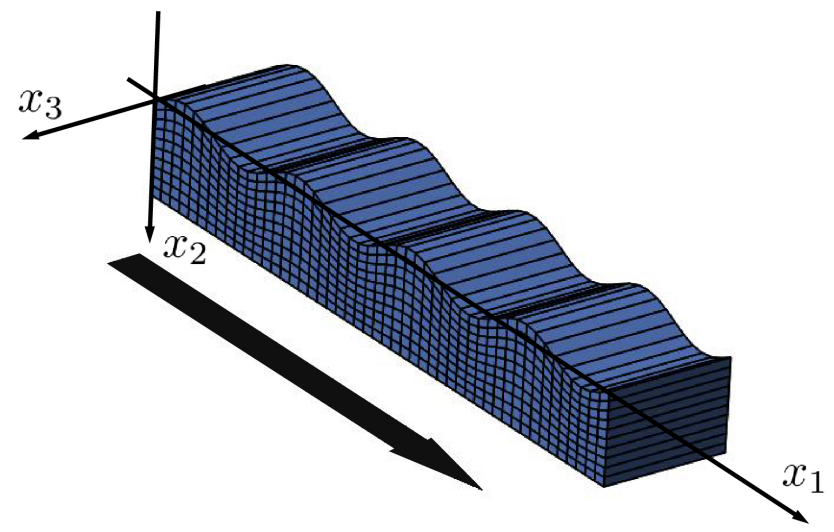

Figure 1: The displacement of a Rayleigh wave, no traction on the upper free surface.

Besides the Rayleigh waves, in the full isotropic Cosserat medium there is also another (transversal, "Love-like") surface wave decribed by $\left(u_{1}, u_{2}, \theta_{3}\right)^{T}=0$ and $\left(u_{3}, \theta_{1}, \theta_{2}\right)^{T} \neq 0$, see [39], while in the reduced Cosserat medium $\left(L_{\mathrm{c}} \rightarrow 0\right)$ there exist non-propagating transversal oscillations of the same kind, see [35]. However, this is not the purpose of the present work and the completed proof of the existence of these "Love-like" surface waves will be considered in the future.

Corresponding to our ansatz, we deduce the following form of the stress tensor

$$
\boldsymbol{\sigma}=\left(\begin{array}{ccc}
\left(2 \mu_{\mathrm{e}}+\lambda_{\mathrm{e}}\right) \frac{\partial u_{1}}{\partial x_{1}}+\lambda_{\mathrm{e}} \frac{\partial u_{2}}{\partial x_{2}} & \left(\mu_{\mathrm{e}}+\mu_{c}\right) \frac{\partial u_{1}}{\partial x_{2}}+2 \mu_{\mathrm{c}} \vartheta_{3}+\left(\mu_{\mathrm{e}}-\mu_{c}\right) \frac{\partial u_{2}}{\partial x_{1}} & 0 \\
\left(\mu_{\mathrm{e}}+\mu_{c}\right) \frac{\partial u_{2}}{\partial x_{1}}-2 \mu_{\mathrm{c}} \vartheta_{3}+\left(\mu_{\mathrm{e}}-\mu_{c}\right) \frac{\partial u_{1}}{\partial x_{2}} & \left(2 \mu_{\mathrm{e}}+\lambda_{\mathrm{e}}\right) u_{2, x_{2}}+\lambda_{\mathrm{e}} \frac{\partial u_{1}}{\partial x_{1}} & 0 \\
0 & 0 & \lambda_{\mathrm{e}}\left(\frac{\partial u_{1}}{\partial x_{1}}+\frac{\partial u_{2}}{\partial x_{2}}\right)
\end{array}\right),
$$

and of the couple stress tensor

$$
\mathbf{m}=\left(\begin{array}{ccc}
0 & 0 & \mu_{\mathrm{e}} L_{\mathrm{c}}^{2}\left(\alpha_{1}-\alpha_{2}\right) \frac{\partial \vartheta_{3}}{\partial x_{1}} \\
0 & 0 & \mu_{\mathrm{e}} L_{\mathrm{c}}^{2}\left(\alpha_{1}-\alpha_{2}\right) \frac{\partial \vartheta_{3}}{\partial x_{2}} \\
\mu_{\mathrm{e}} L_{\mathrm{c}}^{2}\left(\alpha_{1}+\alpha_{2}\right) \frac{\partial \vartheta_{3}}{\partial x_{1}} & \mu_{\mathrm{e}} L_{\mathrm{c}}^{2}\left(\alpha_{1}+\alpha_{2}\right) \frac{\partial \vartheta_{3}}{\partial x_{2}} & 0
\end{array}\right)
$$

while the equation of motion are reduced to

$$
\begin{aligned}
\rho \frac{\partial^{2} u_{1}}{\partial t^{2}} & =\frac{\partial \sigma_{11}}{\partial x_{1}}+\frac{\partial \sigma_{12}}{\partial x_{2}}, \\
\rho \frac{\partial^{2} u_{2}}{\partial t^{2}} & =\frac{\partial \sigma_{22}}{\partial x_{1}}+\frac{\partial \sigma_{22}}{\partial x_{2}}, \\
\rho j \mu_{\mathrm{e}} \tau_{\mathrm{c}}^{2} \frac{\partial^{2} \vartheta_{3}}{\partial t^{2}} & =\frac{\partial m_{31}}{\partial x_{1}}+\frac{\partial m_{32}}{\partial x_{2}}+2 \mu_{\mathrm{c}} \frac{\partial u_{2}}{\partial x_{1}}-2 \mu_{\mathrm{c}} \frac{\partial u_{1}}{\partial x_{2}}-4 \mu_{\mathrm{c}} \vartheta_{3},
\end{aligned}
$$

subjected to the aforementioned boundary conditions (2.45), which turn out to be

$$
\sigma_{12}=0, \quad \sigma_{22}=0, \quad m_{32}=0 \quad \text { at } \quad x_{2}=0 .
$$

Therefore, the aim of this paper is to give an explicit solution $(u, \vartheta)$ of the following system

$$
\begin{aligned}
& \rho \frac{\partial^{2} u_{1}}{\partial t^{2}}=\left(2 \mu_{\mathrm{e}}+\lambda_{\mathrm{e}}\right) \frac{\partial^{2} u_{1}}{\partial x_{1}^{2}}+\lambda_{\mathrm{e}} \frac{\partial^{2} u_{2}}{\partial x_{2} \partial x_{1}}+\left(\mu_{\mathrm{e}}+\mu_{c}\right) \frac{\partial^{2} u_{1}}{\partial x_{2}^{2}}+2 \mu_{\mathrm{c}} \frac{\partial \vartheta_{3}}{\partial x_{2}}+\left(\mu_{\mathrm{e}}-\mu_{c}\right) \frac{\partial^{2} u_{2}}{\partial x_{1} \partial x_{2}}, \\
& \rho \frac{\partial^{2} u_{2}}{\partial t^{2}}=\left(\mu_{\mathrm{e}}+\mu_{c}\right) \frac{\partial^{2} u_{2}}{\partial x_{1}^{2}}-2 \mu_{\mathrm{c}} \frac{\partial \vartheta_{3}}{\partial x_{1}}+\left(\mu_{\mathrm{e}}-\mu_{c}\right) \frac{\partial^{2} u_{1}}{\partial x_{2} \partial x_{1}}+\left(2 \mu_{\mathrm{e}}+\lambda_{\mathrm{e}}\right) \frac{\partial^{2} u_{2}}{\partial x_{2}^{2}}+\lambda_{\mathrm{e}} \frac{\partial^{2} u_{1}}{\partial x_{1} \partial x_{2}},
\end{aligned}
$$




$$
\rho j \mu_{\mathrm{e}} \tau_{\mathrm{c}}^{2} \frac{\partial^{2} \vartheta_{3}}{\partial t^{2}}=\mu_{\mathrm{e}} L_{\mathrm{c}}^{2} \gamma \frac{\partial^{2} \vartheta_{3}}{\partial x_{1}^{2}}+\mu_{\mathrm{e}} L_{\mathrm{c}}^{2} \gamma \frac{\partial^{2} \vartheta_{3}}{\partial x_{2}^{2}}+2 \mu_{\mathrm{c}} \frac{\partial u_{2}}{\partial x_{1}}-2 \mu_{\mathrm{c}} \frac{\partial u_{1}}{\partial x_{2}}-4 \mu_{\mathrm{c}} \vartheta_{3},
$$

which satisfies the boundary conditions at $x_{2}=0$

$$
\begin{aligned}
\left(\mu_{\mathrm{e}}+\mu_{c}\right) \frac{\partial u_{1}}{\partial x_{2}}+2 \mu_{\mathrm{c}} \vartheta_{3}+\left(\mu_{\mathrm{e}}-\mu_{c}\right) \frac{\partial u_{2}}{\partial x_{1}} & =0, \\
\left(2 \mu_{\mathrm{e}}+\lambda_{\mathrm{e}}\right) \frac{\partial u_{2}}{\partial x_{2}}+\lambda_{\mathrm{e}} \frac{\partial u_{1}}{\partial x_{1}} & =0 \\
\mu_{\mathrm{e}} L_{\mathrm{c}}^{2} \gamma \frac{\partial \vartheta_{3}}{\partial x_{2}} & =0
\end{aligned}
$$

where $\gamma=\alpha_{1}+\alpha_{2}$, and which has the asymptotic behaviour (2.46).

Even if until now we have considered the propagation of surface waves with the direction $e_{1}=(1,0,0)^{T}$, i.e. some horizontal direction, $x_{2}$ being the vertical direction orthogonal to the surface along which the wave decays, in one point of our method (see Proposition 3.3 and its implications in Subsection 5) we need to consider a general direction of wave propagation $\xi=\left(\xi_{1}, \xi_{2}, 0\right)^{T}$ and to characterize where the wave is a "real" bulk wave. Therefore, it is useful to know for which conditions on the constitutive parameters (for every wave number $k>0$ and in the direction $\xi=\left(\xi_{1}, \xi_{2}, 0\right)^{T}$ with $\left.\|\xi\|^{2}=1\right)$ the system of partial differential equations (2.15) admits a non trivial solution in the form

$$
\begin{aligned}
& u\left(x_{1}, x_{2}, t\right)=\left(\begin{array}{c}
\widehat{u}_{1} \\
\widehat{u}_{2} \\
0
\end{array}\right) e^{\mathrm{i}\left(k\langle\xi, x\rangle_{\mathbb{R}^{3}}-\omega t\right),} \quad \vartheta\left(x_{1}, x_{2}, t\right)=\mathrm{i}\left(\begin{array}{c}
0 \\
0 \\
\widehat{\vartheta}_{3}
\end{array}\right) e^{\mathrm{i}\left(k\langle\xi, x\rangle_{\mathbb{R}^{3}}-\omega t\right)}, \\
& \left(\widehat{u}_{1}, \widehat{u}_{2}, \widehat{\vartheta_{3}}\right)^{T} \in \mathbb{C}^{3}, \quad\left(\widehat{u}_{1}, \widehat{u}_{2}, \widehat{\vartheta_{3}}\right)^{T} \neq 0
\end{aligned}
$$

only for real positive values $\omega^{2}$. According to the results given in Subsection 2.3, see (2.20), (2.27) and (2.30), we have the following result

Proposition 2.4. Let $\xi \in \mathbb{R}^{3}, \xi \neq 0$ be any direction of the form $\xi=\left(\xi_{1}, \xi_{2}, 0\right)^{T}$. The necessary and sufficient conditions for existence of a non trivial solution of the system of partial differential equations (2.15) of the form given by (2.54) are

$$
2 \mu_{\mathrm{e}}+\lambda_{\mathrm{e}}>0, \quad \mu_{\mathrm{e}}>0, \quad \mu_{\mathrm{c}}>0, \quad \alpha_{1}+\alpha_{2}>0
$$

These restriction (2.55) are the restrictions on the constitutive parameters that we will impose for the rest of the paper. For an interpretation of the conditions (2.55), see Remark 2.2.. These conditions do not involve the constitutive parameter $\alpha_{3}$ and do not imply the existence of real waves in any directions. However, for a given direction $\xi \in \mathbb{R}^{3}, \xi \neq 0$ of the form $\xi=\left(\xi_{1}, \xi_{2}, 0\right)^{T}$, the restrictions (2.55) imply the existence of only real waves defined by expressions of the form (2.54).

\section{The ansatz for the solution and the limiting speed}

We look for a solution of (2.52) and (2.53) having the form ${ }^{3}$

$$
\mathcal{U}\left(x_{1}, x_{2}, t\right)=\left(\begin{array}{l}
u_{1}\left(x_{1}, x_{2}, t\right) \\
u_{2}\left(x_{1}, x_{2}, t\right) \\
\vartheta_{3}\left(x_{1}, x_{2}, t\right)
\end{array}\right)=\operatorname{Re}\left[\left(\begin{array}{c}
z_{1}\left(x_{2}\right) \\
z_{2}\left(x_{2}\right) \\
\mathrm{i} z_{3}\left(x_{2}\right)
\end{array}\right) e^{\mathrm{i} k\left(x_{1}-v t\right)}\right],
$$

where $v$ is the propagation speed (the phase velocity). If $z_{i}, i=1,2,3$, are solutions of the following systems

$$
\begin{gathered}
\left(\begin{array}{ccc}
\mu_{\mathrm{e}}+\mu_{\mathrm{c}} & 0 & 0 \\
0 & 2 \mu_{\mathrm{e}}+\lambda_{\mathrm{e}} & 0 \\
0 & 0 & \mu_{\mathrm{e}} L_{\mathrm{c}}^{2} \gamma
\end{array}\right)\left(\begin{array}{c}
z_{1 \prime \prime}^{\prime \prime}\left(x_{2}\right) \\
z_{2}^{\prime \prime}\left(x_{2}\right) \\
z_{3}^{\prime \prime}\left(x_{2}\right)
\end{array}\right)+\mathrm{i}\left(\begin{array}{ccc}
k\left(\mu_{\mathrm{e}}-\mu_{\mathrm{c}}+\lambda_{\mathrm{e}}\right) & k\left(\mu_{\mathrm{e}}-\mu_{\mathrm{c}}+\lambda_{\mathrm{e}}\right) & 2 \mu_{\mathrm{c}} \\
2 \mu_{\mathrm{c}} & 0 & 0 \\
0 & 0 & 0
\end{array}\right)\left(\begin{array}{c}
z_{1}^{\prime}\left(x_{2}\right) \\
z_{2}^{\prime}\left(x_{2}\right) \\
z_{3}^{\prime}\left(x_{2}\right)
\end{array}\right) \\
\quad-\left(\begin{array}{ccc}
k^{2}\left(2 \mu_{\mathrm{e}}+\lambda_{\mathrm{e}}\right)-\rho k^{2} v^{2} \\
0 & k^{2}\left(\mu_{\mathrm{e}}+\mu_{\mathrm{c}}\right)-\rho k^{2} v^{2} & 0 \\
0 & -2 \mu_{\mathrm{c}} k & k^{2} \mu_{\mathrm{e}} L_{\mathrm{c}}^{2} \gamma+4 \mu_{\mathrm{c}}-j \mu_{\mathrm{e}} \tau_{\mathrm{c}}^{2} k^{2} v^{2}
\end{array}\right)\left(\begin{array}{l}
z_{1}\left(x_{2}\right) \\
z_{2}\left(x_{2}\right) \\
z_{3}\left(x_{2}\right)
\end{array}\right)=0,
\end{gathered}
$$

\footnotetext{
${ }^{3}$ We take $\mathrm{i} z_{3}$ since this choice leads us, in the end, only to real matrices.
} 
and (from the boundary conditions)

$$
\left(\begin{array}{ccc}
\mu_{\mathrm{e}}+\mu_{\mathrm{c}} & 0 & 0 \\
0 & 2 \mu_{\mathrm{e}}+\lambda_{\mathrm{e}} & 0 \\
0 & 0 & \mu_{\mathrm{e}} L_{\mathrm{c}}^{2} \gamma
\end{array}\right)\left(\begin{array}{c}
z_{1}^{\prime}(0) \\
z_{2}^{\prime}(0) \\
z_{3}^{\prime}(0)
\end{array}\right)+\mathrm{i}\left(\begin{array}{ccc}
0 & k\left(\mu_{\mathrm{e}}-\mu_{\mathrm{c}}\right) & 2 \mu_{\mathrm{c}} \\
k \lambda_{\mathrm{e}} & 0 & 0 \\
0 & 0 & 0
\end{array}\right)\left(\begin{array}{c}
z_{1}(0) \\
z_{2}(0) \\
z_{3}(0)
\end{array}\right)=0
$$

where ${ }^{\prime}$ denotes the derivative with respect to $x_{2}$, then $\mathcal{U}$ given by the ansatz (3.1) satisfies (2.52) and (2.53). In a more compact notation, the above equations admit the following equivalent form

$$
\begin{aligned}
\frac{1}{k^{2}} \mathbf{T} z^{\prime \prime}\left(x_{2}\right)+\mathrm{i} \frac{1}{k}\left(\mathbf{R}+\mathbf{R}^{T}\right) z^{\prime}\left(x_{2}\right)-\mathbf{Q} z\left(x_{2}\right)+k^{2} v^{2} \hat{\mathbb{1}} z\left(x_{2}\right) & =0, \\
\frac{1}{k^{2}} \mathbf{T} z^{\prime}(0)+\mathrm{i} \frac{1}{k} \mathbf{R}^{T} z(0) & =0,
\end{aligned}
$$

where the matrices $\mathbf{T}, \mathbf{R}$ and $\mathbf{Q}$ are defined by

$$
\begin{aligned}
\mathbf{T} & =k^{2}\left(\begin{array}{ccc}
\mu_{\mathrm{e}}+\mu_{\mathrm{c}} & 0 & 0 \\
0 & 2 \mu_{\mathrm{e}}+\lambda_{\mathrm{e}} & 0 \\
0 & 0 & \mu_{\mathrm{e}} L_{\mathrm{c}}^{2} \gamma
\end{array}\right), \quad \mathbf{R}=k\left(\begin{array}{ccc}
0 & k \lambda_{\mathrm{e}} & 0 \\
k\left(\mu_{\mathrm{e}}-\mu_{\mathrm{c}}\right) & 0 & 0 \\
2 \mu_{\mathrm{c}} & 0 & 0
\end{array}\right), \\
\mathbf{Q} & =\left(\begin{array}{ccc}
k^{2}\left(2 \mu_{\mathrm{e}}+\lambda_{\mathrm{e}}\right) & 0 & 0 \\
0 & k^{2}\left(\mu_{\mathrm{e}}+\mu_{\mathrm{c}}\right) & -2 \mu_{\mathrm{c}} k \\
0 & -2 \mu_{\mathrm{c}} k & k^{2} \mu_{\mathrm{e}} L_{\mathrm{c}}^{2} \gamma+4 \mu_{\mathrm{c}}
\end{array}\right) .
\end{aligned}
$$

The system (3.3) has a similar structure to that from classical linear elasticity [24] but we still have to rewrite it in order to make it manageable for our analysis. In this respect, the solution $z$ of (3.3) is equivalent to find a solution $y$ of

$$
\begin{array}{r}
\frac{1}{k^{2}} \widehat{\mathbb{1}}^{-1 / 2} \mathbf{T} \widehat{\mathbb{1}}^{-1 / 2} y^{\prime \prime}\left(x_{2}\right)+\mathrm{i} \frac{1}{k} \widehat{\mathbb{1}}^{-1 / 2}\left(\mathbf{R}+\mathbf{R}^{T}\right) \widehat{\mathbb{1}}^{-1 / 2} y^{\prime}\left(x_{2}\right)-\widehat{\mathbb{1}}^{-1 / 2} \mathbf{Q} \widehat{\mathbb{1}}^{-1 / 2} y\left(x_{2}\right)+k^{2} v^{2} \mathbb{1} y\left(x_{2}\right)=0, \\
\frac{1}{k^{2}} \widehat{\mathbb{1}}^{-1 / 2} \mathbf{T} \widehat{\mathbb{1}}^{-1 / 2} y^{\prime}(0)+\mathrm{i} \frac{1}{k} \widehat{\mathbb{1}}^{-1 / 2} \mathbf{R}^{T} \widehat{\mathbb{1}}^{-1 / 2} y(0)=0,
\end{array}
$$

where $y\left(x_{2}\right):=\widehat{\mathbb{1}}^{1 / 2} z\left(x_{2}\right)$. Therefore we use the modified matrices

$$
\begin{aligned}
& \mathcal{T}:=k^{2}\left(\begin{array}{ccc}
\frac{\mu_{\mathrm{e}}+\mu_{\mathrm{c}}}{\rho} & 0 & 0 \\
0 & \frac{2 \mu_{\mathrm{e}}+\lambda_{\mathrm{e}}}{\rho} & 0 \\
0 & 0 & \frac{\mu_{\mathrm{e}} L_{\mathrm{c}}^{2} \gamma}{\rho j \mu_{\mathrm{e}} \tau_{\mathrm{c}}^{2}}
\end{array}\right), \quad \mathcal{R}:=k\left(\begin{array}{ccc}
0 & k \frac{\lambda_{\mathrm{e}}}{\rho} & 0 \\
k \frac{\mu_{\mathrm{e}}-\mu_{\mathrm{c}}}{\rho} & 0 & 0 \\
2 \frac{\mu_{\mathrm{c}}}{\rho \sqrt{j} \mu_{\mathrm{e}} \tau_{\mathrm{c}}^{2}} & 0 & 0
\end{array}\right), \\
& \mathcal{Q}:=\left(\begin{array}{ccc}
k^{2} \frac{2 \mu_{\mathrm{e}}+\lambda_{\mathrm{e}}}{\rho}-k^{2} v^{2} & 0 & 0 \\
0 & k^{2} \frac{\mu_{\mathrm{e}}+\mu_{\mathrm{c}}}{\rho}-k^{2} v^{2} & -2 k \frac{\mu_{\mathrm{c}}}{\rho \sqrt{j \mu_{\mathrm{e}} \tau_{\mathrm{c}}^{2}}} \\
0 & -2 k \frac{\mu_{\mathrm{c}}}{\rho \sqrt{j \mu_{\mathrm{e}} \tau_{\mathrm{c}}^{2}}} & k^{2} \frac{\mu_{\mathrm{e}} L_{\mathrm{c}}^{2} \gamma}{\rho j \mu_{\mathrm{e}} \tau_{\mathrm{c}}^{2}}+4 \frac{\mu_{\mathrm{c}}}{\rho j \mu_{\mathrm{e}} \tau_{\mathrm{c}}^{2}}-k^{2} v^{2}
\end{array}\right),
\end{aligned}
$$

and the following equivalent form of the system (3.3)

$$
\begin{aligned}
\frac{1}{k^{2}} \mathcal{T} y^{\prime \prime}\left(x_{2}\right)+\mathrm{i} \frac{1}{k}\left(\mathcal{R}+\mathcal{R}^{T}\right) y^{\prime}\left(x_{2}\right)-\mathcal{Q} y\left(x_{2}\right)+k^{2} v^{2} \mathbb{1} y\left(x_{2}\right) & =0, \\
\frac{1}{k^{2}} \mathcal{T} y^{\prime}(0)+\mathrm{i} \frac{1}{k} \mathcal{R}^{T} y(0) & =0 .
\end{aligned}
$$

Lemma 3.1. If the constitutive coefficients satisfy the conditions (2.55), then the matrices $\mathcal{Q}$ and $\mathcal{T}$ are symmetric and positive definite.

Proof. Symmetry is clear. It is easy to see that

$$
\begin{aligned}
\mathbf{T}_{11} & =k^{2}\left(\mu_{\mathrm{e}}+\mu_{\mathrm{c}}\right), \quad \mathbf{T}_{11} \mathbf{T}_{22}-\mathbf{T}_{12} \mathbf{T}_{21}=k^{4}\left(2 \mu_{\mathrm{e}}+\lambda_{\mathrm{e}}\right)\left(\mu_{\mathrm{e}}+\mu_{\mathrm{c}}\right), \\
\operatorname{det} \mathbf{T} & =k^{6}\left(2 \mu_{\mathrm{e}}+\lambda_{\mathrm{e}}\right)\left(\mu_{\mathrm{e}}+\mu_{\mathrm{c}}\right) \mu_{\mathrm{e}} L_{\mathrm{c}}^{2} \gamma .
\end{aligned}
$$

Therefore, our constitutive hypothesis imply that $\mathbf{T}$ is positive-definite. In addition, $\mathbf{Q}$ is positive-definite if and only if the principal minors are positive, namely

$$
\mathbf{Q}_{11}=k^{2}\left(2 \mu_{\mathrm{e}}+\lambda_{\mathrm{e}}\right), \quad \mathbf{Q}_{11} \mathbf{Q}_{22}-\mathbf{Q}_{12} \mathbf{Q}_{21}=k^{4}\left(2 \mu_{\mathrm{e}}+\lambda_{\mathrm{e}}\right)\left(\mu_{\mathrm{e}}+\mu_{\mathrm{c}}\right),
$$




$$
\operatorname{det}(\mathbf{Q})=k^{4}\left(2 \mu_{\mathrm{e}}+\lambda_{\mathrm{e}}\right)\left[\left(\mu_{\mathrm{e}}+\mu_{\mathrm{c}}\right) k^{2} \mu_{\mathrm{e}} L_{\mathrm{c}}^{2} \gamma+4 \mu_{\mathrm{e}} \mu_{\mathrm{c}}\right]
$$

i.e. under the hypothesis of the lemma. Since $\mathbf{T}$ and $\mathbf{Q}$ are positive definite, so there are $\mathcal{T}$ and $\mathcal{Q}$ defined by (3.7), and the proof is complete.

We now seek a solution $y$ of the differential system (3.8) in the form

$$
y\left(x_{2}\right)=\left(\begin{array}{l}
d_{1} \\
d_{2} \\
d_{3}
\end{array}\right) e^{\mathrm{i} r k x_{2}}, \quad \operatorname{Im} r>0,
$$

where $r \in \mathbb{C}$ is a complex parameter, $d=\left(d_{1}, d_{2}, d_{3}\right)^{T} \in \mathbb{C}^{3}, a \neq 0$ is the amplitude and $\operatorname{Im} r$ is the coefficient of the imaginary part of $r$. The condition $\operatorname{Im} r>0$ ensures the asymptotic decay condition (2.46). Inserting (3.11) in $(3.3)_{1}$ we obtain the systems of algebraic equations

$$
\left[r^{2} \mathcal{T}+r\left(\mathcal{R}+\mathcal{R}^{T}\right)+\mathcal{Q}-k^{2} v^{2} \mathbb{1}\right] d=0, \quad\left[r \mathcal{T}+\mathcal{R}^{T}\right] d=0
$$

The characteristic equation corresponding to the eigenvalue problem $(3.12)_{1}$, i.e., the condition to have a nontrivial solution of $d=\left(d_{1}, d_{2}, d_{3}\right)^{T}$, is

$$
\operatorname{det}\left[r^{2} \mathcal{T}+r\left(\mathcal{R}+\mathcal{R}^{T}\right)+\mathcal{Q}-k^{2} v^{2} \mathbb{1}\right]=0
$$

which gives six roots of the eigenvalue $r$. The associated eigenvectors $a$ can be determined for the corresponding eigenvalues.

Definition 3.2. By the limiting speed we understand a speed $\widehat{v}>0$, such that for all wave speeds satisfying $0 \leq v<\widehat{v}$ (subsonic speeds) the roots of the characteristic equation (3.13) are not real ${ }^{4}$ and vice versa, i.e., if the roots of the characteristic equation (3.13) are not real then they correspond to wave speeds $v$ satisfying $0 \leq v<\widehat{v}$.

Proposition 3.3. If the constitutive coefficients satisfy the conditions (2.55), then there exists a limiting speed $\widehat{v}>0$. Furthermore, if one root $r_{v}$ of the characteristic equation (3.13) is real then it corresponds to a speed $v \geq \widehat{v}$ (non-admissible).

Proof. Assume that there exists a real $r_{v}$ as solution of the characteristic equation (3.13), then $\exists \theta \in\left(-\frac{\pi}{2}, \frac{\pi}{2}\right)$ such that $r_{v}=\tan \theta$. Therefore, corresponding to (3.11), $\mathcal{U}$ given by (3.1) and defined by $r_{v}$ turns into

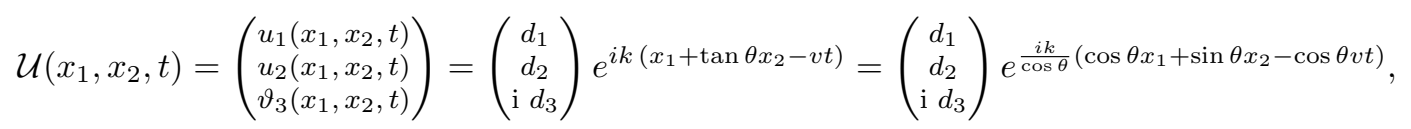

which means that $\mathcal{U}\left(x_{1}, x_{2}, t\right)$ is a non-trivial plane body wave solution with wave number $\frac{k}{\cos \theta}$, the speed $\widetilde{v}_{\theta}=v \cos \theta$ and propagation in the direction $n_{\theta}$ where $n_{\theta}=(\cos \theta, \sin \theta, 0)$. A direct substitution of (3.14) into (2.18) implies the existence of a non-trivial solution $\left(d_{1}, d_{2}, d_{3}\right) \neq 0$ of the algebraic system

$$
\begin{array}{r}
k^{2} \sin ^{2} \theta\left(\mu_{\mathrm{e}}+\mu_{\mathrm{c}}\right) d_{1}+k \sin \theta \cos \theta\left[k \lambda_{\mathrm{e}}+k\left(\mu_{\mathrm{e}}-\mu_{\mathrm{c}}\right)\right] d_{2}+2 \mu_{\mathrm{c}} \sin \theta \cos \theta d_{3}+\cos ^{2} \theta\left[k^{2}\left(2 \mu_{\mathrm{e}}+\lambda_{\mathrm{e}}\right)-k^{2} \rho v^{2}\right] d_{1}=0, \\
k^{2} \sin ^{2} \theta\left(2 \mu_{\mathrm{e}}+\lambda_{\mathrm{e}}\right) d_{2}+k \sin \theta \cos \theta\left[k \lambda_{\mathrm{e}}+k\left(\mu_{\mathrm{e}}-\mu_{\mathrm{c}}\right)\right] d_{1}-2 \mu_{\mathrm{c}} k \cos ^{2} \theta d_{3}+\cos ^{2} \theta\left[k^{2}\left(\mu_{\mathrm{e}}+\mu_{\mathrm{c}}\right)-k^{2} \rho v^{2}\right] d_{2}=0, \\
k^{2} \sin ^{2} \theta \mu_{\mathrm{e}} L_{\mathrm{c}}^{2} \gamma d_{3}+2 \mu_{\mathrm{c}} k \sin \theta \cos \theta d_{1}-2 \mu_{\mathrm{c}} k \cos ^{2} \theta d_{2}+\cos ^{2} \theta\left[k^{2} \mu_{\mathrm{e}} L_{\mathrm{c}}^{2} \gamma+4 \mu_{\mathrm{c}}-\rho j \mu_{\mathrm{e}} \tau_{\mathrm{c}}^{2} k^{2} v^{2}\right] d_{3}=0,
\end{array}
$$

which in matrix form gives

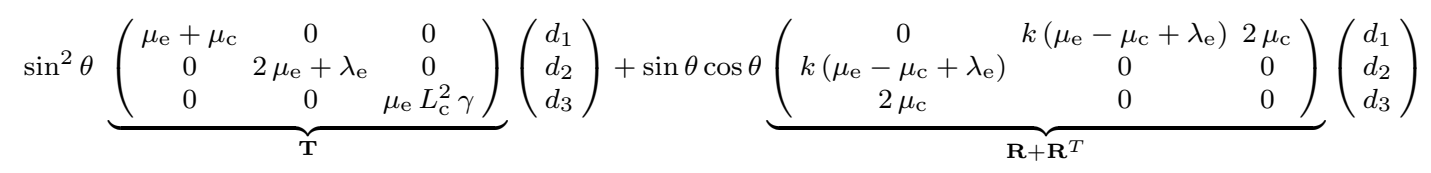

$$
\begin{aligned}
& +\cos ^{2} \theta \underbrace{\left(\begin{array}{ccc}
k^{2}\left(2 \mu_{\mathrm{e}}+\lambda_{\mathrm{e}}\right)-\rho k^{2} v^{2} & 0 \\
0 & k^{2}\left(\mu_{\mathrm{e}}+\mu_{\mathrm{c}}\right)-\rho k^{2} v^{2} & -2 \mu_{\mathrm{c}} k \\
0 & -2 \mu_{\mathrm{c}} k & k^{2} \mu_{\mathrm{e}} L_{\mathrm{c}}^{2} \gamma+4 \mu_{\mathrm{c}}-\rho j \mu_{\mathrm{e}} \tau_{\mathrm{c}}^{2} k^{2} v^{2}
\end{array}\right)}_{\mathbf{Q}-k^{2} v^{2} \widehat{\mathbb{1}}}\left(\begin{array}{l}
d_{1} \\
d_{2} \\
d_{3}
\end{array}\right)=0
\end{aligned}
$$

\footnotetext{
${ }^{4}$ Since the quadratic equation does not have real solutions, there is a complex solution $r$ for which $\operatorname{Im} r>0$, since the complex solutions are pair-conjugated. Therefore, the existence of such a solution $r$, i.e., $\operatorname{Im} r>0$, implies the existence of a wave propagating in the direction $x_{1}$ with the phase velocity $v$ and decaying exponentially in the direction $x_{2}$.
} 
and this is equivalent to

$$
\begin{aligned}
& {\left[k^{2} \sin ^{2} \theta \widehat{\mathbb{1}}^{-1 / 2}\left(\begin{array}{ccc}
\mu_{\mathrm{e}}+\mu_{\mathrm{c}} & 0 & 0 \\
0 & 2 \mu_{\mathrm{e}}+\lambda_{\mathrm{e}} & 0 \\
0 & 0 & \mu_{\mathrm{e}} L_{\mathrm{c}}^{2} \gamma
\end{array}\right) \widehat{\mathbb{1}}^{-1 / 2}+k \sin \theta \cos \theta \widehat{\mathbb{1}}^{-1 / 2}\left(\begin{array}{ccc}
0 & k\left(\mu_{\mathrm{e}}-\mu_{\mathrm{c}}+\lambda_{\mathrm{e}}\right) 2 \mu_{\mathrm{c}} \\
\left.2-\mu_{\mathrm{c}}+\lambda_{\mathrm{e}}\right) & 0 & 0 \\
2 \mu_{\mathrm{c}} & 0 & 0
\end{array}\right) \widehat{\mathbb{1}}^{-1 / 2}\right.} \\
& \left.+\cos ^{2} \theta \widehat{\mathbb{1}}^{-1 / 2}\left(\begin{array}{ccc}
k^{2}\left(2 \mu_{\mathrm{e}}+\lambda_{\mathrm{e}}\right)-\rho k^{2} v^{2} & 0 & 0 \\
0 & k^{2}\left(\mu_{\mathrm{e}}+\mu_{\mathrm{c}}\right)-\rho k^{2} v^{2} & -2 \mu_{\mathrm{c}} k \\
0 & -2 \mu_{\mathrm{c}} k & k^{2} \mu_{\mathrm{e}} L_{\mathrm{c}}^{2} \gamma+4 \mu_{\mathrm{c}}-\rho j \mu_{\mathrm{e}} \tau_{\mathrm{c}}^{2} k^{2} v^{2}
\end{array}\right) \widehat{\mathbb{1}}^{-1 / 2}\right]\left(\begin{array}{l}
f_{1} \\
f_{2} \\
f_{3}
\end{array}\right)=0
\end{aligned}
$$

where $\left(\begin{array}{l}f_{1} \\ f_{2} \\ f_{3}\end{array}\right)=\widehat{\mathbb{1}}^{1 / 2}\left(\begin{array}{l}d_{1} \\ d_{2} \\ d_{3}\end{array}\right) \neq 0$.

Therefore, the assumption from the beginning of the proof implies that there exists a solution $\left(f_{1}, f_{2}, f_{3}\right) \neq 0$ of the algebraic system written in matrix format

$$
\left[\sin ^{2} \theta \boldsymbol{T}+\sin \theta \cos \theta\left(\mathcal{R}+\mathcal{R}^{T}\right)+\cos ^{2} \theta \mathcal{Q}-k^{2} v_{\theta}^{2} \cos ^{2} \theta \mathbb{1}\right]\left(\begin{array}{l}
f_{1} \\
f_{2} \\
f_{3}
\end{array}\right)=0
$$

Let us observe that equation (3.16) is actually the propagation condition for plane waves in isotropic Cosserat materials, in the fixed direction $n_{\theta}=(\cos \theta, \sin \theta, 0)$. Since the constitutive coefficients satisfy the conditions (2.55), according to Proposition 2.4, for the direction $n_{\theta}=(\cos \theta, \sin \theta, 0)$ in particular, the system of partial differential equations (2.15) admits a non trivial solution in the form

$$
\begin{aligned}
& u\left(x_{1}, x_{2}, t\right)=\left(\begin{array}{c}
\widehat{u}_{1} \\
\widehat{u}_{2} \\
0
\end{array}\right) e^{\mathrm{i}\left(k\langle\xi, x\rangle_{\mathbb{R}^{3}}-\omega t\right)}, \quad \vartheta\left(x_{1}, x_{2}, t\right)=\mathrm{i}\left(\begin{array}{c}
0 \\
0 \\
\widehat{\vartheta}_{3}
\end{array}\right) e^{\mathrm{i}\left(k\langle\xi, x\rangle_{\mathbb{R}^{3}}-\omega t\right)}, \\
& \left(\widehat{u}_{1}, \widehat{u}_{2}, \widehat{\vartheta_{3}}\right)^{T} \in \mathbb{C}^{3}, \quad\left(\widehat{u}_{1}, \widehat{u}_{2}, \widehat{\vartheta_{3}}\right)^{T} \neq 0
\end{aligned}
$$

only for real positive values $\omega^{2}$. To each $\theta \in\left(-\frac{\pi}{2}, \frac{\pi}{2}\right)$ we associate these real frequencies $\omega_{\theta}$ satisfying

$$
\operatorname{det}\left\{\sin ^{2} \theta \mathcal{T}+\sin \theta \cos \theta\left(\mathcal{R}+\mathcal{R}^{T}\right)+\cos ^{2} \theta \mathcal{Q}-\omega_{\theta}^{2} \mathbb{1}\right\}=0 .
$$

Then, each $\omega_{\theta}$ defines a $v_{\theta}$ such that $\omega_{\theta}=v_{\theta} \cos \theta$. We define $\widehat{v}$ as the minimum of the values of $v_{\theta} \cos \theta$ for all $\theta \in\left(-\frac{\pi}{2}, \frac{\pi}{2}\right)$. Hence, this means that we find all solutions $v_{\theta}$ of

$$
\operatorname{det}\left\{\sin ^{2} \theta \mathcal{T}+\sin \theta \cos \theta\left(\mathcal{R}+\mathcal{R}^{T}\right)+\cos ^{2} \theta \mathcal{Q}-k^{2} v_{\theta}^{2} \cos ^{2} \theta \mathbb{1}\right\}=0,
$$

and we define $e^{5}$

$$
\widehat{v}=\inf _{\theta \in\left(-\frac{\pi}{2}, \frac{\pi}{2}\right)} v_{\theta} .
$$

In conclusion, if there exists a value of $v$ such that the equation (3.13) admits a real solution $r_{v}$, then $v$ must satisfy $v \geq \widehat{v}$. Thus, if $v$ is such that $0 \leq v<\widehat{v}$, then $r$ can not be real and if $r$ is real, then $v \geq \widehat{v}$.

Proposition 3.4. If the constitutive coefficients satisfy the conditions (2.55), then for all $\theta \in\left(-\frac{\pi}{2}, \frac{\pi}{2}\right)$ and $k>0$, the tensor $\mathcal{Q}_{\theta}:=\sin ^{2} \theta \mathcal{T}+\sin \theta \cos \theta\left(\mathcal{R}+\mathcal{R}^{T}\right)+\cos ^{2} \theta \mathcal{Q}$ is positive definite.

Proof. Since the constitutive coefficients satisfy the conditions (2.55), according to Proposition 2.4, it follows that there exists only real numbers $\omega$ such that the following system ${ }^{6}$ admits non trivial solutions

\footnotetext{
${ }^{5}$ In others words, we can take the solution $v_{b}(\theta)$ of all possible plane body waves propagating in the direction $\widehat{n}$ and we take
}

$$
\widehat{v}=\inf _{\theta \in\left(-\frac{\pi}{2}, \frac{\pi}{2}\right)} \frac{v_{b}(\theta)}{\cos \theta} .
$$

${ }^{6}$ This system follows directly from (2.18). Inserting (2.17) into (2.15) we see that if $\widehat{u}_{1}, \widehat{u}_{2}$ and $\widehat{\vartheta}_{3}$ have to satisfy the following partial differential equations

$$
\begin{aligned}
-\omega^{2} \rho \widehat{u}_{i} & =-k^{2}\left(\mu_{\mathrm{e}}+\mu_{\mathrm{c}}\right) \widehat{u}_{i} \sum_{l=1}^{2} \xi_{l}^{2}-k^{2}\left(\mu_{\mathrm{e}}-\mu_{\mathrm{c}}+\lambda_{\mathrm{e}}\right) \sum_{l=1}^{2} \widehat{u}_{l} \xi_{i} \xi_{l}-2 k \mu_{\mathrm{c}} \sum_{l=1}^{2} \varepsilon_{i l 3} \widehat{\vartheta}_{3} \xi_{l}, \quad i=1,2, \\
-\mathrm{i} \omega^{2} \rho j \mu_{\mathrm{e}} \tau_{\mathrm{c}}^{2} \widehat{\vartheta}_{3} & =-\mathrm{i} k^{2} \mu_{\mathrm{e}} L_{\mathrm{c}}^{2}\left(\alpha_{1}+\alpha_{2}\right) \sum_{l=1}^{2} \widehat{\vartheta}_{3} \xi_{l}^{2}-2 \mathrm{i} k \mu_{\mathrm{c}} \sum_{l, s=1}^{2} \varepsilon_{3 l s} \widehat{u}_{s} \xi_{l}-4 \mathrm{i} \mu_{\mathrm{c}} \widehat{\vartheta}_{3} \sum_{l=1}^{2} \xi_{l}^{2} .
\end{aligned}
$$


$w=\left(\widehat{u}_{1}, \widehat{u}_{2}, \widehat{\vartheta}_{3}\right)^{T} \neq 0$ for real positive numbers $\omega^{2}$, i.e.

$$
\left[\mathbf{Q}_{1}(\xi, k)-\omega^{2} \hat{\mathbb{1}}\right] w=0,
$$

where

$$
\mathbf{Q}_{1}(\xi, k)=\left(\begin{array}{ccc}
k^{2}\left(2 \mu_{\mathrm{e}}+\lambda_{\mathrm{e}}\right) \xi_{1}^{2}+k^{2}\left(\mu_{\mathrm{e}}+\mu_{\mathrm{c}}\right) \xi_{2}^{2} & k^{2}\left(\mu_{\mathrm{e}}-\mu_{\mathrm{c}}+\lambda_{\mathrm{e}}\right) \xi_{1} \xi_{2} & 2 k \mu_{\mathrm{c}} \xi_{2} \\
k^{2}\left(\mu_{\mathrm{e}}-\mu_{\mathrm{c}}+\lambda_{\mathrm{e}}\right) \xi_{1} \xi_{2} & k^{2}\left(\mu_{\mathrm{e}}+\mu_{\mathrm{c}}\right) \xi_{1}^{2}+k^{2}\left(2 \mu_{\mathrm{e}}+\lambda_{\mathrm{e}}\right) \xi_{2}^{2} & -2 k \mu_{\mathrm{c}} \xi_{1} \\
2 k \mu_{\mathrm{c}} \xi_{2} & -2 k \mu_{\mathrm{c}} \xi_{1} & k^{2} \mu_{\mathrm{e}} L_{\mathrm{c}}^{2}\left(\alpha_{1}+\alpha_{2}\right)\left(\xi_{1}^{2}+\xi_{2}^{2}\right)+4 \mu_{\mathrm{c}}\left(\xi_{1}^{2}+\xi_{2}^{2}\right)
\end{array}\right) .
$$

But this is equivalent to the positive definiteness of the matrix

$$
\widetilde{\mathbf{Q}}_{1}(\xi, k)=\widehat{\mathbb{1}}^{-1 / 2}\left(\begin{array}{ccc}
k^{2}\left(2 \mu_{\mathrm{e}}+\lambda_{\mathrm{e}}\right) \xi_{1}^{2}+\left(\mu_{\mathrm{e}}+\mu_{\mathrm{c}}\right) \xi_{2}^{2} & k^{2}\left(\mu_{\mathrm{e}}-\mu_{\mathrm{c}}+\lambda_{\mathrm{e}}\right) \xi_{1} \xi_{2} & 2 k \mu_{\mathrm{c}} \xi_{2} \\
k^{2}\left(\mu_{\mathrm{e}}-\mu_{\mathrm{c}}+\lambda_{\mathrm{e}}\right) \xi_{1} \xi_{2} & \left(\mu_{\mathrm{e}}+\mu_{\mathrm{c}}\right) \xi_{1}^{2}+k^{2}\left(2 \mu_{\mathrm{e}}+\lambda_{\mathrm{e}}\right) \xi_{2}^{2} & -2 k \mu_{\mathrm{c}} \xi_{1} \\
2 k \mu_{\mathrm{c}} \xi_{2} & -2 k \mu_{\mathrm{c}} \xi_{1} & k^{2}\left(\alpha_{1}+\alpha_{2}\right)+4 \mu_{\mathrm{c}}
\end{array}\right) \widehat{\mathbb{1}}^{-1 / 2} .
$$

Since the conditions (2.55) imply the positive definiteness of the matrix $\widetilde{\mathbf{Q}}_{1}(\xi, k)$ for all $\xi=\left(\xi_{1}, \xi_{2}, 0\right) \in \mathbb{R}^{3}$, $\|\xi\|=1$, taking $\xi=(\cos \theta, \sin \theta, 0)$ we deduce the desired result.

Remark 3.5. The Legendre-Hadamard ellipticity condition is not sufficient for the positive definiteness of $\mathcal{Q}_{\theta}$, since they are equivalent to (1.7) which does not imply (2.36). Instead, we will see that the Legendre-Hadamard ellipticity condition leads to the acoustic tensor and its positive definiteness. Note that there does not exist a direction $\xi \in \mathbb{R}^{3}$ such that $\mathcal{Q}_{\theta}$ equals the acoustic tensor. Moreover, even if in classical linear elasticity this is well known, in the Cosserat theory it seems to be impossible (or at least it is not obvious to us) to arrive from the Legendre-Hadamard ellipticity condition (the positive definiteness of the acoustic tensor) to the structure of the tensor $\mathcal{Q}_{\theta}$ or to its positive definiteness.

Proof. The Legendre-Hadamard ellipticity condition [2, 19,61] demands that the acoustic tensor

$$
\widehat{\mathbf{Q}}=\left(\begin{array}{cc}
\widehat{\mathbf{Q}}_{1} & 0 \\
0 & \widehat{\mathbf{Q}}_{2}
\end{array}\right)
$$

defined through

$$
\begin{aligned}
D^{2} W(\mathbf{e}, \mathfrak{K}) \cdot((\xi \otimes \eta, \zeta \otimes \eta),(\xi \otimes \eta, \zeta \otimes \eta)) & =D^{2} W_{1}(\mathbf{e}) \cdot(\xi \otimes \eta, \xi \otimes \eta)+D^{2} W_{2}(\mathfrak{K}) \cdot(\zeta \otimes \eta, \zeta \otimes \eta) \\
& =\left\langle\eta, \widehat{\mathbf{Q}}_{1}(\xi) \eta\right\rangle+\left\langle\eta, \widehat{\mathbf{Q}}_{2}(\zeta) \eta\right\rangle
\end{aligned}
$$

is strictly positive definite for any nonzero wave directions $\xi \in \mathbb{R}^{3}$ and $\zeta \in \mathbb{R}^{3}$. Hence, let us identify the matrix $\widehat{\mathbf{Q}}_{1}$ and $\widehat{\mathbf{Q}}_{2}$. First identify $\widehat{\mathbf{Q}}_{1}$. Since

$$
\begin{aligned}
D^{2} W_{1}(\mathbf{e}) .(\xi \otimes \eta, \xi \otimes \eta) & =\mu_{\mathrm{e}}\|\operatorname{sym} \xi \otimes \eta\|^{2}+\mu_{\mathrm{c}} \| \text { skew } \xi \otimes \eta \|^{2}+\frac{\lambda_{\mathrm{e}}}{2}[\operatorname{tr}(\xi \otimes \eta)]^{2} \\
& =\frac{\mu_{\mathrm{e}}+\mu_{\mathrm{c}}}{2}\|\xi\|^{2}\|\eta\|^{2}+\frac{\mu_{\mathrm{e}}-\mu_{\mathrm{c}}+\lambda_{\mathrm{e}}}{2}\langle\xi, \eta\rangle^{2} \\
& =\frac{\mu_{\mathrm{e}}+\mu_{\mathrm{c}}}{2} \sum_{i=1}^{3} \xi_{i}^{2} \sum_{i=1}^{3} \eta_{i}^{2}+\frac{\mu_{\mathrm{e}}-\mu_{\mathrm{c}}+\lambda_{\mathrm{e}}}{2}\left(\sum_{i=1}^{3} \xi_{i}^{2} \eta_{i}^{2}+2 \sum_{\substack{i, k=1 \\
i \neq k}}^{3} \xi_{i} \xi_{k} \eta_{i} \eta_{k}\right),
\end{aligned}
$$

the matrix $\widehat{\mathbf{Q}}_{1}$ having the property

$$
D^{2} W_{1}(\mathbf{e}) \cdot(\xi \otimes \eta, \xi \otimes \eta)=\left\langle\eta, \widehat{\mathbf{Q}}_{1}(\xi) \eta\right\rangle
$$

is given by

$$
\widehat{\mathbf{Q}}_{1}(\xi)=\frac{1}{2}\left(\begin{array}{ccc}
\left(2 \mu_{\mathrm{e}}+\lambda_{\mathrm{e}}\right) \xi_{1}^{2}+\left(\mu_{\mathrm{e}}+\mu_{\mathrm{c}}\right)\left(\xi_{2}^{2}+\xi_{3}^{2}\right) & \left(\mu_{\mathrm{e}}-\mu_{\mathrm{c}}+\lambda_{\mathrm{e}}\right) \xi_{1} \xi_{2} & \left(\mu_{\mathrm{e}}-\mu_{\mathrm{c}}+\lambda_{\mathrm{e}}\right) \xi_{1} \xi_{3} \\
\left(\mu_{\mathrm{e}}-\mu_{\mathrm{c}}+\lambda_{\mathrm{e}}\right) \xi_{1} \xi_{2} & \left(2 \mu_{\mathrm{e}}+\lambda_{\mathrm{e}}\right) \xi_{2}^{2}+\left(\mu_{\mathrm{e}}+\mu_{\mathrm{c}}\right)\left(\xi_{3}^{2}+\xi_{1}^{2}\right) & \left(\mu_{\mathrm{e}}-\mu_{\mathrm{c}}+\lambda_{\mathrm{e}}\right) \xi_{2} \xi_{3} \\
\left(\mu_{\mathrm{e}}-\mu_{\mathrm{c}}+\lambda_{\mathrm{e}}\right) \xi_{1} \xi_{3} & \left(\mu_{\mathrm{e}}-\mu_{\mathrm{c}}+\lambda_{\mathrm{e}}\right) \xi_{2} \xi_{3} & \left(2 \mu_{\mathrm{e}}+\lambda_{\mathrm{e}}\right) \xi_{3}^{2}+\left(\mu_{\mathrm{e}}+\mu_{\mathrm{c}}\right)\left(\xi_{1}^{2}+\xi_{2}^{2}\right)
\end{array}\right) .
$$


Next, we identify $\widehat{\mathbf{Q}}_{2}$. Since

$$
\begin{aligned}
D^{2} W_{2}(\mathfrak{K}) .(\zeta \otimes \eta, \zeta \otimes \eta) & =\alpha_{1}\|\operatorname{sym} \zeta \otimes \eta\|^{2}+\alpha_{2} \| \text { skew } \zeta \otimes \eta \|^{2}+\frac{\alpha_{3}}{2}[\operatorname{tr}(\zeta \otimes \eta)]^{2} \\
& =\frac{\alpha_{1}+\alpha_{2}}{2} \sum_{i=1}^{3} \zeta_{i}^{2} \sum_{i=1}^{3} \eta_{i}^{2}+\frac{\alpha_{1}-\alpha_{2}+\alpha_{3}}{2}\left(\sum_{i=1}^{3} \zeta_{i}^{2} \eta_{i}^{2}+2 \sum_{\substack{i, k=1 \\
i \neq k}}^{3} \zeta_{i} \zeta_{k} \eta_{i} \eta_{k}\right),
\end{aligned}
$$

the matrix $\widehat{\mathbf{Q}}_{2}$ which satisfies

$$
D^{2} W_{2}(\mathfrak{K}) \cdot(\zeta \otimes \eta, \zeta \otimes \eta)=\left\langle\eta, \widehat{\mathbf{Q}}_{2}(\zeta) \eta\right\rangle
$$

is given by

$$
\widehat{\mathbf{Q}}_{2}(\zeta)=\frac{1}{2}\left(\begin{array}{ccc}
\left(2 \alpha_{1}+\alpha_{3}\right) \zeta_{1}^{2}+\left(\alpha_{1}+\alpha_{2}\right)\left(\zeta_{1}^{2}+\zeta_{3}^{2}\right) & \left(\alpha_{1}-\alpha_{2}+\alpha_{3}\right) \zeta_{1} \zeta_{2} & \left(\alpha_{1}-\alpha_{2}+\alpha_{3}\right) \zeta_{1} \zeta_{3} \\
\left(\alpha_{1}-\alpha_{2}+\alpha_{3}\right) \zeta_{1} \zeta_{2} & \left(2 \alpha_{1}+\alpha_{3}\right) \zeta_{2}^{2}+\left(\alpha_{1}+\alpha_{2}\right)\left(\zeta_{3}^{2}+\zeta_{1}^{2}\right) & \left(\alpha_{1}-\alpha_{2}+\alpha_{3}\right) \zeta_{2} \zeta_{3} \\
\left(\alpha_{1}-\alpha_{2}+\alpha_{3}\right) \zeta_{1} \zeta_{3} & \left(\alpha_{1}-\alpha_{2}+\alpha_{3}\right) \zeta_{2} \zeta_{3} & \left(2 \alpha_{1}+\alpha_{3}\right) \zeta_{3}^{2}+\left(\alpha_{1}+\alpha_{2}\right)\left(\zeta_{1}^{2}+\zeta_{2}^{2}\right)
\end{array}\right) .
$$

Actually, our entire approach is based on the assumption that the matrix $\widetilde{\mathbf{Q}}_{1}(\xi, k)$ defined by (3.24) is positive definite. So, if the Legendre-Hadamard condition implies this fact, or it is equivalent with, then the Legendre-Hadamard condition would be also suitable for our approach. However, it seems that this is not possible because we did not find appropriate values for $\xi$ and $\zeta$ such that, for some particular values of them the positive definiteness of $\widehat{\mathbf{Q}}_{1}(\xi) \widehat{\mathbf{Q}}_{2}(\xi)$ would imply the positive definiteness of the matrix $\widetilde{\mathbf{Q}}_{1}(\xi, k)$, as we need in our approach.

Even when we consider the Legendre-Hadamard ellipticity condition on the set of admissible solutions of the form

$$
u\left(x_{1}, x_{2}, t\right)=\left(\begin{array}{c}
u_{1}\left(x_{1}, x_{2}, t\right) \\
u_{2}\left(x_{1}, x_{2}, t\right) \\
0
\end{array}\right), \quad \vartheta\left(x_{1}, x_{2}, x_{3}, t\right)=\left(\begin{array}{c}
0 \\
0 \\
\vartheta_{3}\left(x_{1}, x_{2}, t\right)
\end{array}\right),
$$

it is not clear how the positive definiteness of the matrix $\widetilde{\mathbf{Q}}_{1}(\xi, k)$ can possibly be a consequence of the LegendreHadamard ellipticity condition.

Proposition 3.6. If the constitutive coefficients satisfy the conditions (2.55), then for all $\theta \in\left(-\frac{\pi}{2}, \frac{\pi}{2}\right), k>0$ and $0 \leq v<\widehat{v}$, the tensor $\widetilde{\mathbf{Q}}_{\theta}:=\sin ^{2} \theta \boldsymbol{T}+\sin \theta \cos \theta\left(\mathcal{R}+\mathcal{R}^{T}\right)+\cos ^{2} \theta \mathcal{Q}-k^{2} v^{2} \cos ^{2} \theta \mathbb{1}$ is positive definite.

Proof. Since $\mathcal{Q}_{\theta}:=\sin ^{2} \theta \mathcal{T}+\sin \theta \cos \theta\left(\mathcal{R}+\mathcal{R}^{T}\right)+\cos ^{2} \theta \mathcal{Q}$ is positive definite, it admits only positive eigenvalues. We need to prove that for all $\theta \in\left(-\frac{\pi}{2}, \frac{\pi}{2}\right), k>0$ and for all $0 \leq v<\widehat{v}$ the matrix $\widetilde{\mathbf{Q}}_{\theta}=\mathcal{Q}_{\theta}-k^{2} v^{2} \cos ^{2} \theta \mathbb{1}$ is positive definite, which is equivalent to the property that all eigenvalues $\mathcal{Q}_{\theta}$ are larger than those of the matrix $k^{2} v^{2} \cos ^{2} \theta \mathbb{1}$, i.e., than $k^{2} v^{2} \cos ^{2} \theta$. Assuming that there exist $\theta_{0} \in\left(-\frac{\pi}{2}, \frac{\pi}{2}\right)$ and $v_{0} \in[0, \widehat{v})$ for which there is an eigenvalue $\lambda_{\theta_{0}}$ of $\mathcal{Q}_{\theta}$ such that $\lambda_{\theta_{0}}<k^{2} v_{0}^{2} \cos ^{2} \theta_{0}$, then

$$
v_{\theta_{0}}:=\sqrt{\frac{\lambda_{\theta_{0}}}{k^{2} \cos ^{2} \theta_{0}}}<v_{0}<\widehat{v}
$$

is solution of (3.21), i.e., for fixed $\theta_{0}$ we have that $v_{\theta_{0}}<\widehat{v}$ verifies

$$
\operatorname{det}\left\{\sin ^{2} \theta_{0} \mathcal{T}+\sin \theta_{0} \cos \theta_{0}\left(\mathcal{R}+\mathcal{R}^{T}\right)+\cos ^{2} \theta_{0} \mathcal{Q}-k^{2} v_{\theta_{0}}^{2} \cos ^{2} \theta_{0} \mathbb{1}\right\}=0 .
$$

This is in contradiction to the definition of the limiting speed and Proposition 3.22, since $\widehat{v}$ is the smallest speed having this property. Therefore, it remains that for all $\theta \in\left(-\frac{\pi}{2}, \frac{\pi}{2}\right), k>0$ and for all $0 \leq v<\widehat{v}$, all the eigenvalues of $\mathcal{Q}_{\theta}$ are larger than $k^{2} v^{2} \cos ^{2} \theta$ and the proof is complete.

\section{The common method to construct the solution using the Stroh formalism}

In this subsection we present the main steps of the common method using the Stroh formalism, since in this point of our approach it is still possible to switch to the Stroh formalism and vice versa, without using conceptually different methods. 

as

On one hand, using the ansatz $z\left(x_{2}\right):=\widehat{\mathbb{1}}^{1 / 2}\left(\begin{array}{l}d_{1} \\ d_{2} \\ d_{3}\end{array}\right) e^{\mathrm{i} r k x_{2}}$ in $(3.3)_{2}$, we are led to define a vector $b \neq 0 \in \mathbb{C}^{3}$

$$
b=\left[r \mathbf{T}+\mathbf{R}^{T}\right] d .
$$

Since $\mathbf{T}$ is symmetric and positive definite there exists $\mathbf{T}^{-1}$. Hence from (4.1) we get

$$
r d=-\mathbf{T}^{-1} \mathbf{R}^{T} d+\mathbf{T}^{-1} b
$$

and

$$
r b=\left[r^{2} \mathbf{T}+r \mathbf{R}^{T}\right] d .
$$

On the other hand, from $(3.11)_{1}$ and considering the same ansatz for $z\left(x_{2}\right)$ as before, we deduce

$$
\overbrace{\left(r^{2} \mathbf{T}+r \mathbf{R}^{T}\right) d}^{r b}+(r \mathbf{R}+\widetilde{\mathbf{Q}}) d=0 \quad \Leftrightarrow \quad r b=-[r \mathbf{R}+\widetilde{\mathbf{Q}}] d
$$

where $\widetilde{\mathbf{Q}}=\mathbf{Q}-k^{2} v^{2} \widehat{\mathbb{1}}$. Making use of (4.2) in (4.4) we obtain

$$
r b=-\widetilde{\mathbf{Q}} d-\mathbf{R}\left\{-\mathbf{T}^{-1} \mathbf{R}^{d} a+\mathbf{T}^{-1} b\right\}=\left[-\widetilde{\mathbf{Q}}+\mathbf{R} \mathbf{T}^{-1} \mathbf{R}^{T}\right] d-\mathbf{R} \mathbf{T}^{-1} b .
$$

From (4.2) and $(4.5)_{2}$ it is possible to indicate the relation of our analysis with the study given in [11], i.e., the scalar variable $r$ appearing in our ansatz is a solution of the following eigenvalue problem

$$
\underbrace{\left(\begin{array}{cc}
-\mathbf{T}^{-1} \mathbf{R}^{T} & \mathbf{T}^{-1} \\
\mathbf{R} \mathbf{T}^{-1} \mathbf{R}^{T}-\widetilde{\mathbf{Q}} & -\mathbf{R} \mathbf{T}^{-1}
\end{array}\right)}_{:=\mathcal{N}} \underbrace{\left(\begin{array}{l}
d \\
b
\end{array}\right)}_{:=\mathcal{V}}=r\left(\begin{array}{l}
d \\
b
\end{array}\right) \quad \Leftrightarrow \quad \mathcal{N} \mathcal{V}=r \mathcal{V}
$$

where $\mathcal{N} \in \mathbb{R}^{6 \times 6}$ is called the Stroh matrix $[67,11]$.

For Cosserat elastic materials [11] it is possible to find a suitable structure of the characteristic equation corresponding to the above eigenvalue problem in the form ${ }^{7}$

$$
r^{6}+P_{1} r^{4}+P_{2} r^{2}+P_{3}=0
$$

where

$$
\begin{aligned}
& P_{1}=C_{1}+C_{2}+C_{3}, \quad P_{2}=C_{1} C_{2}+C_{2} C_{3}+C_{3} C_{1}-\frac{4 \rho \mu_{\mathrm{c}}^{2} v^{2}}{\mu_{\mathrm{e}} L_{\mathrm{c}}^{2} \gamma\left(\mu_{\mathrm{e}}+\mu_{\mathrm{c}}\right)^{2}}, \\
& P_{3}=C_{1} C_{2} C_{3}-\frac{4 \rho \mu_{\mathrm{c}}^{2} v^{2}}{\mu_{\mathrm{e}} L_{\mathrm{c}}^{2} \gamma\left(\mu_{\mathrm{e}}+\mu_{\mathrm{c}}\right)^{2}} C_{1}, \\
& c_{l}=1-\frac{\rho v^{2}}{2 \mu_{\mathrm{e}}+\lambda_{\mathrm{e}}}, \quad c_{t}=1-\frac{\rho v^{2}}{\mu_{\mathrm{e}}+\mu_{\mathrm{c}}}, \quad c_{m}=1+\frac{4 \mu_{\mathrm{c}} \mu_{\mathrm{e}}}{\mu_{\mathrm{e}} L_{\mathrm{c}}^{2} \gamma\left(\mu_{\mathrm{e}}+\mu_{\mathrm{c}}\right)}-\frac{\rho j \mu_{\mathrm{e}} \tau_{\mathrm{c}}^{2} v^{2}}{\mu_{\mathrm{e}} L_{\mathrm{c}}^{2} \gamma} .
\end{aligned}
$$

Then, due to this structure, it is possible to factorise it as

$$
\left(r^{2}+c_{l}\right)\left[\left(r^{2}+c_{t}\right)\left(r^{2}+c_{m}\right)-\frac{4 \rho \mu_{\mathrm{c}}^{2} v^{2}}{\mu_{\mathrm{e}} L_{\mathrm{c}}^{2} \gamma\left(\mu_{\mathrm{e}}+\mu_{\mathrm{c}}\right)^{2}}\right]=0,
$$

and to find the analytical form of its solutions

$$
\begin{aligned}
& r_{1}^{2}=-c_{l}, \\
& r_{2}^{2}=\frac{1}{2}\left[-\left(c_{t}+c_{m}\right)+\sqrt{\left(c_{t}-c_{m}\right)^{2}+\frac{8 \rho \mu_{\mathrm{c}}^{2} v^{2}}{\mu_{\mathrm{e}} L_{\mathrm{c}}^{2} \gamma\left(\mu_{\mathrm{e}}+\mu_{\mathrm{c}}\right)^{2}}}\right], \\
& r_{3}^{2}=\frac{1}{2}\left[-\left(c_{t}+c_{m}\right)-\sqrt{\left(c_{t}-c_{m}\right)^{2}+\frac{8 \rho \mu_{\mathrm{c}}^{2} v^{2}}{\mu_{\mathrm{e}} L_{\mathrm{c}}^{2} \gamma\left(\mu_{\mathrm{e}}+\mu_{\mathrm{c}}\right)^{2}}}\right] .
\end{aligned}
$$

Since the explicit analytical form of the roots $r_{v}$ of the characteristic equation (4.7) as function of $v$ are known, in [11] it is shown that

\footnotetext{
${ }^{7}$ We have rewritten everything in the notation of the present paper.
} 
Proposition 4.1. If the constitutive coefficients satisfy the conditions

$$
2 \mu_{\mathrm{e}}+\lambda_{\mathrm{e}}>0, \quad \mu_{\mathrm{e}}+\mu_{\mathrm{c}}>0, \quad \alpha_{1}+\alpha_{2}>0,
$$

then the roots $r_{v}$ of the characteristic equation (4.7) are not real if and only if

$$
0 \leq v<\min \left\{\mathfrak{c}_{p}, \mathfrak{c}_{s}, \mathfrak{c}_{m_{1}}, \mathfrak{c}_{m_{2}}\right\}
$$

where

$$
\begin{aligned}
& \mathfrak{c}_{p}=\sqrt{\frac{2 \mu_{\mathrm{e}}+\lambda_{\mathrm{e}}}{\rho}}, \quad \mathfrak{c}_{m_{1}}=\sqrt{\frac{\mu_{\mathrm{e}} L_{\mathrm{c}}^{2} \gamma}{\rho j \mu_{\mathrm{e}} \tau_{\mathrm{c}}^{2}}\left[1+\frac{4 \mu_{\mathrm{c}} \mu_{\mathrm{e}}}{\mu_{\mathrm{e}} L_{\mathrm{c}}^{2} \gamma k^{2}\left(\mu_{\mathrm{e}}+\mu_{\mathrm{c}}\right)}\right]} \\
& \mathfrak{c}_{s}=\sqrt{\frac{\mu_{\mathrm{e}}+\mu_{\mathrm{c}}}{\rho}}, \quad \mathfrak{c}_{m_{2}}=\sqrt{\frac{1}{2}\left(\mathfrak{c}_{s}^{2}+\mathfrak{c}_{m_{1}}^{2}+\frac{4 \mu_{\mathrm{c}}^{2}}{k^{2} \rho j \mu_{\mathrm{e}} \tau_{\mathrm{c}}^{2}\left(\mu_{\mathrm{e}}+\mu_{\mathrm{c}}\right)}-\sqrt{\Delta}\right)} \\
& \Delta=\left(\mathfrak{c}_{s}^{2}-\mathfrak{c}_{m_{1}}^{2}\right)^{2}+2\left(\mathfrak{c}_{s}^{2}+\mathfrak{c}_{m_{1}}^{2}\right) \frac{4 \mu_{\mathrm{c}}^{2}}{k^{2} \rho j \mu_{\mathrm{e}} \tau_{\mathrm{c}}^{2}\left(\mu_{\mathrm{e}}+\mu_{\mathrm{c}}\right)}+\left(\frac{4 \mu_{\mathrm{c}}^{2}}{k^{2} \rho j \mu_{\mathrm{e}} \tau_{\mathrm{c}}^{2}\left(\mu_{\mathrm{e}}+\mu_{\mathrm{c}}\right)}\right)^{2}>0 .
\end{aligned}
$$

Remark 4.2. The inequalities (4.11) from Proposition 4.1 have the following interpretations:

i) the first two inequalities of the set of conditions (1.3) considered by Chiriţă and Ghiba [11] imply that the translational compressional wave is real and that the shear-rotational wave (optical branch) is real at high frequencies;

ii) the first inequality also implies that at the limit of high frequencies the translational compressional wave is faster than the shear-rotational wave (if they both exist);

iii) the third one means that the shear-rotational wave (acoustic branch) is real at high frequencies.

In addition,

iv) the inequalities (1.3) do not imply that the shear-rotational wave (optical branch) is real at low frequencies, i.e., $\omega \rightarrow 0$;

v) the inequalities (1.3) do not imply that the plane waves are real, i.e., that the propagating plane waves are defined only by real frequencies.

Notice that we have slightly changed the results obtained in [11], and we do not assume that $\mathfrak{c}_{p} \geq \mathfrak{c}_{s}$ because all the calculations from [11] are indeed valid without this additional assumption. Actually, the inequality $\mathfrak{c}_{p} \geq \mathfrak{c}_{s}$ holds true once the internal energy is assumed to be positive definite, i.e., when $2 \mu_{\mathrm{e}}+3 \lambda_{\mathrm{e}}>0, \mu_{\mathrm{e}}>0$. However, as we show in the present paper, the existence of the seismic waves is true for weaker conditions on the constitutive parameters.

We also mention that under the hypothesis of the above proposition, the following inequalities are valid

$$
\mathfrak{c}_{m_{2}}<\mathfrak{c}_{m_{1}}, \quad \text { and } \quad \mathfrak{c}_{m_{2}}<\mathfrak{c}_{s},
$$

since

$$
\begin{aligned}
-\sqrt{\Delta} & \leq-\left|\left(\mathfrak{c}_{s}^{2}-\mathfrak{c}_{m_{1}}^{2}\right)+\frac{4 \mu_{\mathrm{c}}^{2}}{k^{2} \rho j \mu_{\mathrm{e}} \tau_{\mathrm{c}}^{2}\left(\mu_{\mathrm{e}}+\mu_{\mathrm{c}}\right)}\right|, \\
-\sqrt{\Delta} & \leq-\left|\left(\mathfrak{c}_{m_{1}}^{2}-\mathfrak{c}_{s}^{2}\right)+\frac{4 \mu_{\mathrm{c}}^{2}}{k^{2} \rho j \mu_{\mathrm{e}} \tau_{\mathrm{c}}^{2}\left(\mu_{\mathrm{e}}+\mu_{\mathrm{c}}\right)}\right|, \\
\mathfrak{c}_{m_{2}}^{2}-\mathfrak{c}_{m_{1}}^{2} & =\frac{1}{2}\left(\mathfrak{c}_{s}^{2}-\mathfrak{c}_{m_{1}}^{2}+\frac{4 \mu_{\mathrm{c}}^{2}}{k^{2} \rho j \mu_{\mathrm{e}} \tau_{\mathrm{c}}^{2}\left(\mu_{\mathrm{e}}+\mu_{\mathrm{c}}\right)}-\sqrt{\Delta}\right)<0, \\
\mathfrak{c}_{m_{2}}^{2}-\mathfrak{c}_{s}^{2} & =\frac{1}{2}\left(\mathfrak{c}_{m_{1}}^{2}-\mathfrak{c}_{s}^{2}+\frac{4 \mu_{\mathrm{c}}^{2}}{k^{2} \rho j \mu_{\mathrm{e}} \tau_{\mathrm{c}}^{2}\left(\mu_{\mathrm{e}}+\mu_{\mathrm{c}}\right)}-\sqrt{\Delta}\right)<0 .
\end{aligned}
$$


Therefore, if the constitutive parameters satisfy the conditions 4.11 , then the speeds $\mathfrak{c}_{i}, i=1,2,3,4$ defined in (4.13), are ordered as follows

$$
\mathfrak{c}_{m_{2}}<\min \left\{\mathfrak{c}_{m_{1}}, \mathfrak{c}_{s}\right\} .
$$

This inequality does not mean that the conditions 4.11 impose (or is in contradiction with) a sort of a priori order of Eringen-type (2.40) between the speed of microscopic real waves and the speed of macroscopic real waves, since in the definition of $\boldsymbol{c}_{m_{2}}$ both the microscopic and macroscopic constitutive parameters are involved.

Using this last remark and combining Proposition 4.1 and Proposition 3.3, we conclude

Proposition 4.3. For $2 \mu_{\mathrm{e}}+\lambda_{\mathrm{e}}>0, \mu_{\mathrm{e}}>0, \mu_{\mathrm{c}}>0, \mu_{\mathrm{e}} L_{\mathrm{c}}^{2} \gamma>0$, the limiting speed in Cosserat elastic materials is given by

$$
\widehat{v}:=\inf _{\theta \in\left(-\frac{\pi}{2}, \frac{\pi}{2}\right)} v_{\theta} \equiv \min \left\{\mathfrak{c}_{p}, \mathfrak{c}_{m_{2}}\right\} .
$$

The usual Stroh algorithm to construct the solution of the seismic wave propagation problem is based on the possibility to explicitly know the analytical form of the solution $r_{v}$ of equation (4.7) as function of $v$. Then, the next step in the common methods is to let $r_{k}, k=1,2,3$ be the eigenvalues that satisfy (4.7) and the associated eigenvector are given by $\mathcal{V}^{(k)}=\left(\begin{array}{l}d^{(k)} \\ b^{(k)}\end{array}\right)$ with

$$
\begin{aligned}
& d^{(1)}=\left(\begin{array}{c}
\frac{1}{k} \\
\frac{1}{k} r_{1} \\
0
\end{array}\right), \quad d^{(2)}=\left(\begin{array}{c}
\frac{2 \mu_{\mathrm{c}}}{\left(\mu_{\mathrm{e}}+\mu_{\mathrm{c}}\right) k} r_{2} \\
-\frac{2 \mu_{\mathrm{c}}}{\left(\mu_{\mathrm{e}}+\mu_{\mathrm{c}}\right) k} \\
-\mathrm{i}\left(r_{2}^{2}+c_{t}\right)
\end{array}\right), \quad d^{(3)}=\left(\begin{array}{c}
\frac{2 \mu_{\mathrm{c}}}{\left(\mu_{\mathrm{e}}+\mu_{\mathrm{c}}\right) k} r_{3} \\
-\frac{2 \mu_{\mathrm{c}}}{\left(\mu_{\mathrm{e}}+\mu_{\mathrm{c}}\right) k} \\
-\mathrm{i}\left(r_{3}^{2}+c_{t}\right)
\end{array}\right), \\
& b^{(1)}=\left(\begin{array}{c}
2 \mu_{\mathrm{e}} r_{1} \\
\left(2 \mu_{\mathrm{e}}+\lambda_{\mathrm{e}}\right) r_{1}^{2}+\lambda_{\mathrm{e}} \\
0
\end{array}\right), \quad b^{(2)}=\left(\begin{array}{c}
\frac{2 \mu_{\mathrm{c}}}{\left(\mu_{\mathrm{e}}+\mu_{\mathrm{c}}\right)}\left[\lambda_{\mathrm{e}}+c_{l}\left(2 \mu_{\mathrm{e}}+\lambda_{\mathrm{e}}\right)\right] \\
-\frac{4 \mu_{\mathrm{c}} \mu_{\mathrm{e}}}{\left(\mu_{\mathrm{e}}+\mu_{\mathrm{c}}\right)} r_{2} \\
-\mathrm{i} k \mu_{\mathrm{e}} L_{\mathrm{c}}^{2} \gamma r_{2}\left(r_{2}^{2}+c_{t}\right)
\end{array}\right), \quad b^{(3)}=\left(\begin{array}{c}
\frac{2 \mu_{\mathrm{e}}}{\left(\mu_{\mathrm{e}}+\mu_{\mathrm{c}}\right)}\left[\lambda_{\mathrm{e}}+c_{l}\left(2 \mu_{\mathrm{e}}+\lambda_{\mathrm{e}}\right)\right] \\
-\frac{4 \mu_{\mathrm{c}} \mu_{\mathrm{e}}}{\left(\mu_{\mathrm{e}}+\mu_{\mathrm{c}}\right)} r_{3} \\
-\mathrm{i} k \mu_{\mathrm{e}} L_{\mathrm{c}}^{2} \gamma r_{3}\left(r_{3}^{2}+c_{t}\right)
\end{array}\right) .
\end{aligned}
$$

Without loss of generality, we may assume that the $r_{k}$ are distinct and, in consequence, $\mathcal{V}^{(k)}$ are linearly independent $^{8}[11]$. Then a general solution of (2.46) having a proper decay is taken in the form

$$
z=\sum_{k=1}^{3} q_{k} d^{(k)} e^{\mathrm{i} r_{k} k x_{2}}
$$

where $q_{k}$ are constants to be determined by the boundary condition $(3.3)_{2}$, i.e., we will have the system

$$
\sum_{k=1}^{3} q_{k} b^{(k)}=\mathbf{B} q=0,
$$

where

$$
\mathbf{B}=\left(b^{(1)}\left|b^{(2)}\right| b^{(3)}\right), \quad b^{(1)}=p_{k} \mathbf{T} d^{(k)}+\mathbf{R}^{T} d^{(k)}, \quad q=\left(q_{1}, q_{2}, q_{3}\right)^{T} .
$$

We have nonzero solutions $q$ if and only if

$$
\operatorname{det} \mathbf{B}=0 .
$$

We remark that the expression of $\mathbf{B}$ contains $v$, so the condition $\operatorname{det} \mathbf{B}=0$ is in fact a condition to determine $v$ and it is called the secular equation. Its explicit form $\forall v \in[0, \widehat{v})$ was determined in [11] in the following form

$$
s(v) \equiv \sqrt{P_{3}(v)\left(C_{2}(v)+C_{3}(v)+2 \sqrt{P(v)}\right)} 4 \mu_{\mathrm{e}}^{2}-\left(C_{3}(v)+\sqrt{P(v)}\right)\left[\lambda_{\mathrm{e}}-\left(2 \mu_{\mathrm{e}}+\lambda_{\mathrm{e}}\right) C_{1}(v)\right]^{2}=0,
$$

where

$$
P(v)=P_{2}(v)-C_{1}(v) C_{2}(v)-C_{1}(v) C_{3}(v)
$$

and the notations (4.8) are used. In [11, Eq. (4.14)] the following result is established

\footnotetext{
${ }^{8}$ If $r_{k}$ are not distinct, then we would find $d^{(k)}$ eigenvectors associated to $r_{k}$ which are independent.
} 
Theorem 4.4. If the constitutive coefficients satisfy

$$
\mu_{\mathrm{e}}-\mu_{\mathrm{c}}+\lambda_{\mathrm{e}}>0, \quad \mu_{\mathrm{e}}+\mu_{\mathrm{c}}>0, \quad \alpha_{1}+\alpha_{2}>0 \quad \text { (these conditions imply } 2 \mu_{\mathrm{e}}+\lambda_{\mathrm{e}}>0 \text { ) }
$$

then, the secular equation (4.22) has an admissible solution, i.e. a solution $v$ such that $0 \leq v<\widehat{v}$.

\section{Remark 4.5.}

- The very important aspect in analysing the secular equation is to prove that it has an admissible solution, i.e., there exists at least one solution $v$ such that $0 \leq v<\widehat{v}$, since otherwise the constructed wave ansatz does not satisfies the asymptotic decay condition (2.46). This is the essential condition in modelling seismic waves and it will validate or not the entire approach used for the construction of the solution.

- As always in the modelling process, the uniqueness of the desired solution is also a very important aspect, because if the uniqueness is not clearly stated then the question of which solution has to be effectively chosen arises.

However, in almost all studies concerning the propagation of seismic waves in generalized theories of solid mechanics, the complete study of the existence and uniqueness problem of an admissible solution of the corresponding secular equation is often left unsolved. This is also true for the case of the linear Cosserat theory. This question is completely settled by the present paper.

\section{The new secular equation. Existence and uniqueness of Rayleigh waves}

\subsection{Derivation and matrix analysis of the algebraic Riccati equation}

The main ingredient of the method used by Fu and Mielke [24] is to look at (3.8) as an initial value problem and to search for a solution in the form

$$
y\left(x_{2}\right)=e^{-k x_{2} \mathcal{E}} y(0),
$$

where $\mathcal{E} \in \mathbb{C}^{3 \times 3}$ is to be determined. On substituting (5.1) into (3.12), we get

$$
\left[\mathcal{T} \mathcal{E}^{2}-\mathrm{i}\left(\mathcal{R}+\mathcal{R}^{T}\right) \mathcal{E}-\mathcal{Q}+k^{2} v^{2} \mathbb{1}\right] y\left(x_{2}\right)=0, \quad\left(-\mathcal{T} \mathcal{E}+\mathrm{i} \mathcal{R}^{T}\right) y(0)=0 .
$$

It is clear that in order to have a proper decay the eigenvalues of $\mathcal{E}$ have to be such that their real part is positive. We anticipate and we mention that this will be the case if $0 \leq v<\widehat{v}$, as we will see in Theorem 5.2.

For the linear Cosserat model, we introduce the so called surface impedance matrix

$$
\mathcal{M}=-\left(-\mathcal{T} \mathcal{E}+\mathrm{i} \mathcal{R}^{T}\right) \quad \Longleftrightarrow \quad \mathcal{E}=\mathcal{T}^{-1}\left(\mathcal{M}+\mathrm{i} \mathcal{R}^{T}\right)
$$

It seems that this matrix was first introduced by Ingebrigsten and Tonning [31] for the classical elastic model, by Mielke and Sprenger [48] on a topic indirectly connected to the surface wave problem, and then by $\mathrm{Fu}$ and Mielke $[47,24]$ in order to prove the existence and the uniqueness of the surface-wave speed for linear anisotropic elastic materials. We may argue the utility of this replacement of $\mathcal{E}$ to $\mathcal{M}$ in order to convert the equation $(5.2)_{2}$ into an equation for a Hermitian matrix $\mathcal{M}$. On substituting $(5.3)_{2}$ into $(5.2)_{1}$, we obtain

$$
\begin{array}{r}
\left\{\mathcal{T} \mathcal{E}-\mathrm{i}\left(\mathcal{R}+\mathcal{R}^{T}\right)\right\} \mathcal{E}-\mathcal{Q}+k^{2} v^{2} \mathbb{1}=0, \\
\left(\mathcal{M}+\mathrm{i} \mathcal{R}^{T}\right) \mathcal{T}^{-1}\left(\mathcal{M}+\mathrm{i} \mathcal{R}^{T}\right)-\mathrm{i} \mathcal{R} \mathcal{T}^{-1}\left(\mathcal{M}+\mathrm{i} \mathcal{R}^{T}\right)-\mathrm{i} \mathcal{R}^{T} \mathcal{T}^{-1}\left(\mathcal{M}+\mathrm{i} \mathcal{R}^{T}\right)-\mathcal{Q}+k^{2} v^{2} \mathbb{1}=0, \\
\mathcal{M} \mathcal{T}^{-1}\left(\mathcal{M}+\mathrm{i} \mathcal{R}^{T}\right)-\mathrm{i} \mathcal{R} \mathcal{T}^{-1}\left(\mathcal{M}+\mathrm{i} \mathcal{R}^{T}\right)-\mathcal{Q}+k^{2} v^{2} \mathbb{1}=0
\end{array}
$$

Hence, in terms of the surface impedance matrix, equations (5.2) become

$$
(\mathcal{M}-\mathrm{i} \mathcal{R}) \mathcal{T}^{-1}\left(\mathcal{M}+\mathrm{i} \mathcal{R}^{\mathcal{T}}\right)-\mathcal{Q}+k v^{2} \mathbb{1}=0, \quad \mathcal{M} y(0)=0 .
$$

We call equation (5.5) 1 algebraic Riccati equation for the linear Cosserat model. For the classical anisotropic model, the same form of this equation is present in the paper by Mielke and Sprenger [48] for $v=0$ 
and in the papers by Fu and Mielke $[47,24]$ in the case of general $v$, see also the earlier work by Biryukov [7]. For this reason, our expectation is that the entire approach presented by Fu and Mielke [47, 24] should be suitable in the Cosserat theory, too.

Since we are interested in a nontrivial solution $y$, we impose $y(0) \neq 0$, so that the matrix $\mathcal{M}$ has to satisfy

$$
\operatorname{det} \mathcal{M}=0 .
$$

The equation (5.6) is called secular equation for the linear Cosserat model in terms of the impedance matrix $\mathcal{M}$.

In this point of our analysis we do not have additional informations about the properties of the impedance matrix $\mathcal{M}$. Looking at the equations (5.5) and (5.6) we remark that equation $(5.5)_{1}$ leads to a mapping $v \mapsto \mathcal{M}_{v}$, where $\mathcal{M}_{v}$ is the solution of $(5.5)_{1}$ for a fixed $v$. If it is possible to construct this mapping, then equation (5.6) becomes the equation which determines the wave speed $v$. However, the construction of this mapping is possible only if we are very careful with the following aspects. The first yet unresolved problem is the knowledge of the domain of those $v$ for which $(5.5)_{1}$ admits a unique solution. The second question is: does the solution of the other equation (5.6), with $\mathcal{M}_{v}$ expressed as function of $v$, belong (if it exists) to the domains of those $v$ for which (5.5) 1 admits a solution? The last but not the least important aspect is that it is not sufficient to prove that there is a solution $\mathcal{M}_{v}$ of the Riccati equation (5.5), since an acceptable $\mathcal{M}_{v}$ has to be such that $\operatorname{Re}(\operatorname{spec} \mathcal{E})$ is positive, where "Re $(\operatorname{spec} \mathcal{E})$ " means the "real part of spectra of $\mathcal{E}$ ".

Similar arguments as for the classical linear anisotropic elastic materials lead us to the following

Lemma 5.1. If the constitutive coefficients satisfy the conditions (2.55) and $0 \leq v<\widehat{v}$, then the eigenvalues of any solution of (5.5) have a non-zero real part.

Proof. Let $\mathcal{E}$ be a solution of (5.5), $\lambda$ be an eigenvalue and $a$ an associated eigenvector, i.e. $\mathcal{E} d=\lambda d$. Hence, if $\mathcal{E}$ is a solution of (5.5), then

$$
\left\{\lambda^{2} \mathcal{T}-\mathrm{i} \lambda\left(\mathcal{R}+\mathcal{R}^{T}\right) \mathcal{E}-\mathcal{Q}+k^{2} v^{2} \mathbb{1}\right\} d=0,
$$

and $\lambda$ is a solution of the equation

$$
\operatorname{det}\left\{\lambda^{2} \mathcal{T}-\mathrm{i} \lambda\left(\mathcal{R}+\mathcal{R}^{T}\right) \mathcal{E}-\mathcal{Q}+k^{2} v^{2} \mathbb{1}\right\}=0 .
$$

Thus, $r=\mathrm{i} \lambda$ is solution of the equation

$$
\operatorname{det}\left\{-r^{2} \mathcal{T}-r\left(\mathcal{R}+\mathcal{R}^{T}\right) \mathcal{E}-\mathcal{Q}+k^{2} v^{2} \mathbb{1}\right\}=0 \quad \Leftrightarrow \quad \operatorname{det}\left\{r^{2} \mathcal{T}+r\left(\mathcal{R}+\mathcal{R}^{T}\right) \mathcal{E}+\mathcal{Q}-k^{2} v^{2} \mathbb{1}\right\}=0 .
$$

Assuming that there exists an eigenvalue $\lambda$ of a solution $\mathcal{E}$ of (5.5) with a non-zero real part, it follows that the equation

$$
\operatorname{det}\left\{r^{2} \mathcal{T}+r\left(\mathcal{R}+\mathcal{R}^{T}\right) \mathcal{E}+\mathcal{Q}-k^{2} v^{2} \mathbb{1}\right\}=0
$$

admits a non-real solution. But we have shown in Proposition 3.3 that this is not possible if the wave speed is smaller than the limiting speed $\widehat{v}$.

Under the assumption that $\mathcal{T}$ and $\mathcal{Q}$ are symmetric and positive definite matrices, many aspects from the above discussions are purely mathematical questions, and they are not specific to Cosserat elastic materials. Notice that we were careful to obtain a specific formulation such that many mathematical results obtained by Mielke and $\mathrm{Fu}[47,24]$ can be directly applied in the Cosserat theory, too. For instance, since $\mathcal{T}, \mathcal{Q}$ and $\widehat{\mathbb{1}}$ are symmetric real matrices, the following result established in $[47,24]$ remains valid in the framework of the Cosserat elastic materials

Theorem 5.2. If the constitutive coefficients satisfy the conditions (2.55) and $0 \leq v<\widehat{v}$, the matrix problem

$$
\mathcal{T} \mathcal{E}^{2}-\mathrm{i}\left(\mathcal{R}+\mathcal{R}^{T}\right) \mathcal{E}-\mathcal{Q}+k^{2} v^{2} \mathbb{1}=0, \quad \operatorname{Re}(\operatorname{spec} \mathcal{E})>0,
$$

has a unique solution for $\mathcal{E}_{v}$ and the corresponding matrix $\mathcal{M}_{v}$ obtained from $(5.3)_{2}$ is Hermitian.

Proof. The reader may consult the paper by Fu and Mielke [24] or the Appendix, where we have rewritten the proof in our notation. 
Hence, Theorem 5.2 proves that for all $0 \leq v<\widehat{v}$ there exists a unique solution $\mathcal{M}_{v}$ of the Riccati equation (5.5), defined by the unique matrix $\mathcal{E}_{v}$ indicated in Theorem 5.2.

Thus, we know that we can consider the mapping which associates to each $0 \leq v<\widehat{v}$ the Hermitian matrix $\mathcal{M}_{v}$ satisfying the equation (5.5). However, the pair $\left(v, \mathcal{M}_{v}\right)$ must also be a solution of the secular equation (5.6), i.e.,

$$
\operatorname{det} \mathcal{M}_{v}=0
$$

Therefore, we have to check if after replacing the solution $\mathcal{M}_{v}$ of (5.5) into (5.12), the resulting equation will lead to a unique wave speed belonging to the interval $[0, \widehat{v})$. Without explaining this aspect and having a clear answer, the analyses would be incomplete.

In the context of linear anisotropic elasticity Barnett and Lothe [5] have proven that the secular equation has a unique subsonic solution and their proof is based on the following properties of the impedance matrix $\mathcal{M}_{v}$ from the classical linear elastic model, that hold for a subsonic solution $v$ :

1. The surface impedance matrix $\mathcal{M}_{v}$ is Hermitian,

2. The matrix $\frac{d \mathcal{M}_{v}}{d v}$ is negative definite,

3. $\left\langle\mathcal{M}_{v}, \mathbb{1}\right\rangle \geq 0$, and $\left\langle w, \mathcal{M}_{v} w\right\rangle \geq 0$ for all real vectors $w$.

In the following we show that for subsonic wave speeds $v$, the impedance matrix $\boldsymbol{M}_{v}$, solution of (5.5), satisfies the above properties 1-3 for isotropic elastic Cosserat materials, too. It is straightforward to prove the second property using the same arguments as in the proof given by $\mathrm{Fu}$ and Mielke [24] in the context of anisotropic classical elastic materials, since the main problem is in fact a purely mathematical question, independent of the considered theory. Indeed, the proof remains unchanged in the context of isotropic elastic Cosserat materials considered in our paper

Theorem 5.3. Assume the constitutive coefficients satisfy the conditions (2.55) and $0 \leq v<\widehat{v}$. Let $\mathcal{M}_{v}$ and $\mathcal{E}_{v}$ be the same as in the conclusion of Theorem 5.2. Then the matrix $\frac{d \mathcal{M}_{v}}{d v}$ is negative definite.

Proof. The reader may consult the paper by Fu and Mielke [24] or the Appendix, where we have rewritten the proof in our notations.

In the following, we prove that the impedance matrix $\boldsymbol{M}_{v}$ satisfies also the 3rd property. To this aim we follow again Fu and Mielke's technique [24] and we show that this method is also applicable to the linear isotropic elastic Cosserat model. Hence, in order to establish the 3 rd property, we first define matrices $\widetilde{\mathcal{Q}}_{\theta}, \mathcal{T}_{\theta}$ and $\mathcal{R}_{\theta} \in \mathbb{R}^{3 \times 3}$ by

$$
\left[\begin{array}{cc}
\widetilde{\mathcal{Q}}_{\theta} & \mathcal{R}_{\theta} \\
\mathcal{R}_{\theta}^{\mathbf{T}} & \mathcal{T}_{\theta}
\end{array}\right]=\left[\begin{array}{cc}
\cos \theta \mathbb{1} & \sin \theta \mathbb{1} \\
-\sin \theta \mathbb{1} & \cos \theta \mathbb{1}
\end{array}\right]\left[\begin{array}{cc}
\widetilde{\mathcal{Q}} & \mathcal{R} \\
\mathcal{R}^{\mathbf{T}} & \mathcal{T}
\end{array}\right]\left[\begin{array}{cc}
\cos \theta \mathbb{1} & -\sin \theta \mathbb{1} \\
\sin \theta \mathbb{1} & \cos \theta \mathbb{1}
\end{array}\right],
$$

where we write $\widetilde{\mathcal{Q}}=\mathcal{Q}-k v^{2} \mathbb{1}$, and $\theta$ is an arbitrary angle. These matrices may be seen as the counterpart of $\mathcal{T}, \widetilde{\mathcal{Q}}$ and $\mathcal{R}$ and they are obtained by rotation of the old coordinate system about $e_{3}$ by an angle $\theta$

$$
\begin{aligned}
\mathcal{T}_{\theta} & =\cos ^{2} \theta \mathcal{T}-\sin \theta \cos \theta\left(\mathcal{R}+\mathcal{R}^{T}\right)+\sin ^{2} \theta \widetilde{\mathcal{Q}} \\
\mathcal{R}_{\theta} & =\cos ^{2} \theta \mathcal{R}+\sin \theta \cos \theta(\mathcal{T}-\widetilde{\mathcal{Q}})-\sin ^{2} \theta \mathcal{R}^{T}, \\
\widetilde{\mathcal{Q}}_{\theta} & =\cos ^{2} \theta \widetilde{\mathcal{Q}}+\sin \theta \cos \theta\left(\mathcal{R}+\mathcal{R}^{T}\right)+\sin ^{2} \theta \mathcal{T}
\end{aligned}
$$

The new matrices $\mathcal{T}$ and $\mathcal{Q}$ remain symmetric and $\widetilde{\mathcal{Q}}_{\theta}, \mathcal{T}_{\theta}$ and $\boldsymbol{\mathcal { R }}_{\theta}$ are periodic in $\theta$ with periodicity $\pi$ and

$$
\widetilde{\mathcal{Q}}_{\theta}\left(\theta+\frac{\pi}{2}\right)=\mathcal{T}_{\theta}, \quad \mathcal{R}_{\theta}\left(\theta+\frac{\pi}{2}\right)=-\mathcal{R}_{\theta}^{T}, \quad \mathcal{T}_{\theta}\left(\theta+\frac{\pi}{2}\right)=\widetilde{\mathcal{Q}}_{\theta}
$$

Moreover, according to the definition of the limiting speed, regarding Proposition 3.3 and Proposition 3.6, the limiting velocity $\widehat{v}$ is in fact the lowest velocity for which the matrices $\widetilde{\mathcal{Q}}_{\theta}$ and $T(\theta)$ become singular for some angle $\theta$ and $\widetilde{\mathcal{Q}}_{\theta}$ is positive definite for $0 \leq v<\widehat{v}$. In view of (5.15) so is $\mathcal{T}_{\theta}$, too. Thus by the definition of the 
limiting speed $\widehat{v}$ both $\widetilde{\mathcal{Q}}_{\theta}$ and $\mathcal{T}_{\theta}$ are positive definite or positive semi-definite depending on $\theta$ (for $v=\widehat{v}$ there is at least one $\theta$ at which $\mathcal{T}(\theta)$ has an eigenvalue 0 , and likewise $\widetilde{\mathcal{Q}}_{\theta}$ ).

We shall use $\mathcal{E}$ exclusively to denote the unique solution of (5.2), and likewise we define $\boldsymbol{E}_{\theta}$ to be the unique solution of the matrix problem ${ }^{9}$

$$
\mathcal{T}_{\theta} \mathcal{E}_{\theta}^{2}-\mathrm{i}\left(\mathcal{R}_{\theta}+\mathcal{R}_{\theta}^{T}\right) \mathcal{E}_{\theta}-\widetilde{\mathcal{Q}}_{\theta}=0 \quad \text { Re spec } \mathcal{E}_{\theta}>0,
$$

while the matrix $\mathcal{M}_{\theta}$ has a form such that

$$
\mathcal{E}_{\theta}=\mathcal{T}_{\theta}^{-1}\left(\mathcal{M}_{\theta}+\mathrm{i} \mathcal{R}_{\theta}^{T}\right)
$$

In terms of the matrix $\mathcal{M}_{\theta}$ the equation (5.16) reads

$$
\left(\mathcal{M}_{\theta}-\mathrm{i} \mathcal{R}_{\theta}\right) \mathcal{T}_{\theta}^{-1}\left(\mathcal{M}_{\theta}+\mathrm{i} \mathcal{R}_{\theta}^{T}\right)-\mathcal{Q}_{\theta}^{T}=0 .
$$

The following results established in [24] remain valid in our framework, too.

Theorem 5.4. Assume the constitutive coefficients satisfy the conditions (2.55) and $0 \leq v<\widehat{v}$. Then,

i) The Hermitian matrix $\mathcal{M}_{\theta}$ defined above is independent of $\theta$.

ii) Denoting by $\mathcal{M}$ and $\mathcal{E}$ the corresponding values of $\mathcal{M}_{\theta}$, and $\mathcal{E}_{\theta}$ for $\theta=0$, respectively, then $\mathcal{E}_{\theta}=(\cos \theta \mathbb{1}+\mathrm{i} \sin \theta \mathcal{E})^{-1}(\cos \theta \mathcal{E}+\mathrm{i} \sin \theta \mathbb{1})$.

iii) $\int_{0}^{\pi} \mathcal{E}_{\theta} d \theta=\pi \mathbb{1}$

iv) The unique solution of the algebraic Riccati equation (5.5) that satisfies $\operatorname{Re}\left[\operatorname{spec}\left(\mathcal{T}^{-1}\left(\mathcal{M}+\mathrm{i} R^{T}\right)\right)\right]>0$ is given explicitly by

$$
\mathcal{M}_{v}=\mathbf{H}_{v}^{-1}+\mathrm{i} \mathbf{H}_{v}^{-1} \mathbf{S}_{v}, \quad \text { with } \quad \mathbf{H}_{v}=\frac{1}{\pi} \int_{0}^{\pi} \mathcal{T}_{\theta}^{-1} d \theta, \quad \mathbf{S}_{v}=-\frac{1}{\pi} \int_{0}^{\pi} \mathcal{T}_{\theta}^{-1} \mathcal{R}_{\theta}^{T} d \theta .
$$

Proof. The reader may consult the paper by Fu and Mielke [24] or the Appendix, where we have rewritten the proof in our notations.

From a computational point of view, the decisive advantage given by the above result is that, since $\boldsymbol{T}_{\theta}$ and $\boldsymbol{\mathcal { R }}_{\theta}$ depend on the wave speed $v$, we obtain the explicit form of the secular equation $\operatorname{det} \boldsymbol{\mathcal { M }}_{v}=0$ without a priori knowing the analytical expressions (as function of the wave speed ) of the eigenvalues that satisfy (3.13) and the associated eigenvector $d^{(k)}$, which is the main difficulty in almost all the generalised models, with exception of some models for which this task is straightforward, e.g., classical isotropic linear elasticity [1, 30], or the theory of materials with voids $[57,10,62]$ after imposing restrictive conditions upon the constitutive coefficients. After the secular equation is solved, the task of finding the eigenvalues that satisfy (3.13) and the associated eigenvector $d^{(k)}$ becomes a purely numerical task, avoiding symbolic (analytical) computations.

\subsection{The main result: Existence and uniqueness of Rayleigh waves}

Moreover, Fu and Mielke's method has another advantage since we are able to show the existence and uniqueness of a subsonic wave speed, solution of the secular equation, which ensures in the end that there exists an acceptable $\mathcal{M}_{v}$ such that $\operatorname{Re} \operatorname{spec} \mathcal{E}$ is positive, i.e. the solution satisfies both the boundary conditions (2.45) and the decay conditions (2.46). As we will explain in the following, the matrix $\mathcal{M}_{v}$ determined by (5.19) satisfies the condition 3., i.e. $\operatorname{tr}\left(\mathcal{M}_{v}\right) \geq 0$, and $\left\langle w, \mathcal{M}_{v} w\right\rangle \geq 0$ for all real vectors $w$, and this will imply the existence of a unique subsonic solution of the secular equation.

\footnotetext{
${ }^{9}$ This solution exists since $\boldsymbol{\mathcal { T }}_{\theta}$ and $\widetilde{\mathcal{Q}}_{\theta}$ are positive definite. Then, Theorem 5.2 will be used since it is valid for $v=0$, too.
} 
Theorem 5.5. [The main result of this paper] Assume the constitutive coefficients satisfy the conditions

$$
2 \mu_{\mathrm{e}}+\lambda_{\mathrm{e}}>0, \quad \mu_{\mathrm{e}}>0, \quad \mu_{\mathrm{c}}>0, \quad \alpha_{1}+\alpha_{2}>0,
$$

then the secular equation

$$
\operatorname{det} \mathcal{M}_{v}=0
$$

where $\mathcal{M}_{v}$ is given by (5.19), has a unique admissible solution $0 \leq v<\widehat{v}$. In other words there exists a unique Rayleigh wave propagating in the Cosserat medium.

Proof. First, we explain why, if $\mathcal{E}_{v}$ solves (5.11), then the corresponding $\mathcal{M}_{v}$ obtained from $(5.3)_{2}$ has the following properties

1. $\mathcal{M}_{v}$ is Hermitian,

2. $\frac{d \mathcal{M}_{v}}{d v}$ is negative definite,

3. $\operatorname{tr}\left(\mathcal{M}_{v}\right) \geq 0$, and $\left\langle w, \mathcal{M}_{v} w\right\rangle \geq 0$ for all real vectors $w$ for all $0 \leq v \leq \widehat{v}$,

4. $\mathcal{M}_{v}$ is and positive definite for all $0 \leq v<\widehat{v}$.

To this aim, we can use the arguments explained in [47, page 13]. Since due to Theorem 5.2 we know that $\mathcal{M}_{v}$ is Hermitian, $\mathbf{H}_{v}$ and $\mathbf{S}_{v}$ are both real matrices and $\mathbf{H}_{v}$ is symmetric, it follows that $\mathbf{H}_{v}^{-1} \mathbf{S}_{v}$ is skew-symmetric. Hence, $\operatorname{tr}\left(\mathcal{M}_{v}\right)=\operatorname{tr}\left(\mathbf{H}_{v}^{-1}\right)$ and $\left\langle\mathcal{M}_{v} w, w\right\rangle=\left\langle\mathbf{H}_{v}^{-1} w, w\right\rangle \forall w \in \mathbb{R}^{3}$, since $\mathbf{H}_{v}^{-1} \mathbf{S}_{v}$ is skew-symmetric. Since $\mathbf{H}_{v}^{-1}$ is positive semi-definite ${ }^{10}$ for all $0 \leq v \leq \widehat{v}$ and positive definite for all $0 \leq v<\widehat{v}$, it follows that $\boldsymbol{M}_{v}$ determined by (5.19) satisfies the condition 3 and moreover $\mathcal{M}_{v}$ is positive definite for all $0 \leq v<\widehat{v}$. Note that at $v=\widehat{v}$ at least one of the eigenvalues of $\mathbf{H}_{v}^{-1}$ must vanish.

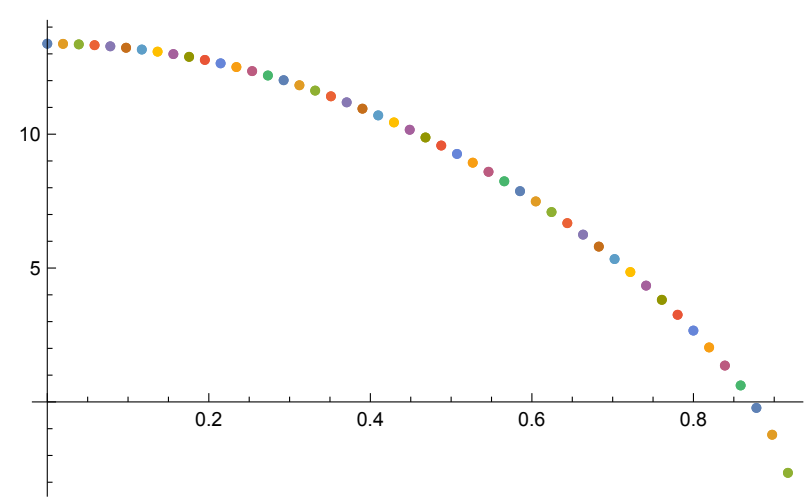

Figure 2: A plot of $\operatorname{det} \mathcal{M}_{v}$ with respect to the surface waves speed $v$ for the aluminum-epoxy composite for a set of equidistant values in the interval $[0, \widehat{v})$. This curve illustrates that $\operatorname{det} \mathcal{M}_{v}$ is a decreasing function of the wave speed $v$.

The rest of the proof is clearly explained in [24, page 2531] as in the following. Since $\frac{d \mathcal{M}_{v}}{d v}$ is negative definite, the eigenvalues of $\boldsymbol{\mathcal { M }}_{v}$ are monotone decreasing functions of $v$ defined on $[0, \widehat{v})$. Let us remark that at $v=0$ the eigenvalues of $\mathcal{M}_{0}$ are positive, since $\mathcal{M}_{v}$ is positive definite at $v=0$, that $\operatorname{det} \mathcal{M}_{v}=\lambda_{1} \lambda_{2} \lambda_{3}$, where $\lambda_{1}, \lambda_{2}, \lambda_{3}$ denote the eigenvalues of $\mathcal{M}_{v}$, that $\operatorname{det} \mathcal{M}_{0}>0$ and that the map $v \mapsto \operatorname{det} \mathcal{M}_{v}$ is monotone decreasing on $[0, \widehat{v})$, too. Thus, there exists a solution of the secular equation $\operatorname{det} \boldsymbol{\mathcal { M }}_{v}=0$ only if an eigenvalue of $\boldsymbol{M}_{v}$ decreases at zero at $0<v=v_{R}<\widehat{v}$. Moreover, if such a $v_{R}$ exists it is unique, in the sense that only one eigenvalues of $\mathcal{M}_{v}$ may decrease to zero for a value $0<v=v_{R}<\widehat{v}$ of the wave speed, since if two eigenvalues would share this property, then at $v=\widehat{v}$ the matrix $\mathcal{M}_{v}$ should have two ${ }^{11}$ eigenvalues which are

\footnotetext{
${ }^{10}$ Note that $\mathbf{H}_{v}$ is not well defined for $v=\widehat{v}$ since for this value of the wave speed there exists an angle $\theta \in[0, \pi]$ such that $\mathcal{T}_{\theta}$ has a zero eigenvalue. However, $\mathbf{H}_{v}^{-1}$ is well-defined for the limit $v \rightarrow \widehat{v}$ and at this limit it admits a zero eigenvalue, since considering the contrary it follows that $\mathbf{H}_{v}$ is well-defined for the limit $v \rightarrow \widehat{v}$. This will imply that $\boldsymbol{\mathcal { T }}_{\theta}^{-1}$ is defined for the limit $v \rightarrow \widehat{v}$ and that $\mathcal{T}_{\theta}$ and $\mathcal{Q}_{\theta}$ are positive definite for the limit $v \rightarrow \widehat{v}$, too, a fact that is contrary to the property of the limiting speed.

${ }^{11}$ There cannot exist three negative eigenvalues $\operatorname{since} \operatorname{tr}\left(\mathcal{M}_{v}\right) \geq 0$.
} 
negative which will violate the positive semi-definiteness ${ }^{12}$ of $\mathbf{H}_{v}^{-1}$ at $v=\widehat{v}$ since at least one eigenvalue of $\mathbf{H}_{v}^{-1}$ at $v=\widehat{v}$ is zero. In the same manner we argue that at $v=v_{R}$ zero there is not a repeated eigenvalue of $\boldsymbol{M}_{v}$. We conclude the proof by pointing out that there exists a unique $v_{R}$ such that an eigenvalue of $\boldsymbol{\mathcal { M }}_{v}$ decreases to zero at $0<v=v_{R}<\widehat{v}$, and therefore that $\operatorname{det} \boldsymbol{M}_{v_{R}}=0$, while $\operatorname{det} \boldsymbol{\mathcal { M }}_{v}>0$ for all $0<v<v_{R}$ and $\operatorname{det} \mathcal{M}_{v}<0$ for all $v_{R}<v<\widehat{v}$.

Here, we also illustrate the statements from the above paragraph numerically for the aluminum-epoxy composite considered by Gauthier [26] and Eringen [20]. Since the integral representation (5.19) is an explicit expression for the surface impedance matrix, we simply increase $v$ in small steps from 0 to the determined $\widehat{v}$ at every step. Plotting the secular equation $\operatorname{det} \mathcal{M}_{v}$ with respect to the wave speed $v$ gives the curve shown in Figure 2. We observe how simple it is to find an approximation of the wave speed, once the integral representation (5.19) is given.

Comparing with the left hand side of the secular equation presented in [11, Eq. (4.7)] the left hand side of our new secular equation involve a strictly decreasing function, see Figure 2 and [11, Fig. 1]. Moreover, we have analytically proven that the left hand side defines a decreasing function for all materials.

In our analysis we have considered $L_{\mathrm{c}}>0$. Thus, the results are valid for the full Cosserat medium. Part of our results and estimates may be immediately applicable to the case of the reduced Cosserat model (considered in $[27,35])$ by simply taking $L_{\mathrm{c}} \rightarrow 0$. However, since we do not know explicitly how the analytical form of the solution $v$ of the secular equation depends on $L_{\mathrm{c}}$, we are not able to obtain the form of the solution $v$ for the reduced Cosserat model by simply letting $L_{\mathrm{c}} \rightarrow 0$ in the expression of the solution $v$ for the full Cosserat model. But, all our calculations may be adapted to the case $L_{\mathrm{c}}=0$.

\section{Numerical implementation}

In this section we consider $k=1 \mathrm{~mm}^{-1}$ and the constitutive coefficients obtained by Gauthier [26], see also [20], for aluminum-epoxy composite. According to [20, pages 164-165], in the Eringen notations [28], for such a material we have

$$
\begin{aligned}
& \lambda_{\text {Eringen }}=7.59 \mathrm{GPa}, \quad \quad \mu_{\text {Eringen }}=1.89 \mathrm{GPa}, \quad \kappa_{\text {Eringen }}=0.00788 \mu_{\text {Eringen }}, \\
& \rho=\frac{\kappa_{\text {Eringen }}}{0.0067}, \quad j_{\text {Eringen }}=0.0196 \mathrm{~mm}^{2}, \quad \gamma_{\text {Eringen }}=7.11 j_{\text {Eringen }} \mu_{\text {Eringen }},
\end{aligned}
$$

while in our notation the same onstitutive coefficients are represented by

$$
\begin{aligned}
& \lambda_{\mathrm{e}}=7.59 \mathrm{GPa}, \quad \quad \mu_{\mathrm{c}}=\frac{\kappa}{2}=0.0074466 \mathrm{GPa}, \quad \quad \mu_{\mathrm{e}}=\mu_{\text {Eringen }}+\frac{\kappa}{2}=1.89745 \mathrm{GPa}, \\
& \rho=2.22287 \frac{g}{\mathrm{~mm}^{3}}, \quad j \mu_{\mathrm{e}} \tau_{\mathrm{c}}^{2}=0.0196 \mathrm{~mm}^{2}, \quad \quad \mu_{\mathrm{e}} L_{\mathrm{c}}^{2} \gamma=0.263383 \mathrm{GPa} \times \mathrm{mm} .
\end{aligned}
$$

According to the results presented in the previous sections, for known numerical values of all constitutive parameters, we identify an algorithm to approximate numerically the problem of the propagation of seismic waves:

\section{A first algorithm:}

Step 1: Identify the limiting speed $\widehat{v}$. Since the constitutive coefficients satisfy (4.11), using Proposition 4.1 and after direct substitution in its analytical form, we find the value of the limiting speed $\widehat{\boldsymbol{v}}=\mathbf{0 . 9 2 5 5 0 7}$. Let us notice that it defers from that established in [11], because after reverification we have remarked that all the numerical computations in [11] are done for other values of the constitutive parameters, which do not match those proposed by Gauthier [26] for aluminum-epoxy composite. Due to a common misunderstanding of the notations, in [11] it is considered that $\mu_{\mathrm{e}} L_{\mathrm{c}}^{2} \gamma=5.8546$ and $J=\rho j \mu_{\mathrm{e}} \tau_{\mathrm{c}}^{2}=0.4357$. However, the numerical calculation given in [11] are correct, but for the before mentioned values of $\mu_{\mathrm{e}} L_{\mathrm{c}}^{2} \gamma$ and $J$. We have repeated the calculations in the sense of the approach given in [11] and we have found a complete agreement with the value of the limiting speed considered in the current paper, i.e., $\widehat{\boldsymbol{v}}=\mathbf{0 . 9 2 5 5 0 7}$.

\footnotetext{
${ }^{12}$ We recall that the positive semi-definiteness of $\mathbf{H}_{v}^{-1}$ is equivalent to the positive semi-definiteness of $\mathcal{M}_{v}$.
} 
Step 2: Find the solution of the secular equation. We have applied formula (5.19) to compute $\boldsymbol{\mathcal { M }}_{v}$ on a set of 50 values in the interval $[0, \widehat{v})$. We have to compute numerically the needed integrals from (5.19) since we did not reach the symbolic values of them. For these values we have computed $\operatorname{det} \mathcal{M}_{v}$, too. We consider these values of $\operatorname{det} \mathcal{M}_{v}$ for a set of equidistant values in $[0, \widehat{v})$ and we use interpolation to find an approximation function $v \mapsto f(v)$ of the function $v \mapsto \operatorname{det} \mathcal{M}_{v}$, on $[0, \widehat{v})$.

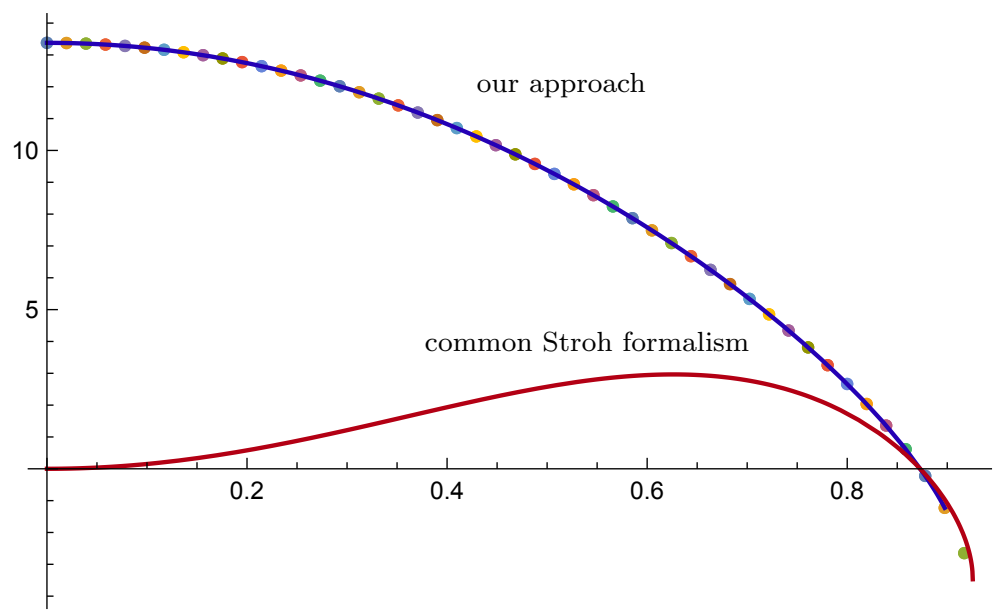

Figure 3: The plots of the approximation of $v \mapsto \operatorname{det} \mathcal{M}_{v}$ (blue curve) and of $v \mapsto s(v)$ (red curve), where $s(v)$ from (4.22) defines the secular equations in the Stroh formalism approach [11], with respect to the surface waves speed $v$ for the aluminum-epoxy composite. These two function have the same (unique) root in the interval $[0, \widehat{v}$ ).

After that, we find the root of $f$ on $[0, \widehat{v})$. Since this root given by mathematical software does not lead to a vanishing $\operatorname{det} \mathcal{M}_{v}$, we are looking in the neighbourhood of this root for a $v$ such that $\operatorname{det} \boldsymbol{M}_{v}$ is close to zero. Such a value is $\boldsymbol{v}_{\boldsymbol{R}}=\mathbf{0 . 8 7 3 0 3 5 2}$. This is the approximate value we have found for the wave speed, i.e. the approximate solution of our secular equation. We work with 7 decimals since the values obtained for the approximations of $\operatorname{det} \mathcal{M}_{v}$ are very sensitive to small changes of $v$.

We have considered the same coefficients in the secular equation established in [11] and we have remarked, see Figure 3, that both functions defining the secular equation in our form and the form given in [11], respectively, vanish in the same value in the interval $[0, \widehat{v})$. We have approximated also the solution of the secular equation $s(v)=0$ from [11] and we have found the approximation of the corresponding admissible wave speed to be 0.87296, which is not far from the approximate wave speed given by our secular equation $\operatorname{det} \boldsymbol{\mathcal { M }}_{v}=0$, i.e., $\boldsymbol{v}_{\boldsymbol{R}}=\mathbf{0 . 8 7 3 0 3 5 2}$. We mention we cannot obtain the precise value of $v_{R}$ and that a new numerical strategy or a better mathematical software could lead to a better accuracy.

Step 3: Construct the amplitudes and the solution. For $v=v_{R}$, we find $y(0)$ as solution of the algebraic system (5.5). Then we construct $y$ from (5.1). Finally, we construct the solution $\mathcal{U}$ from (3.1). We find the approximate solution of $(5.5)_{2}$ to be

$$
y(0)=\left(\begin{array}{c}
\varsigma \\
-1.66731 \mathrm{i} \varsigma \\
-0.0120298 \mathrm{i} \varsigma
\end{array}\right), \quad \varsigma \in \mathbb{C} .
$$

Since $\mathcal{M}_{v_{R}}$ is approximated by $\mathcal{M}_{v_{R}}=\left(\begin{array}{ccc}1.01413 & -0.608012 \mathrm{i} & 0.00513355 \mathrm{i} \\ 0.608012 \mathrm{i} & 0.365425 & -0.0463072 \\ -0.00513355 \mathrm{i} & -0.0463072 & 6.00576\end{array}\right)$, the matrix $\mathcal{E}_{v_{R}}=\mathcal{T}^{-1}\left(\mathcal{M}_{v_{R}}+\mathrm{i} \boldsymbol{R}^{T}\right)$ is numerically approximated by

$$
\mathcal{E}_{v_{R}}=\left(\begin{array}{ccc}
1.18322 & 0.282484 \mathrm{i} & 0.00598908 \mathrm{i} \\
0.785418 \mathrm{i} & 0.0712845 & -0.00904174 \\
0.00706743 \mathrm{i} & -0.00766037 & 0.993447
\end{array}\right)
$$


and, using (5.1), the function $y\left(x_{2}\right)$ is determined. Then, using $y\left(x_{2}\right):=\left(\begin{array}{ccc}\frac{1}{\sqrt{\rho}} & 0 & 0 \\ 0 & \frac{1}{\sqrt{\rho}} & 0 \\ 0 & 0 & \frac{1}{\sqrt{\rho j} \mu_{\mathrm{e}} \tau_{\mathrm{c}}^{2}}\end{array}\right)^{-1} z\left(x_{2}\right)$ we find $z\left(x_{2}\right)$. In the end, from (3.1) we find the solution

$$
\mathcal{U}\left(x_{1}, x_{2}, t\right)=\left(\begin{array}{l}
u_{1}\left(x_{1}, x_{2}, t\right) \\
u_{2}\left(x_{1}, x_{2}, t\right) \\
\vartheta_{3}\left(x_{1}, x_{2}, t\right)
\end{array}\right)=\operatorname{Re}\left[\left(\begin{array}{c}
z_{1}\left(x_{2}\right) \\
z_{2}\left(x_{2}\right) \\
\mathrm{i} z_{3}\left(x_{2}\right)
\end{array}\right) e^{\mathrm{i} k\left(x_{1}-v t\right)}\right]
$$

represented in Figure 4.

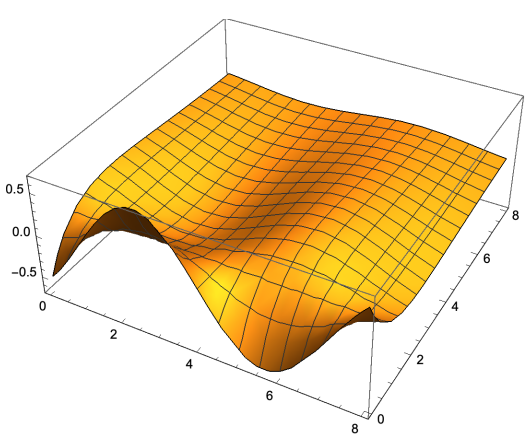

(a) Plot of the $u_{1}$-component of the displacement.

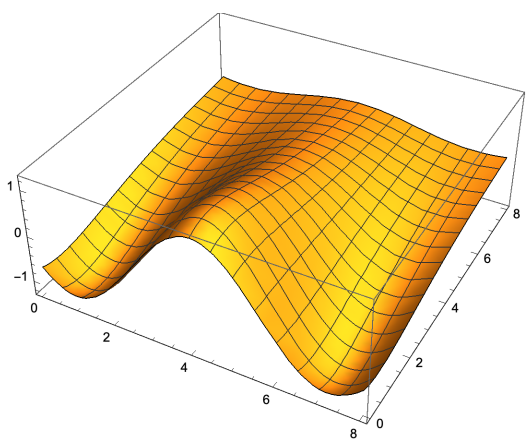

(b) Plot of the $u_{2}$-component of the displacement.

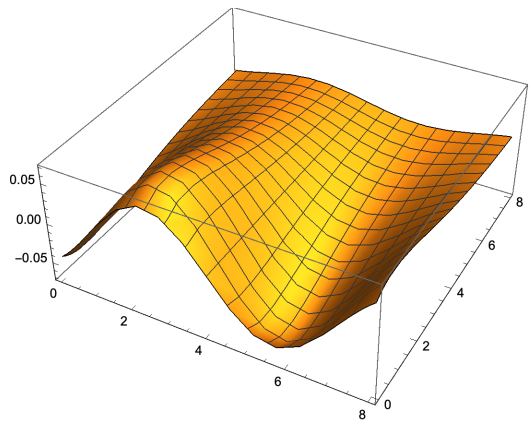

(c) Plot of $\vartheta_{3}$-component of the micro-rotation vector.

Figure 4: The plot of the solution at time $t=1$ and for the choice $\varsigma=\mathrm{i}$.

In Figure 5 we present the dependence of the wave speed on the wave number in the framework of the linear Cosserat theory and classical linear elasticity. In contrast to classical elasticity where the wave speed does not depend on the wave number, in the linear Cosserat model there is a dispersion curve describing such a dependency. Moreover, it seems that the speed goes asymptotically to a finite value of the wave speed for large values of the wave number. The computations for linear classical elasticity as limit case of the approach which is done in this paper for linear Cosserat elasticity are presented in more details in Section 7.

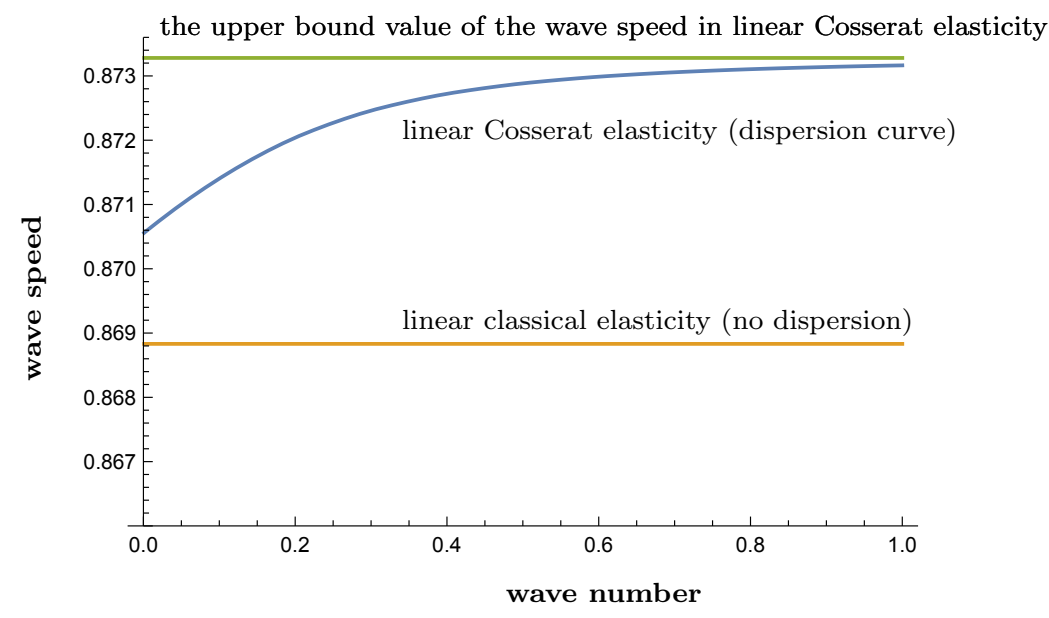

Figure 5: The dependence of the wave speed on the wave number for aluminum-epoxy composite $\left(\lambda_{\mathrm{e}}=7.59 \mathrm{GPa}, \mu_{\mathrm{c}}=\frac{\kappa}{2}=\right.$ $\left.0.0074466 \mathrm{GPa}, \mu_{\mathrm{e}}=\mu_{\text {Eringen }}+\frac{\kappa}{2}=1.89745 \mathrm{GPa}, \rho=2.22287 \frac{g}{\mathrm{~mm}^{3}}, j \mu_{\mathrm{e}} \tau_{\mathrm{c}}^{2}=0.0196 \mathrm{~mm}^{2}, \mu_{\mathrm{e}} L_{\mathrm{c}}^{2} \gamma=0.263383 \mathrm{GPa} \times \mathrm{mm}\right) \mathrm{vs}$. for a linear elastic material having the parameters $\left(\lambda=7.59 \mathrm{GPa}, \mu=1.89745 \mathrm{GPa}, \rho=2.22287 \frac{\mathrm{g}}{\mathrm{mm}^{3}}\right.$.) For the considered material parameters, the speed goes to 0.87327989 for large values of the wave speed.

From Figure 6 we observe that the frequency increases as function of the wave number since the wave speed increases as function of the wave number (see Figure 5) and $\omega(k)=k v(k)$. Moreover, since $0 \leq v(k)<\widehat{v}$, the 


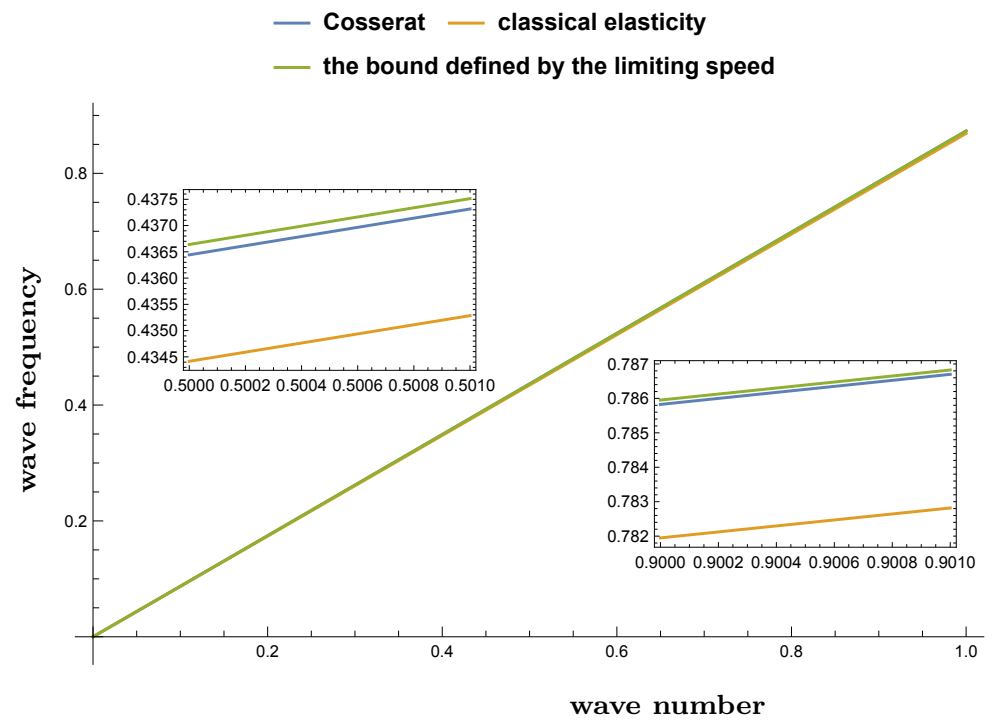

Figure 6: The dependence of the wave frequency on the wave number for aluminum-epoxy composite $\left(\lambda_{\mathrm{e}}=7.59 \mathrm{GPa}, \mu_{\mathrm{c}}=\frac{\kappa}{2}=\right.$ $\left.0.0074466 \mathrm{GPa}, \mu_{\mathrm{e}}=\mu_{\text {Eringen }}+\frac{\kappa}{2}=1.89745 \mathrm{GPa}, \rho=2.22287 \frac{g}{\mathrm{~mm}^{3}}, j \mu_{\mathrm{e}} \tau_{\mathrm{c}}^{2}=0.0196 \mathrm{~mm}^{2}, \mu_{\mathrm{e}} L_{\mathrm{c}}^{2} \gamma=0.263383 \mathrm{GPa} \times \mathrm{mm}\right) \mathrm{vs}$. for a linear elastic material having the parameters $\left(\lambda=7.59 \mathrm{GPa}, \mu=1.89745 \mathrm{GPa}, \rho=2.22287 \frac{\mathrm{g}}{\mathrm{mm}^{3}}\right.$. $)$ From the magnified windows and since the orange curve is linear (expressing the dependency on the wave frequency as function of the wave number in classical elasticity) we observe that the wave frequency as function of the wave number is not linear and we have dispersion. However, there are no band-gap. It is clear that dispersion curve lie under (on the right side of) all the dispersion curves of the real plane waves, since the $\omega(k)=k v(k)$ with $0 \leq v(k)<\widehat{v}(k)$ and the limiting speed $\widehat{v}(k)$ represent the minimum slope of all the possible dispersion curve at $k$, for the real plane waves.

mapping $k \rightarrow v(k)$ is bounded. In addition, the mapping $k \rightarrow v(k)$ is smooth. Therefore, the range of the mapping $k \rightarrow \omega(k)$ is $[0, \infty)$ and we conclude that for this material there is no band-gap. Even if, at a first look from Figure 6, it seems that the wave frequency depends linearly as function of the wave number (as in classical elasticity), from the magnified picture we see that this is not true. This is also clear since the wave speed is not constant as function of the wave number and therefore the group velocity $\frac{d \omega(k)}{d k}$ is not constant. Even if we have seen this aspect only for this material (we do not have an analytical proof yet), we extrapolate this remark and we conjecture that, in Cosserat elasticity the wave frequency is not linear as function of the wave number and we have dispersion. This can also be observed from Figure 7 in which the group velocity is given.

We will not explicit it here, but another numerical approach is also possible. We indicate it in the following:

II. The second algorithm:

Step 1: Identify the limiting speed $\widehat{v}$ : from (4.17) in our case, since this limiting speed was previously found by Chiriţă and Ghiba in [11] .

Step 2: Consider the hermitian matrix $\mathcal{M}_{v}$ in the form

$$
\mathcal{M}_{v}=\left(\begin{array}{ccc}
\mathcal{M}_{1} & \mathcal{M}_{3}+\mathrm{i} \mathcal{M}_{4} & \mathcal{M}_{5}+\mathrm{i} \mathcal{M}_{6} \\
\mathcal{M}_{3}-\mathrm{i} \mathcal{M}_{4} & \mathcal{M}_{2} & \mathcal{M}_{7}+\mathrm{i} \mathcal{M}_{8} \\
\mathcal{M}_{5}-\mathrm{i} \mathcal{M}_{6} & \mathcal{M}_{7}-\mathrm{i} \mathcal{M}_{8} & \mathcal{M}_{9}
\end{array}\right)
$$

and solve both the Riccati equation and the secular equation

$$
(\mathcal{M}-\mathrm{i} \mathcal{R}) \mathcal{T}^{-1}\left(\mathcal{M}+\mathrm{i} \mathcal{R}^{T}\right)-\mathcal{Q}+k^{2} v^{2} \mathbb{1}=0, \quad \operatorname{det} \mathcal{M}_{v}=0
$$

With the condition $(6.7)_{2}$ this matrix has two positive eigenvalues and one is a zero eigenvalue. Writing separately the real and imaginary part of the equation yields nine quadratic equation for the $\mathcal{M}_{i}(i=1,2 \ldots 9)$. Together with the secular equation we have an algebraic system of 10 equations for the 10 unknowns $\mathcal{M}_{1}, \mathcal{M}_{2}, \ldots, \mathcal{M}_{9}, v$.

i. Solve this nonlinear system. 


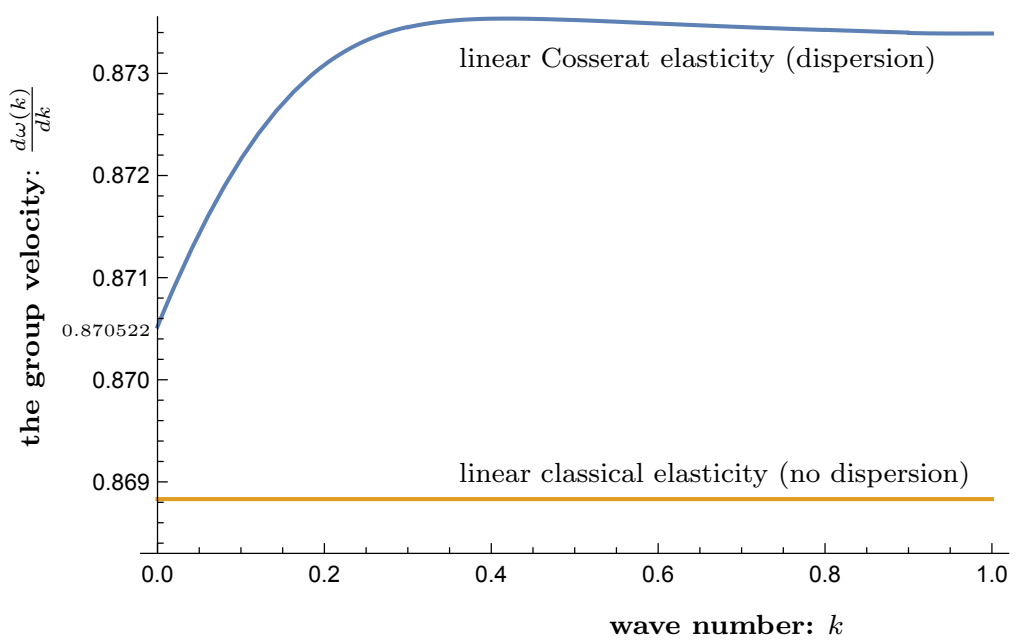

Figure 7: The dependence of the wave frequency on the wave number for aluminum-epoxy composite $\left(\lambda_{\mathrm{e}}=7.59 \mathrm{GPa}, \mu_{\mathrm{c}}=\frac{\kappa}{2}=\right.$ $\left.0.0074466 \mathrm{GPa}, \mu_{\mathrm{e}}=\mu_{\text {Eringen }}+\frac{\kappa}{2}=1.89745 \mathrm{GPa}, \rho=2.22287 \frac{g}{\mathrm{~mm}^{3}}, j \mu_{\mathrm{e}} \tau_{\mathrm{c}}^{2}=0.0196 \mathrm{~mm}^{2}, \mu_{\mathrm{e}} L_{\mathrm{c}}^{2} \gamma=0.263383 \mathrm{GPa} \times \mathrm{mm}\right) \mathrm{vs}$. for a linear elastic material having the parameters $\left(\lambda=7.59 \mathrm{GPa}, \mu=1.89745 \mathrm{GPa}, \rho=2.22287 \frac{g}{\mathrm{~mm}^{3}}.\right)$ From the magnified windows and since the orange curve is linear (expressing the dependency on the wave frequency as function of the wave number in classical elasticity) we observe that the wave frequency as function of the wave number is not linear and we have dispersion. However, there are no band-gap.

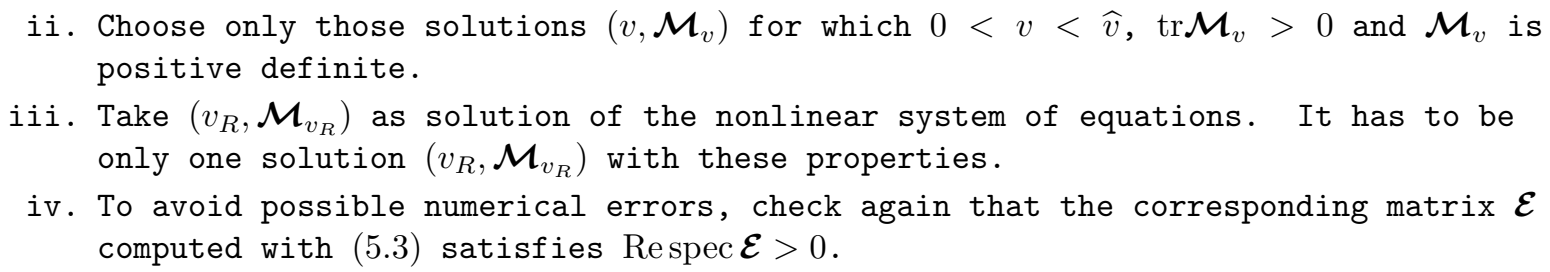

Step 3: For $v=v_{R}$ and $\mathcal{M}=\mathcal{M}_{v_{R}}$, find $y(0)$ as solution of the algebraic system (5.5) and construct $y\left(x_{2}\right)$ from $(5.1)$.

Step 4: Construct the solution $\mathcal{U}$ from $(3.1)$.

\section{From linear Cosserat theory to classical linear elasticity: A con- sistency check and comparison of the results}

In this section we rediscover the results from classical linear elasticity as a limit case of the results obtained in linear Cosserat theory. First, we remark that

$$
\| \mathrm{D} \text { axl } \mathbf{A} \|^{2}=0 \quad \text { implies that } \quad \text { axl } \mathbf{A}=\text { constant } \quad \text { in } \Omega,
$$

which corresponds to a time dependent rigid (macroscopic) movement of the entire body. In addition, under Dirichlet homogeneous boundary conditions on $\operatorname{axl} \mathbf{A}$ we find that axl $\mathbf{A}$ vanishes in the entire body at any time. is

In fact, by looking at the expression of the total energy, we observe that the energy due to the microrotation

$$
\begin{aligned}
& \rho j \mu_{\mathrm{e}} \tau_{\mathrm{c}}^{2}\|(\operatorname{axl} \mathbf{A}), t\|^{2}+\mu_{\mathrm{c}}\|\operatorname{skew}(\mathrm{D} u-\mathbf{A})\|^{2} \\
& +\frac{\mu_{\mathrm{e}} L_{\mathrm{c}}^{2}}{2}\left[a_{1}\|\operatorname{dev} \operatorname{sym}(\mathrm{D} \operatorname{axl} \mathbf{A})\|^{2}+a_{2}\|\operatorname{skew}(\mathrm{D} \operatorname{axl} \mathbf{A})\|^{2}+\frac{4 a_{3}}{3}[\operatorname{tr}(\mathrm{D} \operatorname{axl} \mathbf{A})]^{2}\right]
\end{aligned}
$$

and that the situation described by (7.1) is formally equivalent to the case

$$
L_{\mathrm{c}} \rightarrow \infty,
$$


since the total energy has to remain finite. Moreover, assuming also that

$$
\mu_{\mathrm{c}} \rightarrow 0 \quad \text { and } \quad \tau_{\mathrm{c}} \rightarrow 0, \text { too, }
$$

the entire energy due to the microrotation vanishes and we are back in the framework of classical linear elasticity, i.e., the elastic energy density is

$$
W(\mathrm{D} u)=\mu_{\mathrm{e}}\left\|\operatorname{dev}_{3} \operatorname{sym} \mathrm{D} u\right\|^{2}+\frac{2 \mu_{\mathrm{e}}+3 \lambda_{\mathrm{e}}}{6}[\operatorname{tr}(\mathrm{D} u)]^{2} .
$$

In the following we explain that all the results concerning the propagation of the seismic wave in the framework of Cosserat theory are still valid for Cosserat couple modulus $\mu_{\mathrm{c}} \rightarrow 0$, and we rediscover the results concerning the propagation of the seismic waves from classical linear elasticity without assuming further conditions on the internal length scale $L_{\mathrm{c}}$ or on the internal time scale $\tau_{\mathrm{c}}$ (as was the case for real plane waves), since the domain of the admissible speeds is already bounded and cannot reach large values defined by large values of the internal length scale $L_{\mathrm{c}}$ or small values of the internal time scale $\tau_{\mathrm{c}}$.

Letting $\mu_{\mathrm{c}} \rightarrow 0$, the matrices involved in the Riccati equation become

$$
\begin{aligned}
& \mathcal{T}:=k^{2}\left(\begin{array}{ccc}
\frac{\mu_{\mathrm{e}}}{\rho} & 0 & 0 \\
0 & \frac{2 \mu_{\mathrm{e}}+\lambda_{\mathrm{e}}}{\rho} & 0 \\
0 & 0 & \frac{\mu_{\mathrm{e}} L_{\mathrm{c}}^{2} \gamma}{\rho j \mu_{\mathrm{e}} \tau_{\mathrm{c}}^{2}}
\end{array}\right), \quad \mathcal{R}:=k\left(\begin{array}{ccc}
0 & k \frac{\lambda_{\mathrm{e}}}{\rho} & 0 \\
k \frac{\mu_{\mathrm{e}}}{\rho} & 0 & 0 \\
0 & 0 & 0
\end{array}\right), \\
& \mathcal{Q}:=\left(\begin{array}{ccc}
k^{2} \frac{2 \mu_{\mathrm{e}}+\lambda_{\mathrm{e}}}{\rho}-k^{2} v^{2} & 0 & 0 \\
0 & k^{2} \frac{\mu_{\mathrm{e}}}{\rho}-k^{2} v^{2} & 0 \\
0 & 0 & k^{2} \frac{\mu_{\mathrm{e}} L_{\mathrm{c}}^{2} \gamma}{\rho j \mu_{\mathrm{e}} \tau_{\mathrm{c}}^{2}}-k^{2} v^{2}
\end{array}\right) .
\end{aligned}
$$

Thus, we have

$$
\begin{aligned}
\mathcal{T}_{\theta} & =\left(\begin{array}{ccc}
\frac{k^{2} \mu_{\mathrm{e}} \cos ^{2}(\theta)}{\rho}+\frac{k^{2} \sin ^{2}(\theta)\left(\lambda_{\mathrm{e}}+2 \mu_{\mathrm{e}}-\rho v^{2}\right)}{\rho} & -\frac{1}{2} \sin (2 \theta)\left(\frac{k^{2} \lambda_{\mathrm{e}}}{\rho}+\frac{k^{2} \mu_{\mathrm{e}}}{\rho}\right) & 0 \\
-\frac{1}{2} \sin (2 \theta)\left(\frac{k^{2} \lambda_{\mathrm{e}}}{\rho}+\frac{k^{2} \mu_{\mathrm{e}}}{\rho}\right) & \frac{k^{2} \cos ^{2}(\theta)\left(\lambda_{\mathrm{e}}+2 \mu_{\mathrm{e}}\right)}{\rho}+\frac{k^{2} \sin ^{2}(\theta)\left(\mu_{\mathrm{e}}-\rho v^{2}\right)}{\rho} & 0 \\
0 & 0 & \frac{L_{\mathrm{c}}^{2} \gamma k^{2}}{\tau_{\mathrm{c}}^{2} j \rho}-\sin ^{2}(\theta) k^{2} v^{2}
\end{array}\right) \\
\mathcal{R}_{\theta} & \left(\begin{array}{ccc}
\frac{1}{2} \sin (2 \theta)\left(\frac{k^{2} \mu_{\mathrm{e}}}{\rho}-\frac{k^{2}\left(\lambda_{\mathrm{e}}+2 \mu_{\mathrm{e}}-\rho v^{2}\right)}{\rho}\right) & \frac{k^{2} \lambda_{\mathrm{e}} \cos ^{2}(\theta)}{\rho}-\frac{k^{2} \mu_{\mathrm{e}} \sin ^{2}(\theta)}{\rho} & 0 \\
\frac{k^{2} \mu_{\mathrm{e}} \cos ^{2}(\theta)}{\rho}-\frac{k^{2} \lambda_{\mathrm{e}} \sin ^{2}(\theta)}{\rho} & \frac{1}{2} \sin (2 \theta)\left(\frac{k^{2}\left(\lambda_{\mathrm{e}}+2 \mu_{\mathrm{e}}\right)}{\rho}-\frac{k^{2}\left(\mu_{\mathrm{e}}-\rho v^{2}\right)}{\rho}\right) & 0 \\
0 & 0 & \frac{1}{2} \sin (2 \theta) k^{2} v^{2}
\end{array}\right) \\
\mathcal{Q}_{\theta} & =\left(\begin{array}{ccc}
\frac{k^{2} \mu_{\mathrm{e}} \sin ^{2}(\theta)}{\rho}+\frac{k^{2} \cos ^{2}(\theta)\left(\lambda_{\mathrm{e}}+2 \mu_{\mathrm{e}}-\rho v^{2}\right)}{\rho} & \frac{1}{2} \sin (2 \theta)\left(\frac{k^{2} \lambda_{\mathrm{e}}}{\rho}+\frac{k^{2} \mu_{\mathrm{e}}}{\rho}\right) & 0 \\
\frac{1}{2} \sin (2 \theta)\left(\frac{k^{2} \lambda_{\mathrm{e}}}{\rho}+\frac{k^{2} \mu_{\mathrm{e}}}{\rho}\right) & \frac{k^{2} \sin ^{2}(\theta)\left(\lambda_{\mathrm{e}}+2 \mu_{\mathrm{e}}\right)}{\rho}+\frac{k^{2} \cos ^{2}(\theta)\left(\mu_{\mathrm{e}}-\rho v^{2}\right)}{\rho} & 0 \\
0 & 0 & \frac{L_{\mathrm{c}}^{2} \gamma k^{2}}{\tau_{\mathrm{c}}^{2} j \rho}-\cos ^{2}(\theta) k^{2} v^{2}
\end{array}\right) .
\end{aligned}
$$

Consequently, we deduce

$$
\begin{aligned}
& \mathcal{T}_{\theta}^{-1}=\left(\begin{array}{ccc}
\frac{\rho\left(\lambda_{\mathrm{e}} \cos ^{2}(\theta)+\frac{1}{2} \mu_{\mathrm{e}}(\cos (2 \theta)+3)-\rho v^{2} \sin ^{2}(\theta)\right)}{k^{2}\left(\rho v^{2} \sin ^{2}(\theta)-\mu_{\mathrm{e}}\right)\left(-\lambda_{\mathrm{e}}-2 \mu_{\mathrm{e}}+\rho v^{2} \sin ^{2}(\theta)\right)} & \frac{\rho \sin (\theta) \cos (\theta)\left(\lambda_{\mathrm{e}}+\mu_{\mathrm{e}}\right)}{k^{2}\left(\rho v^{2} \sin ^{2}(\theta)-\mu_{\mathrm{e}}\right)\left(-\lambda_{\mathrm{e}}-2 \mu_{\mathrm{e}}+\rho \sin ^{2}(\theta)\right)} & 0 \\
\frac{\rho \sin ^{2}(\theta) \cos (\theta)\left(\lambda_{\mathrm{e}}+\mu_{\mathrm{e}}\right)}{k^{2}\left(\rho v^{2} \sin ^{2}(\theta)-\mu_{\mathrm{e}}\right)\left(-\lambda_{\mathrm{e}}-2 \mu_{\mathrm{e}}+\rho v^{2} \sin ^{2}(\theta)\right)} & \frac{\rho\left(\lambda_{\mathrm{e}} \sin ^{2}(\theta)-\frac{1}{2} \mu_{\mathrm{e}}(\cos (2 \theta)-3)+\rho\left(-v^{2}\right) \sin ^{2}(\theta)\right)}{k^{2}\left(\rho v^{2} \sin ^{2}(\theta)-\mu_{\mathrm{e}}\right)\left(-\lambda_{\mathrm{e}}-2 \mu_{\mathrm{e}}+\rho v^{2} \sin ^{2}(\theta)\right)} & 0 \\
0 & 0 & \frac{\tau_{c}^{2} j \rho}{k^{2}\left(L_{\mathrm{c}}^{2} \gamma-\tau_{c}^{2} j \rho v^{2} \sin ^{2}(\theta)\right)}
\end{array}\right), \\
& \mathcal{T}_{\theta}^{-1} \mathcal{R}_{\theta}^{T}=\left(\begin{array}{ccc}
\sin (2 \theta)\left(\frac{\mu_{\mathrm{e}}}{\lambda_{\mathrm{e}}+2 \mu_{\mathrm{e}}-\rho v^{2} \sin ^{2}(\theta)}+\frac{\rho v^{2}-2 \mu_{\mathrm{e}}}{2 \mu_{\mathrm{e}}-2 \rho v^{2} \sin ^{2}(\theta)}\right) & \frac{2 \mu_{\mathrm{e}}^{2} \cos ^{2}(\theta)+\lambda_{\mathrm{e}}\left(\mu_{\mathrm{e}} \cos (2 \theta)+\rho v^{2} \sin ^{2}(\theta)\right)}{\left(\rho v^{2} \sin ^{2}(\theta)-\mu_{\mathrm{e}}\left(-\lambda_{\mathrm{e}}-2 \mu_{\mathrm{e}}+\rho v^{2} \sin ^{2}(\theta)\right)\right.} & 0 \\
\frac{\mu_{\mathrm{e}}\left(\lambda_{\mathrm{e}} \cos (2 \theta)+\sin ^{2}(\theta)\left(\rho v^{2}-2 \mu_{\mathrm{e}}\right)\right)}{\left(\rho v^{2} \sin ^{2}(\theta)-\mu_{\mathrm{e}}\right)\left(-\lambda_{\mathrm{e}}-2 \mu_{\mathrm{e}}+\rho v^{2} \sin ^{2}(\theta)\right)} & \frac{\sin (2 \theta)\left(\mu_{\mathrm{e}}\left(2 \lambda_{\mathrm{e}}+2 \mu_{\mathrm{e}}+\rho v^{2}\right)-\rho^{2} v^{4} \sin ^{2}(\theta)\right)}{2\left(\rho v^{2} \sin ^{2}(\theta)-\mu_{\mathrm{e}}\right)\left(-\lambda_{\mathrm{e}}-2 \mu_{\mathrm{e}}+\rho v^{2} \sin ^{2}(\theta)\right)} & 0 \\
0 & 0 & \frac{1}{\frac{L_{c}^{2} \gamma \csc (\theta) \sec (\theta)}{\tau_{\mathrm{c}}^{2} j \rho v^{2}}-\tan (\theta)}
\end{array}\right) .
\end{aligned}
$$


Hence, the matrices $\mathbf{H}_{v}$ and $\mathbf{S}_{v}$ from (5.19) are

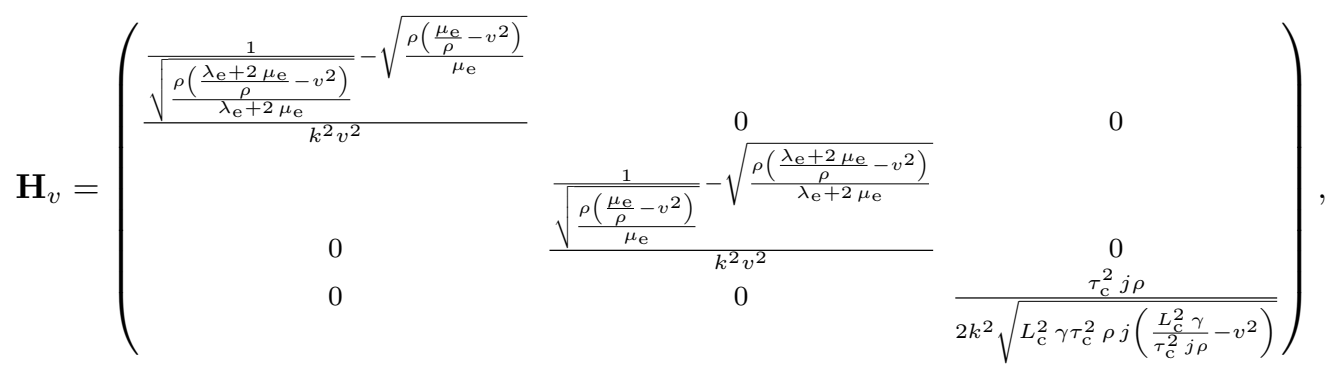

$$
\begin{aligned}
& =\left(\begin{array}{ccc}
\frac{c_{l}}{\sqrt{c_{l}^{2}-v^{2}}-\frac{\sqrt{c_{t}^{2}-v^{2}}}{c_{t}}} & 0 & 0 \\
k^{2} v^{2} & \frac{c_{t}}{\sqrt{c_{t}^{2}-v^{2}}-\frac{\sqrt{c_{l}^{2}-v^{2}}}{c_{l}}} & 0 \\
0 & 0 & \frac{c_{m}}{2 k^{2} \sqrt{c_{m}^{2}-v^{2}}}
\end{array}\right) \\
& \mathbf{S}_{v}=\left(\begin{array}{ccc}
0 & \frac{2 \sqrt{\mu_{\mathrm{e}}\left(\frac{\mu_{\mathrm{e}}}{\rho}-v^{2}\right)\left(\frac{\lambda_{\mathrm{e}}+2 \mu_{\mathrm{e}}}{\rho}-v^{2}\right)}-\sqrt{\lambda_{\mathrm{e}}+2 \mu_{\mathrm{e}}}\left(\frac{2 \mu_{\mathrm{e}}}{\rho}-v^{2}\right)}{\sqrt{\rho} v^{2} \sqrt{\frac{\lambda_{\mathrm{e}}+2 \mu_{\mathrm{e}}}{\rho}-v^{2}}} & 0 \\
-\frac{2 \sqrt{\mu_{\mathrm{e}}\left(\frac{\mu_{\mathrm{e}}}{\rho}-v^{2}\right)\left(\frac{\lambda_{\mathrm{e}}+2 \mu_{\mathrm{e}}}{\rho}-v^{2}\right)}-\sqrt{\lambda_{\mathrm{e}}+2 \mu_{\mathrm{e}}}\left(\frac{2 \mu_{\mathrm{e}}}{\rho}-v^{2}\right)}{v^{2} \sqrt{\lambda_{\mathrm{e}}+2 \mu_{\mathrm{e}}} \sqrt{\frac{\rho}{\mu_{\mathrm{e}}}} \sqrt{\frac{\mu_{\mathrm{e}}}{\rho}-v^{2}}} & 0 & 0 \\
0 & 0 & 0
\end{array}\right) \\
& =\left(\begin{array}{ccc}
0 & \frac{2 c_{t} \sqrt{\left(c_{t}^{2}-v^{2}\right)\left(c_{l}^{2}-v^{2}\right)}-c_{l}\left(2 c_{t}^{2}-v^{2}\right)}{v^{2} \sqrt{c_{l}^{2}-v^{2}}} & 0 \\
-\frac{2 c_{t} \sqrt{\left(c_{t}^{2}-v^{2}\right)\left(c_{l}^{2}-v^{2}\right)}-c_{l}\left(2 c_{t}^{2}-v^{2}\right)}{\frac{c_{l} v^{2} \sqrt{c_{t}^{2}-v^{2}}}{c_{t}}} & 0 & 0 \\
0 & 0 & 0
\end{array}\right) \text {, }
\end{aligned}
$$

where

$$
c_{l}=\sqrt{\frac{2 \mu_{\mathrm{e}}+\lambda_{\mathrm{e}}}{\rho}}, \quad c_{t}=\sqrt{\frac{\mu_{\mathrm{e}}}{\rho}}, \quad c_{m}=\sqrt{\frac{L_{\mathrm{c}}^{2} \gamma}{\rho j \tau_{\mathrm{c}}^{2}}}
$$

Note that for $\mu_{\mathrm{c}} \rightarrow 0$ we have

$$
\mathfrak{c}_{p}=c_{l}, \quad \mathfrak{c}_{s}=c_{t}, \quad \mathfrak{c}_{m_{1}}=c_{m}, \quad \mathfrak{c}_{m_{2}}=\min \left\{\mathfrak{c}_{s}, \mathfrak{c}_{m_{1}}\right\}=\min \left\{c_{t}, c_{m}\right\},
$$

and that a similar proof with that of Proposition 4.3 holds true in the case $\mu_{\mathrm{c}} \rightarrow 0$, too.

Proposition 7.1. For $2 \mu_{\mathrm{e}}+\lambda_{\mathrm{e}}>0, \mu_{\mathrm{e}}>0, \gamma>0$, the limiting speed in Cosserat elastic materials is given by

$$
\widehat{v}:=\inf _{\theta \in\left(-\frac{\pi}{2}, \frac{\pi}{2}\right)} v_{\theta} \equiv \min \left\{c_{l}, c_{t}, c_{m}\right\} .
$$

By considering $\mu_{\mathrm{c}} \rightarrow 0$, Theorem 5.4 allows us to affirm that if the constitutive coefficients satisfy the conditions $2 \mu_{\mathrm{e}}+\lambda_{\mathrm{e}}>0, \mu_{\mathrm{e}}>0, \gamma>0$, and $0 \leq v<\widehat{v}=\inf _{\theta \in\left(-\frac{\pi}{2}, \frac{\pi}{2}\right)} v_{\theta} \equiv \min \left\{c_{l}, c_{t}, c_{4}\right\}$, then the unique solution of the algebraic Riccati equation (5.5) that satisfies $\operatorname{Re}\left[\operatorname{spec}\left(\mathcal{T}^{-1}\left(\mathcal{M}+\mathrm{i} R^{T}\right)\right)\right]>0$ given by (5.19) takes the form

$$
\mathcal{M}_{v}=\left(\begin{array}{ccc}
\frac{c_{t} k^{2} v^{2} \sqrt{c_{l}^{2}-v^{2}}}{c_{l} c_{t}-\sqrt{c_{l}^{2}-v^{2}} \sqrt{c_{t}^{2}-v^{2}}} & \frac{\mathrm{i} c_{t} k^{2}\left(2 c_{t} \sqrt{\left(v^{2}-c_{l}^{2}\right)\left(v^{2}-c_{t}^{2}\right)}+c_{l}\left(v^{2}-2 c_{t}^{2}\right)\right)}{c_{l} c_{t}-\sqrt{c_{l}^{2}-v^{2}} \sqrt{c_{t}^{2}-v^{2}}} & 0 \\
\frac{\mathrm{i} c_{t} k^{2}\left(2 c_{t} \sqrt{\left(v^{2}-c_{l}^{2}\right)\left(v^{2}-c_{t}^{2}\right)}+c_{l}\left(v^{2}-2 c_{t}^{2}\right)\right)}{\sqrt{c_{l}^{2}-v^{2}} \sqrt{c_{t}^{2}-v^{2}}-c_{l} c_{t}} & \frac{c_{l} k^{2} v^{2} \sqrt{c_{t}^{2}-v^{2}}}{c_{l} c_{t}-\sqrt{c_{l}^{2}-v^{2}} \sqrt{c_{t}^{2}-v^{2}}} & 0 \\
0 & 0 & \frac{2 k^{2} \sqrt{c_{m}^{2}-v^{2}}}{c_{m}}
\end{array}\right) .
$$


The secular equation in the Mielke-Fu's form is

$$
0=\operatorname{det} \mathcal{M}_{v}=\frac{2 k^{2} \sqrt{c_{m}^{2}-v^{2}}}{c_{m}} \frac{c_{l} c_{t} v^{4} \sqrt{c_{l}^{2}-v^{2}} \sqrt{c_{t}^{2}-v^{2}}-c_{t}^{2}\left(2 c_{t} \sqrt{\left(c_{l}^{2}-v^{2}\right)\left(c_{t}^{2}-v^{2}\right)}+c_{l}\left(v^{2}-2 c_{t}^{2}\right)\right)^{2}}{\left(c_{l} c_{t}-\sqrt{c_{l}^{2}-v^{2}} \sqrt{c_{t}^{2}-v^{2}}\right)^{2}} .
$$

An admissible wave speed has to satisfy $0 \leq v<\widehat{v}=\inf _{\theta \in\left(-\frac{\pi}{2}, \frac{\pi}{2}\right)} v_{\theta} \equiv \min \left\{c_{l}, c_{t}, c_{m}\right\}$. Therefore, the secular equation in the Mielke-Fu's form is equivalent to

$$
0=s_{\text {Mielke }-\mathrm{Fu}}(v) \equiv \frac{c_{l} c_{t} v^{4} \sqrt{c_{l}^{2}-v^{2}} \sqrt{c_{t}^{2}-v^{2}}-c_{t}^{2}\left(2 c_{t} \sqrt{\left(c_{l}^{2}-v^{2}\right)\left(c_{t}^{2}-v^{2}\right)}+c_{l}\left(v^{2}-2 c_{t}^{2}\right)\right)^{2}}{\left(c_{l} c_{t}-\sqrt{c_{l}^{2}-v^{2}} \sqrt{c_{t}^{2}-v^{2}}\right)^{2}} .
$$

We mention that Mielke and Fu did not write explicitly this equation for elastic isotropic material. Since

$$
\lim _{v \rightarrow 0} \frac{c_{l} c_{t} v^{4} \sqrt{c_{l}^{2}-v^{2}} \sqrt{c_{t}^{2}-v^{2}}-c_{t}^{2}\left(2 c_{t} \sqrt{\left(c_{l}^{2}-v^{2}\right)\left(c_{t}^{2}-v^{2}\right)}+c_{l}\left(v^{2}-2 c_{t}^{2}\right)\right)^{2}}{\left(c_{l} c_{t}-\sqrt{c_{l}^{2}-v^{2}} \sqrt{c_{t}^{2}-v^{2}}\right)^{2}}=-4 c_{t}^{4} k^{4}
$$

the value $v=0$ is not a singular point of $\operatorname{det} \mathcal{M}_{v}$ and the secular equation reduces to

$$
c_{l} c_{t} v^{4} \sqrt{c_{l}^{2}-v^{2}} \sqrt{c_{t}^{2}-v^{2}}-c_{t}^{2}\left(2 c_{t} \sqrt{\left(c_{l}^{2}-v^{2}\right)\left(c_{t}^{2}-v^{2}\right)}+c_{l}\left(v^{2}-2 c_{t}^{2}\right)\right)^{2}=0 .
$$

The secular equation (7.15) and its equivalent form (7.17) do not coincide with the classical well known form of the secular equation, i.e.

$$
0=s_{\text {classic }} \equiv \sqrt{\left(1-\frac{v^{2}}{c_{l}^{2}}\right)\left(1-\frac{v^{2}}{c_{t}^{2}}\right)}-\left(2-\frac{v^{2}}{c_{t}^{2}}\right)^{2} .
$$

Indeed, even when the Stroh formalism is used $[17,16]$, the obtained secular equation does not coincide with the classical form, and it is given by

$$
s_{\text {Stroh }} \equiv \frac{\rho v^{2} \sqrt{c_{11}-\rho v^{2}} \sqrt{c_{66}-\rho v^{2}}}{\sqrt{c_{11} c_{66}}}-\frac{\left(\rho v^{2}-c_{0}\right)\left(\rho v^{2}-c_{66}\right)}{c_{66}}
$$

where

$$
c_{12}=\lambda_{\mathrm{e}}, \quad c_{66}=\mu_{\mathrm{e}}, \quad c_{11}=\lambda_{\mathrm{e}}+2 \mu_{\mathrm{e}}, \quad c_{0}=c_{11}-\frac{c_{12}^{2}}{c_{11}} .
$$

However, all these three forms of the secular equations are equivalent in the sense that they predict the same wave speed in the same domain of admissible wave speeds. We illustrate this numerically, see Figure 8 , for a specific material which is related to aluminium-epoxy. We point out again that there is no dependence of the wave speed on the wave number $k$, contrary with what happens when the Cosserat theory is considered, see Figure 5 .

Moreover, after solving the system $\mathcal{M}_{v_{\mathrm{R}}} y(0)=0$ we obtain that the last component of $y(0)$ vanishes, since $\frac{2 k^{2} \sqrt{c_{m}^{2}-v_{\mathrm{R}}^{2}}}{c_{m}} \neq 0$ due to the fact the solution is in the admissible set, which implies that $v_{\mathrm{R}}<c_{m}$. This means that the micro-rotation vanishes and only macroscopic displacements govern the movement of the half-space. Therefore, from (5.1), the wave propagation solution will be

$$
y\left(x_{2}\right)=e^{-k x_{2} \mathcal{E}_{v_{\mathrm{R}}}}\left(\begin{array}{l}
y_{1}(0) \\
y_{2}(0)
\end{array}\right)
$$

where $\mathcal{E}_{v_{\mathrm{R}}} \in \mathbb{C}^{3 \times 3}$ is given by

$$
\mathcal{E}_{v_{\mathrm{R}}}=\mathcal{T}^{-1}\left(\mathcal{M}_{v_{\mathrm{R}}}+\mathrm{i} \mathcal{R}^{T}\right)
$$

and $y_{1}(0), y_{2}(0)$ are solutions of the algebraic system

$$
\left(\begin{array}{cc}
c_{t} k^{2} v^{2} \sqrt{c_{l}^{2}-v^{2}} \\
-\mathrm{i} c_{t} k^{2}\left(2 c_{t} \sqrt{\left(v^{2}-c_{l}^{2}\right)\left(v^{2}-c_{t}^{2}\right)}+c_{l}\left(v^{2}-2 c_{t}^{2}\right)\right) & \mathrm{i} c_{t} k^{2}\left(2 c_{t} \sqrt{\left(v^{2}-c_{l}^{2}\right)\left(v^{2}-c_{t}^{2}\right)}+c_{l}\left(v^{2}-2 c_{t}^{2}\right)\right) \\
c_{l} k^{2} v^{2} \sqrt{c_{t}^{2}-v^{2}}
\end{array}\right)\left(\begin{array}{l}
y_{1}(0) \\
y_{2}(0)
\end{array}\right)=0 .
$$




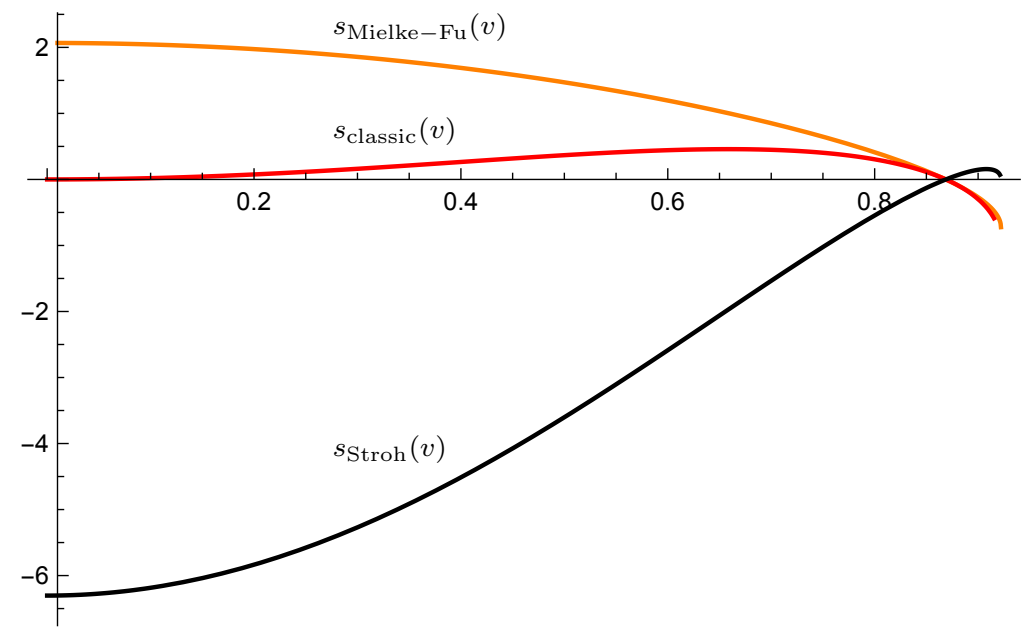

Figure 8: All the alternative forms of the secular equations are equivalent. For a linear elastic material having the parameters $\lambda_{\mathrm{e}}=7.59 \mathrm{GPa}, \mu_{\mathrm{e}}=1.89745 \mathrm{GPa}, \rho=2.22287 \frac{\mathrm{g}}{\mathrm{mm}^{3}}$ they give us the same value of the wave speed, i.e., $v_{\mathrm{R}}=0.868832$.

\section{Final remarks}

In this paper we have shown that the approach proposed by Mielke and Fu [24, 47] for the study of the propagation of seismic waves in anisotropic linear elastic materials can be used in the framework of the isotropic Cosserat elastic materials, too. One of the big advantages of this new approach, compared with the classical methods like Stroh formalism, is that it leads to the proof of the existence and uniqueness of the solution of the obtained secular equation. While the existence of solutions was proven before for several problems concerning the propagation of seismic waves in materials with microstructure [10,11], but using restrictive conditions upon the constitutive coefficients, the uniqueness of the solution was, in the best situation, only conjectured in such generalized theories. Indeed, the problem of existence and uniqueness of the solution of the secular equation remains unsolved in almost all generalized linear theories from elasticity. This is the case since the explicit forms of the corresponding secular equations are not completely analytically written. It is not the case when Mielke and Fu's approach is used [24, 47], the secular equation being written as $\operatorname{det} \boldsymbol{\mathcal { M }}_{v}=0$, where the hermitian matrix $\mathcal{M}_{v}$ has a known integral form. Moreover, due to the form of the hermitian matrix, it is possible to prove that the secular equation $\operatorname{det} \boldsymbol{\mathcal { M }}_{v}=0$ has a unique admissible solution. Therefore, with this paper we close the problem of the propagation of seismic waves in isotropic linear Cosserat elastic materials and we propose two numerically viable strategies to solve this problem for specific materials.

\section{Acknowledgement.}

The work of Ionel-Dumitrel Ghiba was supported by a grant of the Romanian Ministry of Research and Innovation, CNCSUEFISCDI, project number PN-III-P1-1.1-TE- 2021-0783, within PNCDI III.

Hassam Khan thanks HEC/DAAD overseas scholarship scheme for MSc leading to PhD, 2016 (57343333).

Angela Madeo acknowledges support from the European Commission through the funding of the ERC Consolidator Grant META-LEGO, N 101001759.

Patrizio Neff acknowledges support in the framework of the Priority Programme SPP 2256 "Variational Methods for Predicting Complex Phenomena in Engineering Structures and Materials" funded by the Deutsche Forschungsgemeinschaft (DFG, German research foundation), (Project-ID 440935806) "A variational scale-dependent transition scheme - from Cauchy elasticity to the relaxed micromorphic continuum".

\section{References}

[1] J.D. Achenbach. Wave Propagation in Elastic Solids. North-Holland Publishing Company, Amsterdam, 1973.

[2] H. Altenbach, V. Eremeyev, L. Lebedev, and L.A. Rendón. Acceleration waves and ellipticity in thermoelastic micropolar media. Arch. Appl. Mech., 80(3):217-227, 2010.

[3] S. Barnet. Matrix Methods and Applications, 1990.

[4] D.M. Barnett and J. Lothe. Consideration of the existence of surface wave (Rayleigh wave) solutions in anisotropic elastic crystals. J. Phys. F: Metal Physics, 4(5):671, 1974. 
[5] D.M. Barnett and J. Lothe. Free surface (Rayleigh) waves in anisotropic elastic half-spaces: the surface impedance method. Proc. Roy. Soc. London. A, 402(1822):135-152, 1985.

[6] D.M. Barnett, J. Lothe, K. Nishioka, and R.J. Asaro. Elastic surface waves in anisotropic crystals: a simplified method for calculating Rayleigh velocities using dislocation theory. J. Physics F: Metal Physics, 3(6):1083, 1973.

[7] S.V. Biryukov. Impedance method in the theory of elastic surface-waves. Soviet Physics Acoustics, 31(5):350-354, 1985.

[8] Ph. Boulanger and M. Hayes. Bivectors and Waves in Mechanics and Optics, volume 4. CRC Press, 1993.

[9] P. Chadwick and G.D. Smith. Foundations of the theory of surface waves in anisotropic elastic materials. In Adv. Appl. Mech., volume 17, pages 303-376. 1977.

[10] S. Chiriţă and I. D. Ghiba. Inhomogeneous plane waves in elastic materials with voids. Wave Motion, 47:333-342, 2010.

[11] S. Chiriţă and I. D. Ghiba. Rayleigh waves in Cosserat elastic materials. Int. J. Eng. Sci., 51:117-127, 2012.

[12] W.D. Claus and A.C. Eringen. Three dislocation concepts and micromorphic mechanics. In Developments in Mechanics, Proceedings of the 12th Midwestern Mechanics Conference, volume 6, pages 349-358. Midwestern, 1969.

[13] W.D. Claus and A.C. Eringen. Dislocation dispersion of elastic waves. Int. J. Engng. Sci., 9:605-610, 1971.

[14] P.K. Currie. Rayleigh waves on elastic crystals. Q. J. Mech. Appl. Math., 27(4):489-496, 1974.

[15] M. Destrade. The explicit secular equation for surface acoustic waves in monoclinic elastic crystals. J. Acoust. Soc. Am, 109(4):1398-1402, 2001.

[16] M. Destrade. Seismic Rayleigh waves on an exponentially graded, orthotropic half-space. Proc. R. Soc. London A, 463(2078):495-502, 2007.

[17] M. Destrade. Seismic rayleigh waves on an exponentially graded, orthotropic half-space. Proc. R. Soc. A, 463:495-502, 2007.

[18] M. Destrade, P. Martin, and T.C.T. Ting. The incompressible limit in linear anisotropic elasticity, with applications to surface waves and elastostatics. J. Mech. Phys. Sol., 50(7):1453-1468, 2002.

[19] V.A. Eremeyev. Acceleration waves in micropolar elastic media. Doklady Physics, 50:204-206, 2005.

[20] A.C. Eringen. Microcontinuum Field Theories. Springer, Heidelberg, 1999.

[21] A.C. Eringen and W.D. Claus. A micromorphic approach to dislocation theory and its relation to several existing theories. In J.A. Simmons, R. de Wit, and R. Bullough, editors, Fundamental Aspects of Dislocation Theory., volume 1 of Nat. Bur. Stand. (U.S.), Spec. Publ., pages 1023-1040. Spec. Publ., 1970.

[22] A.C. Eringen and E.S. Suhubi. Nonlinear theory of simple micro-elastic solids. I. Int. J. Eng. Sci., 2:189-203, 1964.

[23] V. I. Erofeyev. Wave Processes in Solids with Microstructure. World Scientific, New Jersey, 2003.

[24] Y.B. Fu and A. Mielke. A new identity for the surface-impedance matrix and its application to the determination of surfacewave speeds. Proc. Roy. Soc. London. A: Math., Phys. Eng. Sci., 458(2026):2523-2543, 2002.

[25] Y.B. Fu and R.W. Ogden. Nonlinear Elasticity: Theory and Applications, volume 281. Cambridge University Press, 2001.

[26] R.D. Gauthier. Experimental investigations on micropolar media. In O. Brulin and R.K.T. Hsieh, editors, Mechanics of Micropolar Media, pages 395-463. CISM Lectures, World Scientific, Singapore, 1982.

[27] E.F. Grekova, M.A. Kulesh, and G.C. Herman. Waves in linear elastic media with microrotations, Part 2: Isotropic reduced Cosserat model. Bull. Seismol. Soc. Am., 99(2B):1423-1428, 2009.

[28] S. Hassanpour and G.R. Heppler. Micropolar elasticity theory: a survey of linear isotropic equations, representative notations, and experimental investigations. Math. Mech. Solids, 22(2):224-242, 2017.

[29] M. Hayes. Inhomogeneous plane waves. In The Breadth and Depth of Continuum Mechanics, pages 247-285. Springer, 1986.

[30] M. Hayes and R.S. Rivlin. A note on the secular equation for Rayleigh waves. Z. Angew. Math. Phys., 13(1):80-83, 1962.

[31] K.A. Ingebrigtsen and A. Tonning. Elastic surface waves in crystals. Phys. Rev., 184(3):942, 1969.

[32] J. Jeong and P. Neff. Existence, uniqueness and stability in linear Cosserat elasticity for weakest curvature conditions. Math. Mech. Solids, 15(1):78-95, 2010.

[33] H.W. Knobloch, A. Isidori, and D. Flockerzi. Topics in Control Theory, volume 22. Birkhäuser, 2012.

[34] R.H. Koebke and Y. Weitsman. Surface wave propagation over an elastic Cosserat half-space. J. Acoust. Soc. Am., 50:875-884, 1971.

[35] M.A. Kulesh, E.F. Grekova, and I.N. Shardakov. The problem of surface wave propagation in a reduced Cosserat medium. Acoust. Phys., 55(2):218-226, 2009.

[36] M.A. Kulesh, V.P. Matveenko, and I.N. Shardakov. Exact analytical solution of the Kirsch problem within the framework of the Cosserat continuum and pseudocontinuum. J. Appl. Mech. Tech. Phys., 42:687-695, 2001.

[37] M.A. Kulesh, V.P. Matveenko, and I.N. Shardakov. Parametric analysis of analytical solutions to one- and two-dimensional problems in couple-stress theory of elasticity. Z. Angew. Math. Mech., 83:238-248, 2003.

[38] M.A. Kulesh, V.P. Matveenko, and I.N. Shardakov. Construction and analysis of an analytical solution for the surface Rayleigh wave within the framework of the Cosserat continuum. J. Appl. Mech. Tech. Phys., 46:556-563, 2005.

[39] M.A. Kulesh, V.P. Matveenko, and I.N. Shardakov. Propagation of surface elastic waves in the Cosserat medium. Acoust. Phys., 52: 186-193, 2006. 
[40] M. Lazar and H. Kirchner. Cosserat (micropolar) elasticity in Stroh form. Int. J. Solids Struct., 42(20):5377-5398, 2005.

[41] X.-F. Li. On approximate analytic expressions for the velocity of Rayleigh waves. Wave Motion, 44:120-127, 2006.

[42] J. Lothe and D.M. Barnett. On the existence of surface-wave solutions for anisotropic elastic half-spaces with free surface. $J$. Appl. Phys., 47(2):428-433, 1976.

[43] A. Madeo, P. Neff, I. D. Ghiba, L. Placidi, and G. Rosi. Band gaps in the relaxed linear micromorphic continuum. Z. Angew. Math. Mech., doi 10.1002 / zamm.201400036, 2014.

[44] A. Madeo, P. Neff, I. D. Ghiba, L. Placidi, and G. Rosi. Wave propagation in relaxed linear micromorphic continua: modelling metamaterials with frequency band-gaps. Cont. Mech. Therm., 27:551-570, 2015.

[45] A. Madeo, P. Neff, I.D. Ghiba, and G. Rosi. Reflection and transmission of elastic waves in non-local band-gap metamaterials: a comprehensive study via the relaxed micromorphic model. J. Mech. Phys. Solids, 95:441-479, 2016.

[46] P.G. Malischewsky. Comment to "A new formula for velocity of Rayleigh waves" by D. Nkemzi [Wave Motion 26 (1997) 199-205]. Wave Motion, 31:93-96, 2000.

[47] A. Mielke and Y.B. Fu. Uniqueness of the surface-wave speed: a proof that is independent of the Stroh formalism. Math. Mech. Solids, 9(1):5-15, 2004.

[48] A. Mielke and P. Sprenger. Quasiconvexity at the boundary and a simple variational formulation of Agmon's condition. J. Elasticity, 51(1):23-41, 1998.

[49] R.D. Mindlin. Micro-structure in linear elasticity. Arch. Rat. Mech. Anal., 16:51-77, 1964.

[50] V.G. Mozhaev. Some new ideas in the theory of surface acoustic waves in anisotropic media. In IUTAM Symposium on Anisotropy, Inhomogeneity and Nonlinearity in Solid Mechanics, pages 455-462, 1995.

[51] P. Neff, I. D. Ghiba, M. Lazar, and A. Madeo. The relaxed linear micromorphic continuum: well-posedness of the static problem and relations to the gauge theory of dislocations. Q. J. Mech. Appl. Math., 68:53-84, 2015.

[52] P. Neff, I. D. Ghiba, A. Madeo, L. Placidi, and G. Rosi. A unifying perspective: the relaxed linear micromorphic continuum. Cont. Mech. Therm., 26:639-681, 2014.

[53] P. Neff, A. Madeo, G. Barbagallo, M.V. d'Agostino, R. Abreu, and I.D. Ghiba. Real wave propagation in the isotropic-relaxed micromorphic model. Proc. R. Soc. A, 473(2197):20160790, 2017.

[54] P. Neff and I. Münch. Curl bounds Grad on SO(3). ESAIM: Control, Optimisation and Calculus of Variations, 14(1):148-159, 2008.

[55] D. Nkemzi. A new formula for the velocity of Rayleigh waves. Wave Motion, 26:199-205, 1997.

[56] D. Nkemzi. A simple and explicit algebraic expression for the Rayleigh wave velocity. Mech. Res. Commun., 35:201-205, 2008.

[57] J.W. Nunziato and S.C. Cowin. A nonlinear theory of elastic materials with voids. Arch. Rat. Mech. Anal., 72:175-201, 1979.

[58] J.F. Nye. Some geometrical relations in dislocated crystals. Acta Metall., 1:153-162, 1953.

[59] M. Rahman and J.R. Barber. Exact expression for the roots of the secular equation for Rayleigh waves. ASME J. Appl. Mech., 62:250-252, 1995.

[60] Lord Rayleigh. On waves propagated along the plane surface of an elastic solid. Proc. London Math. Soc., 17:4-11, 1885.

[61] M. Shirani, D.J. Steigmann, and P. Neff. The Legendre-Hadamard condition in Cosserat elasticity theory. Q. J. Mech. Appl. Math., 73:293-303, 2020.

[62] B. Straughan. Stability and Wave Motion in Porous Media, volume 165. Springer Science \& Business Media, 2008.

[63] A.N. Stroh. Dislocations and cracks in anisotropic elasticity. Philos. Mag., 3(30):625-646, 1958.

[64] A.N. Stroh. Steady state problems in anisotropic elasticity. J. Math. Phys., 41(1-4):77-103, 1962.

[65] J.L. Synge. Elastic waves in anisotropic media. J. Math. Phys., 35(1-4):323-334, 1956.

[66] R.M. Taziev. Dispersion relation for acoustic waves in an anisotropic elastic half-space. Akustičeskij Žurnal, 35(5):922-928, 1989.

[67] T.C.T. Ting. Anisotropic Elasticity: Theory and Applications. Number 45. Oxford University Press on Demand, 1996.

[68] T.C.T. Ting. An explicit secular equation for surface waves in an elastic material of general anisotropy. Q. J. Mech. Appl. Math., 55(2):297-311, 2002.

[69] T.C.T. Ting. Secular equations for Rayleigh and Stoneley waves in exponentially graded elastic materials of general anisotropy under the influence of gravity. J. Elasticity, 105:331-347, 2011.

[70] T.C.T. Ting. Surface waves in an exponentially graded, general anisotropic elastic material under the influence of gravity. Wave Motion, 48:335-344, 2011.

[71] P.C. Vinh and P.G. Malischewsky. An approach for obtaining approximate formulas for the Rayleigh wave velocity. Wave Motion, 44:549-562, 2007.

[72] P.C. Vinh and P.G. Malischewsky. Improved approximations of the Rayleigh wave velocity. J. Thermoplast. Compos. Mater., 21:337-352, 2008.

[73] P.C. Vinh and R.W. Ogden. On formulas for the Rayleigh wave speed. Wave Motion, 39:191-197, 2004. 


\section{A An overview of the proofs from Fu and Mielke in our notation}

In this section, we will carry out a matrix algebraic analysis of the Riccati equation (5.5) of the linear Cosserat model to show that the properties listed in the previous section hold true for Cosserat isotropic materials, too. The content of this Appendix is almost entirely based on the results presented by Fu and Mielke in [25, 24] and we do not claim any merit in finding (and proving) them. The only aim of this appendix is to follow step by step the results from [25, 24] in order see that they can be applied in the framework of the linear isotropic Cosserat model, too.

Proposition A.1. (Exactly as from Fu and Mielke's paper) The matrix problem

$$
\mathcal{T} \mathcal{E}^{2}-\mathrm{i}\left(\mathcal{R}+\mathcal{R}^{T}\right) E-\mathcal{Q}+k^{2} v^{2} \mathbb{1}=0, \quad \operatorname{Respec} \mathcal{E}>0
$$

where $\operatorname{Respec} \mathcal{E}$ means the real part of spectra of $\mathcal{E}$ and $\mathcal{T}, \mathcal{T}, \mathcal{R}$ are defined in (3.7), has a unique solution for $\mathcal{E}$

Proof. Let $\mathcal{E}$ be the solution of (A.1). Let $r$ be the eigenvalue of $\mathcal{E}$ and $a$ be the associated eigenvector (so that $\mathcal{E} a=r a$ ). It follows from (A.1) that $r$ and $a$ must satisfy the eigenvalue problem

$$
\left\{r^{2} \mathcal{T}-\mathrm{i} r\left(\mathcal{R}+\mathcal{R}^{T}\right)-\mathcal{Q}+k^{2} v^{2} \mathbb{1}\right\} a=0, \quad \operatorname{det}\left\{r^{2} \mathcal{T}-\mathrm{i} r\left(\boldsymbol{\mathcal { R }}+\boldsymbol{\mathcal { R }}^{T}\right)-\mathcal{Q}+k^{2} v^{2} \mathbb{1}\right\}=0 .
$$

In the explicit form, the characteristic equation (A.2) 2 takes the form

$$
r^{6}+P_{1} r^{4}+P_{2} r^{2}+P_{3}=0,
$$

where $P_{1}, P_{2}, P_{3}, P_{4}$ are given by (4.8). We observe that characteristic equation is a six degree polynomial with real coefficients. If $r$ is the root of the characteristic equation then so is $-\bar{r}$. Thus, we conclude that the characteristic equation has three roots with positive real parts. Collecting the corresponding eigenspaces defines $\mathcal{E}$ uniquely.

Theorem A.2. (Exactly as from Fu and Mielke's paper) If $\mathcal{E}$ solves (A.1), then $\mathcal{M}$ obtained from (5.3) ${ }_{2}$ is Hermitian.

Proof. By taking transpose and complex conjugate of $(5.5)_{1}$ we obtain

$$
\left(\mathcal{M}^{T}+\mathrm{i} \mathcal{R}\right) \mathcal{T}^{-1}\left(\mathcal{M}-\mathrm{i} \mathcal{R}^{T}\right)-\mathcal{Q}^{T}+k^{2} v^{2} \mathbb{1}=0 \quad \Leftrightarrow \quad\left(\overline{\mathcal{M}}^{T}-\mathrm{i} \mathcal{R}\right) \mathcal{T}^{-1}\left(\overline{\mathcal{M}}+\mathrm{i} \mathcal{R}^{T}\right)-\mathcal{Q}^{T}+k^{2} v^{2} \mathbb{1}=0 .
$$

On subtracting $(\mathrm{A} .4)_{1}$ from $(\mathrm{A} .4)_{2}$, we get

$$
\left(\mathcal{M}-\overline{\mathcal{M}}^{T}\right) \mathcal{E}+\overline{\mathcal{E}}^{T}\left(\mathcal{M}-\overline{\mathcal{M}}^{T}\right)=0,
$$

which has the form of a Liaponov matrix equation

$$
\mathbf{X} \mathcal{E}+\overline{\mathcal{E}}^{T} \mathbf{X}=\mathbf{B} .
$$

The equation (A.6) has a unique solution $\mathbf{X}$. For $\operatorname{Re} \operatorname{spec}(\mathcal{E})>0$ it is given [3] by

$$
\mathbf{X}=\int_{0}^{\infty} e^{-t \overline{\boldsymbol{\varepsilon}}^{T}} \mathbf{B} e^{-t \boldsymbol{\varepsilon}} d t
$$

Since we have $\mathbf{B}=0$ the unique solution of (A.6) is $\mathbf{X}=0$ and hence $\mathcal{M}=\overline{\mathcal{M}}^{T}$.

Remark A.3. Every Hermitian matrix has real eigenvalues. The determinant of Hermitian matrix is a real number. A Hermitian matrix is said to be postive positive if it has positive eigenvalues.

In the proof of the following theorem, there is only a small difference compared to the proof given by Fu and Mielke [24].

Theorem A.4. (Exactly as from Fu and Mielke's paper) Let $\mathcal{M}$ and $\mathcal{E}$ be the same as in Theorem A.2. Then the matrix $\frac{d \mathcal{M}}{d v}$ is negative definite.

Proof. Differentiation (5.5) 1 w.r.t $v$ gives

$$
\begin{aligned}
\frac{d}{d v}(\mathcal{M}-\mathrm{i} \mathcal{R}) \mathcal{E}+(\mathcal{M}-\mathrm{i} \mathcal{R}) \frac{d}{d v} \mathcal{E}-\frac{d}{d v} \mathcal{Q}+\frac{d}{d v}\left(k^{2} v^{2} \mathbb{1}\right) & =0 \\
\frac{d}{d v} \mathcal{M} \mathcal{E}+(\mathcal{M}-\mathrm{i} \mathcal{R}) \frac{d}{d v} \mathcal{E} & =-2 v k^{2} \mathbb{1}, \\
\frac{d}{d v} \mathcal{M} \mathcal{E}+(\mathcal{M}-\mathrm{i} \mathcal{R}) \frac{d}{d v}\left\{\mathcal{T}^{-1}\left(\mathcal{M}+\mathrm{i} \mathcal{R}^{T}\right)\right\} & =-2 v k^{2} \mathbb{1}, \\
\frac{d}{d v} \mathcal{M} \mathcal{E}+(\mathcal{M}-\mathrm{i} \mathcal{R}) \mathcal{T}^{-1} \frac{d}{d v} \mathcal{M} & =-2 v k^{2} \mathbb{1}, \\
\frac{d}{d v} \mathcal{M} \mathcal{E}+\left(\overline{\mathcal{M}}^{T}-\mathrm{i} \mathcal{R}\right) \mathcal{T}^{-1} \frac{d}{d v} \mathcal{M} & =-2 v k^{2} \mathbb{1}, \quad \mathcal{M}=\overline{\mathcal{M}}^{T}
\end{aligned}
$$

Finally, we obtain

$$
\frac{d}{d v} \mathcal{M} \mathcal{E}+\overline{\mathcal{E}}^{T} \frac{d}{d v} \mathcal{M}=-2 k^{2} v \mathbb{1} .
$$

Equation (A.9) has a unique solution for $\mathcal{M}$ given by

$$
\frac{d}{d v} \mathcal{M}=\int_{0}^{\infty} e^{-t \overline{\boldsymbol{\varepsilon}}^{T}}\left(-2 \rho k^{2} v \mathbb{1}\right) e^{-t \boldsymbol{\mathcal { E }}} d t=-2 k^{2} v \int_{0}^{\infty} e^{-t \overline{\boldsymbol{\varepsilon}}^{T}} \mathbb{1} e^{-t \boldsymbol{\mathcal { E }}} d t .
$$


Thus, for arbitrary non-zero vector $\eta \in \mathbb{C}^{n}$ we have

$$
\begin{aligned}
\left\langle\bar{\eta}, \frac{d}{d v} \mathcal{M} \eta\right\rangle & =-2 k^{2} v\left\langle\bar{\eta}, \int_{0}^{\infty} e^{-t \overline{\mathcal{E}}^{T}} \mathbb{1} e^{-t \mathcal{E}} d t \eta\right\rangle_{\mathbb{C}^{n}}=-2 k^{2} v \int_{0}^{\infty}\left\langle\overline{e^{-t \mathcal{E}_{\eta}}}, e^{-t \boldsymbol{\varepsilon}} d t \eta\right\rangle_{\mathbb{C}^{n}}, \\
& =-2 k^{2} v \int_{0}^{\infty}\langle\bar{\zeta}(t), \zeta(t)\rangle_{\mathbb{C}^{n}} d t=-2 k^{2} v \int_{0}^{\infty} \underbrace{\|\zeta(t)\|^{2}}_{\geq a^{+}>0} d t, \quad \text { where } \quad \zeta(t)=e^{-t \boldsymbol{\mathcal { E }} \eta},
\end{aligned}
$$

Since $\zeta(0)=\eta \neq 0$ and $\zeta(t)$ is continuous at $t=0$ (so that $\zeta(t)$ is non-zero at least in a small but finite interval), we have $\left\langle\bar{\eta}, \frac{d}{d v} \mathcal{M} \eta\right\rangle<0$ and hence $\frac{d}{d v} \mathcal{M}$ is negative definite.

Theorem A.5. (Exactly as from Fu and Mielke's paper) The Hermitian matrix $\mathcal{M}_{\theta}$ satisfying (5.16) is independent of $\theta$.

Proof. Only in this proof, we denote by $f^{\prime}(\theta)=\frac{d f}{d \theta}(\theta)$. On differentiating $(5.14)_{1}$ w.r.t $\theta$ we get

$$
\mathcal{T}_{\theta}^{\prime}=2 \sin \theta \cos \theta(\widetilde{\mathcal{Q}}-\mathcal{T})-\cos ^{2} \theta\left(\mathcal{R}+\mathcal{R}^{T}\right)+\sin ^{2} \theta\left(\mathcal{R}+\mathcal{R}^{T}\right) .
$$

It is obvious to see

$$
\mathcal{R}_{\theta}^{T}=\cos ^{2} \theta \mathcal{R}^{T}-\sin ^{2} \theta \mathcal{R}+\sin \theta \cos \theta(\mathcal{T}-\widetilde{\mathcal{Q}})
$$

and

$$
\begin{gathered}
-\boldsymbol{\mathcal { R }}_{\theta}-\boldsymbol{\mathcal { R }}_{\theta}^{T}=2 \sin \theta \cos \theta(\widetilde{\mathcal{Q}}-\mathcal{T})-\cos ^{2} \theta\left(\mathcal{R}+\mathcal{R}^{T}\right)+\sin ^{2} \theta\left(\mathcal{R}+\mathcal{R}^{T}\right), \\
\mathcal{T}_{\theta}^{\prime}=-\mathcal{R}_{\theta}-\mathcal{R}_{\theta}^{T}
\end{gathered}
$$

Similarly,

$$
\mathcal{R}_{\theta}^{\prime}=-2 \cos \theta \sin \theta\left(\mathcal{R}+\mathcal{R}^{T}\right)+\left(\cos ^{2} \theta-\sin ^{2} \theta\right)(\mathcal{T}-\widetilde{\mathcal{Q}})
$$

and we see that

$$
\mathcal{T}_{\theta}-\mathcal{Q}_{\theta}=\left(\cos ^{2} \theta-\sin ^{2} \theta\right)(\mathcal{T}-\widetilde{\mathcal{Q}})-2 \sin \theta \cos \theta\left(\mathcal{R}+\mathcal{R}^{T}\right)
$$

Therefore

$$
\mathcal{R}_{\theta}^{\prime}=\mathcal{T}_{\theta}-\mathcal{Q}_{\theta}
$$

and

$$
\mathcal{Q}_{\theta}^{\prime}=2 \sin \theta \cos \theta(\mathcal{T}-\mathcal{Q})+\cos ^{2} \theta\left(\mathcal{R}+\mathcal{R}^{T}\right)-\sin ^{2} \theta\left(\mathcal{R}+\mathcal{R}^{T}\right)
$$

It is also easy to see

$$
\mathcal{Q}_{\theta}^{\prime}=\boldsymbol{\mathcal { R }}_{\theta}+\boldsymbol{\mathcal { R }}_{\theta}^{T},
$$

and after differentiating $\mathcal{T}_{\theta} \mathcal{T}_{\theta}^{-1}=\mathbb{1}$, we also get

$$
\left(\mathcal{T}_{\theta}^{-1}\right)^{\prime}=-\mathcal{T}_{\theta}^{-1} \mathcal{T}_{\theta}^{\prime} \mathcal{T}_{\theta}^{-1}
$$

On differentiating $(5.5)_{1}$ w.r.t $\theta$ we get

$$
\begin{array}{r}
\left(\mathcal{M}_{\theta}-\mathrm{i} \mathcal{R}_{\theta}\right)^{\prime} \mathcal{E}_{\theta}+\left(\mathcal{M}_{\theta}-\mathrm{i} \mathcal{R}_{\theta}\right) \mathcal{E}_{\theta}^{\prime}-\mathcal{M}_{\theta}^{\prime}=0, \\
\left(\mathcal{M}_{\theta}^{\prime}-\mathrm{i} \mathcal{R}_{\theta}^{\prime}\right) \mathcal{E}_{\theta}+\left(\mathcal{M}_{\theta}-\mathrm{i} \mathcal{R}_{\theta}\right) \mathcal{T}_{\theta}^{-1}\left\{\left(\mathcal{M}_{\theta}^{\prime}+\mathrm{i}\left(\mathcal{R}_{\theta}^{T}\right)^{\prime}\right)-\mathcal{T}_{\theta}^{\prime} \mathcal{T}_{\theta}^{-1}\left(\mathcal{M}_{\theta}+\mathrm{i} \mathcal{R}_{\theta}^{T}\right)\right\}-\mathcal{Q}_{\theta}^{\prime}=0, \\
\left(\mathcal{M}_{\theta}^{\prime}-\mathrm{i} \mathcal{R}_{\theta}^{\prime}\right) \mathcal{E}_{\theta}+\overline{\mathcal{E}}_{\theta}^{T}\left(\mathcal{M}_{\theta}^{\prime}+\mathrm{i}\left(\mathcal{R}_{\theta}^{T}\right)^{\prime}\right)-\overline{\mathcal{E}}_{\theta}^{T} \mathcal{T}_{\theta}^{\prime} \mathcal{E}_{\theta}-\mathcal{Q}_{\theta}^{\prime}=0, \\
\mathcal{M}_{\theta}^{\prime} \mathcal{E}_{\theta}+\overline{\mathcal{E}}_{\theta}^{T} \mathcal{M}_{\theta}^{\prime}-\mathrm{i} \mathcal{R}_{\theta}^{\prime} \mathcal{E}_{\theta}+\mathrm{i} \overline{\mathcal{E}}_{\theta}^{T}\left(\mathcal{R}_{\theta}^{T}\right)^{\prime}-\overline{\mathcal{E}}_{\theta}^{T} \mathcal{T}_{\theta}^{\prime} \mathcal{E}_{\theta}-\mathcal{Q}_{\theta}^{\prime}=0,
\end{array}
$$

and using (A.13),(A.14) and (A.15) in (A.17) 6 , yields

$$
\begin{array}{r}
\mathcal{M}_{\theta}^{\prime} \mathcal{E}_{\theta}+\overline{\mathcal{E}}_{\theta}^{T} \mathcal{M}_{\theta}^{\prime}-\mathrm{i}\left(\mathcal{R}_{\theta}\right)^{\prime} \mathcal{E}_{\theta}+\mathrm{i} \overline{\mathcal{E}}_{\theta}^{T}\left(\boldsymbol{\mathcal { R }}_{\theta}\right)^{\prime}-\overline{\mathcal{E}}_{\theta}^{T} \mathcal{T}_{\theta}^{\prime} \mathcal{E}_{\theta}-\mathcal{Q}_{\theta}^{\prime}=0 \\
\mathcal{M}_{\theta}^{\prime} \mathcal{E}_{\theta}+\overline{\mathcal{E}}_{\theta}^{T} \mathcal{M}_{\theta}^{\prime}-\mathrm{i}\left(\mathcal{T}_{\theta}-\mathcal{Q}_{\theta}\right) \mathcal{E}_{\theta}+\mathrm{i} \overline{\mathcal{E}}_{\theta}^{T}\left(\mathcal{T}_{\theta}-\mathcal{Q}_{\theta}\right)+\mathcal{E}_{\theta}^{T}\left(\mathcal{R}_{\theta}+\mathcal{R}_{\theta}^{T}\right) \mathcal{E}_{\theta}-\left(\mathcal{R}_{\theta}+\mathcal{R}_{\theta}^{T}\right)=0
\end{array}
$$

On replacing first $\mathcal{Q}_{\theta}$ in (A.18) $)_{2}$ by $\mathcal{E}_{\theta}^{T}\left(\mathcal{M}_{\theta}+\mathrm{i} \boldsymbol{\mathcal { R }}_{\theta}^{T}\right)$ and the second $\boldsymbol{Q}_{\theta}$ by $\left(\boldsymbol{M}_{\theta}-\mathrm{i} \boldsymbol{\mathcal { R }}_{\theta}\right) \mathcal{E}_{\theta}$, see (5.18), we deduce

$$
\begin{aligned}
& \mathcal{M}_{\theta}^{\prime} \mathcal{E}_{\theta}+\mathcal{E}_{\theta}^{T} \mathcal{M}_{\theta}^{\prime}-\mathrm{i}\left[\left\{\mathcal{T}_{\theta}-\mathcal{E}_{\theta}^{T}\left(\mathcal{M}_{\theta}+\mathrm{i} \boldsymbol{\mathcal { R }}_{\theta}^{T}\right)\right\} \mathcal{E}_{\theta}+\mathrm{i} \mathcal{E}_{\theta}^{T}\left\{\mathcal{T}_{\theta}-\left(\mathcal{M}_{\theta}-\mathrm{i} \boldsymbol{R}_{\theta}\right) \mathcal{E}_{\theta}\right\}\right]+\mathcal{E}_{\theta}^{T}\left(\boldsymbol{\mathcal { R }}_{\theta}+\boldsymbol{\mathcal { R }}_{\theta}^{T}\right) \mathcal{E}_{\theta}-\left(\boldsymbol{\mathcal { R }}_{\theta}+\boldsymbol{\mathcal { R }}_{\theta}^{T}\right)=0 \\
& \mathcal{M}_{\theta}^{\prime} \mathcal{E}_{\theta}+\mathcal{E}_{\theta}^{T} \mathcal{M}_{\theta}^{\prime}-\mathrm{i} \mathcal{T}_{\theta} \mathcal{E}_{\theta}+\mathrm{i} \mathcal{E}_{\theta}^{T} \mathcal{T}_{\theta}-\mathcal{E}_{\theta}^{T} \boldsymbol{\mathcal { R }}_{\theta}^{T} \mathcal{E}_{\theta}-\mathcal{E}_{\theta}^{T} \boldsymbol{\mathcal { R }}_{\theta} \mathcal{E}_{\theta}+\mathcal{E}_{\theta}^{T}\left(\boldsymbol{R}_{\theta}+\boldsymbol{\mathcal { R }}_{\theta}^{T}\right) \mathcal{E}_{\theta}-\left(\boldsymbol{R}_{\theta}+\mathcal{R}_{\theta}^{T}\right)=0 \\
& \mathcal{M}_{\theta}^{\prime} \mathcal{E}_{\theta}+\mathcal{E}_{\theta}^{T} \mathcal{M}_{\theta}^{\prime}-\mathrm{i} \mathcal{T}_{\theta} \mathcal{E}_{\theta}+\mathrm{i} \mathcal{E}_{\theta}^{T} \mathcal{T}_{\theta}-\left(\mathcal{R}_{\theta}+\mathcal{R}_{\theta}^{T}\right)=0 \\
& \mathcal{M}_{\theta}^{\prime} \mathcal{E}_{\theta}+\mathcal{E}_{\theta}^{T} \mathcal{M}_{\theta}^{\prime}-\mathrm{i} \mathcal{T}_{\theta}\left\{\mathcal{T}_{\theta}^{-1}\left(\mathcal{M}_{\theta}+\mathrm{i} \boldsymbol{\mathcal { R }}_{\theta}^{T}\right)\right\}-\left(\boldsymbol{\mathcal { R }}_{\theta}+\boldsymbol{\mathcal { R }}_{\theta}^{T}\right)+\mathrm{i}\left\{\left(\mathcal{M}_{\theta}-\mathrm{i} \boldsymbol{\mathcal { R }}_{\theta}\right) \boldsymbol{\mathcal { T }}_{\theta}^{-1}\right\} \boldsymbol{\mathcal { T }}_{\theta}=0 \\
& \mathcal{M}_{\theta}^{\prime} \mathcal{E}_{\theta}+\mathcal{E}_{\theta}^{T} \mathcal{M}_{\theta}^{\prime}-\mathrm{i} \mathcal{M}_{\theta}+\mathrm{i} \mathcal{M}_{\theta}+\left(\mathcal{R}_{\theta}+\mathcal{R}_{\theta}^{T}\right)-\left(\mathcal{R}_{\theta}+\mathcal{R}_{\theta}^{T}\right)=0
\end{aligned}
$$

finaly we obtain,

$$
\mathcal{M}_{\theta}^{\prime} \mathcal{E}_{\theta}+\mathcal{E}_{\theta}^{T} \mathcal{M}_{\theta}^{\prime}=0
$$

which is another homogeneous Liaponov matrix equation. It then follows that $\boldsymbol{\mathcal { M }}_{\theta}^{\prime}=0$, (see (A.6)) so $\boldsymbol{M}_{\theta}$ is independent of $\theta$. 
We have observed that $\mathcal{E}_{\theta}$ reduces to $\mathcal{E}$ defined in Theorem 2.1 when $\theta=0$, we have $\mathcal{M}_{\theta} \equiv \mathcal{M}$, where $\mathcal{M}$ is the corresponding $\mathcal{M}$ given in Theorem (2.1). Thus

$$
\mathcal{E}_{\theta}=\mathcal{T}_{\theta}^{-1}\left(\mathcal{M}+\mathrm{i} \mathcal{R}_{\theta}^{T}\right)
$$

On differentiating (A.21) with respect to $\theta$, we get

$$
\mathcal{E}_{\theta}^{\prime}=\left(\mathcal{T}_{\theta}^{-1}\right)^{\prime}\left(\mathcal{M}+\mathrm{i} \boldsymbol{\mathcal { R }}_{\theta}^{T}\right)+\mathcal{T}_{\theta}^{-1}\left(\mathcal{M}+\mathrm{i} \boldsymbol{\mathcal { R }}_{\theta}^{T}\right)^{\prime}=\left(\mathcal{T}_{\theta}^{-1}\right)^{\prime}\left(\mathcal{M}+\mathrm{i} \boldsymbol{\mathcal { R }}_{\theta}^{T}\right)+\mathcal{T}_{\theta}^{-1}\left(\mathrm{i} \boldsymbol{\mathcal { R }}_{\theta}^{T}\right)^{\prime}
$$

making use of (A.16), we obtain

$$
\mathcal{E}_{\theta}^{\prime}=-\mathcal{T}_{\theta}^{-1} \mathcal{T}_{\theta}^{\prime} \mathcal{T}_{\theta}^{-1}\left(\mathcal{M}+\mathrm{i} \mathcal{R}_{\theta}^{T}\right)+\mathrm{i} \mathcal{T}_{\theta}^{-1}\left(\mathcal{T}_{\theta}-\mathcal{Q}_{\theta}\right)=\mathcal{T}_{\theta}^{-1}\left(\mathcal{R}_{\theta}+\mathcal{R}_{\theta}^{T}\right)+\mathrm{i}\left(\mathbb{1}-\mathcal{T}_{\theta}^{-1} \mathcal{Q}_{\theta}\right)
$$

From (A.21) it is easy to see

$$
\mathcal{R}_{\theta}^{T}=\mathrm{i} \mathcal{M}-\mathrm{i} \mathcal{T}_{\theta} \mathcal{E}_{\theta}
$$

which after substituting (A.24) in (A.23) $)_{2}$, leads us to

$$
\begin{aligned}
\mathcal{E}_{\theta}^{\prime} & \left.=\mathcal{T}_{\theta}^{-1}\left\{\mathcal{R}_{\theta}+\mathrm{i}\left(\mathcal{M}-\mathcal{T}_{\theta}\right) \mathcal{E}_{\theta}\right\} \mathcal{E}_{\theta}+\mathrm{i}\left(\mathbb{1}-\mathcal{T}_{\theta}^{-1} \mathcal{Q}_{\theta}\right)=\mathcal{T}_{\theta}^{-1}\left\{\mathrm{i}\left(\mathcal{M}-\mathrm{i} \mathcal{R}_{\theta}\right)-\mathrm{i} \mathcal{T}_{\theta}\right) \mathcal{E}_{\theta}\right\} \mathcal{E}_{\theta}+\mathrm{i}\left(\mathbb{1}-\mathcal{T}_{\theta}^{-1} \mathcal{Q}_{\theta}\right) \\
& =\mathcal{T}_{\theta}^{-1}\left\{\mathrm{i} \mathcal{Q}-\mathrm{i} \mathcal{T}_{\theta} \mathcal{E}_{\theta}\right\} \mathcal{E}_{\theta}+\mathrm{i}\left(\mathbb{1}-\mathcal{T}_{\theta}^{-1} \mathcal{Q}_{\theta}\right)=\underline{\mathrm{i}} \mathcal{T}_{\theta}^{-1} \mathcal{Q}-\mathrm{i} \mathcal{E}_{\theta}^{2}+\mathbb{1} \mathrm{i}-\mathrm{i} \mathcal{T}_{\theta}^{-1} \mathcal{Q}_{\theta}=\mathbb{1}-\mathrm{i} \mathcal{E}_{\theta}^{2}
\end{aligned}
$$

After integrating this matrix differential equation subject to the condition $\mathcal{E}_{0}=\mathcal{E}$, we obtain

Proposition A.6. (Exactly as from Fu and Mielke's paper) We have

$$
\mathcal{E}_{\theta}=(\cos \theta \mathbb{1}+\mathrm{i} \sin \theta \mathcal{E})^{-1}(\cos \theta \mathcal{E}+\mathrm{i} \sin \theta \mathbb{1}) .
$$

Proof. Since, $\mathbf{J}(\theta) \mathbf{J}^{-1}(\theta)=\mathbb{1}$ after differentiating, it can be observed that for any matrix $\mathbf{J}(\theta)$, we have

$$
\left(\mathbf{J}^{-1}(\theta)\right)^{\prime}=-\mathbf{J}^{-1}(\theta) \mathbf{J}^{\prime}(\theta) \mathbf{J}^{-1}(\theta),
$$

it then follows that

$$
\left.\left(\mathbf{J}^{-1}(\theta) \mathbf{J}^{\prime}(\theta)\right)^{\prime}=-\left(\mathbf{J}^{-1}(\theta) \mathbf{J}^{\prime}(\theta)\right)^{2}+\mathbf{J}^{-1}(\theta) \mathbf{J}^{\prime \prime} \theta\right),
$$

or equivalently

$$
\left.\left(-\mathrm{i} \mathbf{J}^{-1}(\theta) \mathbf{J}^{\prime}(\theta)\right)^{\prime}=-\mathrm{i}\left(-\mathrm{i} \mathbf{J}^{-1}(\theta) \mathbf{J}^{\prime}(\theta)\right)^{2}-\mathrm{i} \mathbf{J}^{-1}(\theta) \mathbf{J}^{\prime \prime} \theta\right) .
$$

This suggests the transformation

$$
\mathcal{E}_{\theta}=-\mathrm{i} \mathbf{J}^{-1}(\theta) \mathbf{J}^{\prime}(\theta)
$$

since on substituting (A.30) in (A.25), we arrive at

$$
\left.\mathbf{J}^{\prime \prime} \theta\right)+\mathbf{J}(\theta)=0,
$$

the general solution of (A.31) is

$$
\mathbf{J}(\theta)=\cos \theta \mathbf{c}_{l}+\mathrm{i} \sin \theta \mathbf{c}_{t},
$$

where, $\mathbf{c}_{l}$ and $\mathbf{c}_{t}$ are constant matrices. From the condition $\mathcal{E}_{0}=\mathcal{E}$ we obtain $\mathcal{E}=-\mathrm{i} \mathbf{c}_{l} \mathbf{c}_{t}$. Thus, $\mathbf{J}(\theta)=\mathbf{c}_{l}(\cos \theta \mathbb{1}+\mathrm{i} \sin \theta \mathcal{E})$ and

$$
\mathcal{E}_{\theta}=-\mathrm{i} \mathbf{J}^{-1}(\theta) \mathbf{J}(\theta)=(\cos \theta \mathbb{1}+\mathrm{i} \sin \theta \mathcal{E})^{-1}(\cos \theta \mathcal{E}+\mathrm{i} \sin \theta \mathbb{1})
$$

and the proof is complete.

If $\lambda$ is an eigenvalue of $\mathcal{E}$. then by (A.1) the corresponding eigenvalues of $\mathcal{E}_{\theta}$ is

$$
\lambda(\theta)=\Phi\left(\lambda_{\mathrm{e}}, \theta\right), \quad \text { where } \quad \Phi(\lambda, \theta)=\frac{\lambda \cos \theta+\mathrm{i} \sin \theta}{\cos \theta+\mathrm{i} \lambda \sin \theta}=\mathrm{i} \frac{d}{d \theta} \ln (\cos \theta+\mathrm{i} \lambda \sin \theta) .
$$

The real part of $\lambda(\theta)$ is indeed positive. It then follows

$$
\int_{0}^{\pi} \lambda(\theta) d \theta=-\mathrm{i} \int_{0}^{\pi} \frac{d}{d \theta} \ln (\cos \theta+\mathrm{i} \lambda \sin \theta) d \theta=-\left.\mathrm{i} \ln (\cos \theta+\mathrm{i} \lambda \sin \theta)\right|_{0} ^{\pi}=\pi .
$$

So this leads to

Proposition A.7. (Exactly as from Fu and Mielke's paper) We have

$$
\int_{0}^{\pi} \mathcal{E}_{\theta} d \theta=\pi \mathbb{1}
$$

Proof. We use the spectral calculus of matrices. By choosing any closed curve $\Gamma$ in the complex plane surrounding all the eigenvectors of $\mathcal{E}$ with positive real parts and lying in the open half plane $\operatorname{Re} \lambda>0$. Then by Proposition 2.1 we have

$$
\begin{aligned}
\int_{0}^{\pi} \mathcal{E}_{\theta} d \theta & =\int_{0}^{\pi} \frac{1}{2 \pi \mathrm{i}} \oint_{\Gamma} \Phi(\lambda, \theta)(\lambda \mathbb{1}-\mathcal{E})^{-1} d \lambda d \theta=\frac{1}{2 \pi \mathrm{i}} \oint_{\Gamma} \int_{0}^{\pi} \Phi\left(\lambda_{\mathrm{e}}, \theta\right) d \theta(\lambda \mathbb{1}-\mathcal{E})^{-1} d \lambda_{\mathrm{e}} \\
& =\frac{1}{2 \pi \mathrm{i}} \oint_{\Gamma}(\lambda \mathbb{1}-\mathcal{E})^{-1} \pi d \lambda_{\mathrm{e}}
\end{aligned}
$$

The conclusion of the proposition follows then since $\int_{0}^{\pi} \lambda_{\mathrm{e}}(\theta) d \theta=\pi$. 
On integrating (A.21), we get

$$
\begin{aligned}
\int_{0}^{\pi} \mathcal{E}_{\theta} d \theta=\int_{0}^{\pi} \mathcal{T}_{\theta}^{-1}\left(\mathcal{M}+\mathrm{i} \mathcal{R}_{\theta}^{T}\right) d \theta & \Leftrightarrow \int_{0}^{\pi} \mathcal{T}_{\theta}^{-1} \mathcal{M} d \theta=\int_{0}^{\pi} \mathcal{E}_{\theta} d \theta-\mathrm{i} \int_{0}^{\pi} \mathcal{T}_{\theta}^{-1} \mathcal{R}_{\theta}^{T} d \theta \\
& \Leftrightarrow \quad\left(\int_{0}^{\pi} \mathcal{T}_{\theta}^{-1} d \theta\right) \mathcal{M}=\pi \mathbb{1}-\mathrm{i} \int_{0}^{\pi} \mathcal{T}_{\theta}^{-1} \mathcal{R}_{\theta}^{T} d \theta
\end{aligned}
$$

Finally we obtain

Theorem A.8. (Exactly as from Fu and Mielke's paper) The unique solution of the algebraic Riccati equation (5.5) that satisfies $\operatorname{Respec}\left(\mathcal{T}^{-1}\left(\mathcal{M}+\mathrm{i} \mathcal{R}^{T}\right)\right)>0$ is given explicitly by

$$
\mathcal{M}=\left(\int_{0}^{\pi} \mathcal{T}_{\theta}^{-1} d \theta\right)^{-1}\left(\pi \mathbb{1}-\mathrm{i} \int_{0}^{\pi} \mathcal{T}_{\theta}^{-1} \boldsymbol{\mathcal { R }}_{\theta}^{T} d \theta\right)
$$

Proof. We multiply the relation (A.36) by $\left(\int_{0}^{\pi} \mathcal{T}_{\theta}^{-1} d \theta\right)^{-1}$. 